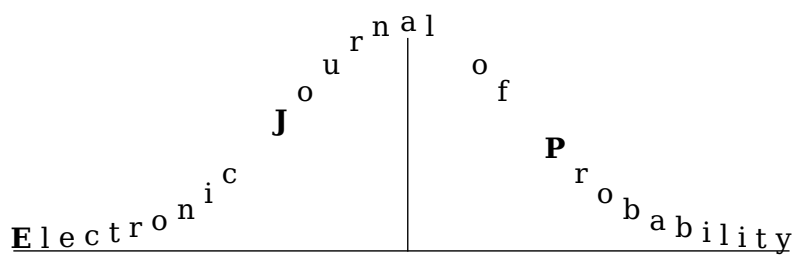

Electron. J. Probab. 27 (2022), article no. 34, 1-56.

ISSN: 1083-6489 https://doi.org/10.1214/22-EJP754

\title{
Tangent fields, intrinsic stationarity, and self similarity*
}

\author{
Jinqi Shen ${ }^{\dagger} \quad$ Stilian Stoev $^{\dagger} \quad$ Tailen Hsing $^{\dagger}$
}

Dedicated to the Memory of Mark Marvin Meerschaert (1955-2020)

\begin{abstract}
This paper studies the local structure of continuous random fields on $\mathbb{R}^{d}$ taking values in a complete separable linear metric space $\mathbb{V}$. Extending seminal work of Falconer, we show that the generalized $(1+k)$-th order increment tangent fields are self-similar and almost everywhere intrinsically stationary in the sense of Matheron. These results motivate the further study of the structure of $\mathbb{V}$-valued intrinsic random functions of order $k\left(\operatorname{IRF}_{k}, k=0,1, \ldots\right)$. To this end, we focus on the special case where $\mathbb{V}$ is a Hilbert space. Building on the work of Sasvari and Berschneider, we establish the spectral characterization of all second order $\mathbb{V}$-valued $\mathrm{IRF}_{k}$ 's, extending the classical Matheron theory. Using these results, we further characterize the class of Gaussian, operator self-similar $\mathbb{V}$-valued $\mathrm{IRF}_{k}$ 's, generalizing results of Dobrushin and Didier, Meerschaert and Pipiras, among others. These processes are the Hilbert-spacevalued versions of the general $k$-th order operator fractional Brownian fields and are characterized by their self-similarity operator exponent as well as a finite trace class operator valued spectral measure. We conclude with several examples motivating future applications to probability and statistics.
\end{abstract}

Keywords: tangent field; $\mathrm{IRF}_{k}$; operator self-similarity; spectral theory; functional data analysis. MSC2020 subject classifications: Primary 60G10; 60G12; 60G18; 60G22, Secondary 62R10; $62 \mathrm{H} 11$.

Submitted to EJP on January 5, 2021, final version accepted on February 6, 2022.

Supersedes arXiv:2010.14715v3.

\section{Introduction}

The tangent process of a random field is the stochastic process obtained in the limit of the suitably normalized increments of the random field at a fixed location. A pair of

\footnotetext{
*SS and TH were partially supported by the NSF Grant DMS-1916226 The Argo Data and Functional Spatial Processes.

${ }^{\dagger}$ Department of Statistics, University of Michigan, Ann Arbor, United States of America.

E-mail: jqshen@umich.edu, sstoev@umich.edu, thsing@umich.edu
} 
papers, Falconer $(2002,2003)$, discovered a remarkable property about the structure of the tangent process. Briefly speaking, Falconer proved that the tangent processes must be self-similar and have stationary increments (a.e.). The self-similarity of the tangent field is not surprising, which is a consequence of scaling. It is akin to what is shown in many limit theorems in the literature, such as those in the seminal work of Lamperti (cf. Lamperti, 1962); see also Davydov and Paulauskas (2017) and a host of results on (univariate and multivariate) regular variation in Gnedenko (1943); Meerschaert (1984); Hult and Lindskog (2006). The property of stationary increments for the tangent process, however, is less expected. The proof of this property in Falconer's works involves a remarkable Lebesgue-density argument and ideas from geometric measure theory (cf. Falconer, 1986; Preiss, 1987).

The starting point of our paper is extending Falconer's results in two directions. Firstly, we consider generalized tangent processes obtained by taking local $(1+k)$-th order increments, $k \geq 0$. This is necessary if one wants to study the local behavior of many models arising in spatial statistics. Secondly, we consider random fields taking values in a linear complete separable metric space $\mathbb{V}$ such as but not limited to a separable Banach space. The resulting limit processes, will be referred to as $k$-th order tangent processes. In Falconer $(2002,2003), k=0$ and $\mathbb{V}=\mathbb{R}$. The self-similarity property continues to hold for $k$-th order tangent processes, where self-similarity is in the sense of a general class of scaling actions, including operator scaling (cf. Meerschaert and Scheffler, 2001), that commensurate with the generality of the state space $\mathbb{V}$. To establish the generalized stationary-increment property, we introduce a new proof strategy based on the Lusin and Egorov theorems as well as some core ideas in Falconer (2002, 2003).

Interestingly, the stationarity of the higher-order increments of the $k$-th order tangent processes is related to the notion of intrinsic random functions of order $k\left(\operatorname{IRF}_{k}\right)$ introduced by Matheron (1973). In the special case of real-valued processes $(\mathbb{V}=\mathbb{R})$, the classic results of Matheron as well as Gel'fand and Vilenkin (1964) lead to a concrete formula for all possible covariance structures of the $k$-th order tangent fields. It involves the local self-similarity exponent $H \in(0, k+1]$ and the local spectral measure $\sigma$. Such results have been established by Dobrushin (1979) in the setting where the paths of the stochastic processes are generalized functions, i.e., random elements in $\mathcal{S}^{\prime}\left(\mathbb{R}^{d}\right)$ - the topological dual of the Schwartz space $\mathcal{S}\left(\mathbb{R}^{d}\right)$. Our study, motivated by applications to spatial statistics and functional data analysis, considers random fields taking values in a separable Hilbert space $\mathbb{V}$. We follow the approach of Matheron rather than Dobrushin and realize the notion of a higher-order increment by integrating the process against signed measures with finite supports.

To this end, in Section 4, we develop an extension of Matheron's theory to the case of processes taking values in a separable Hilbert space $\mathbb{V}$. Our theoretical development for Hilbert-space-valued $\mathrm{IRF}_{k}$ 's is of independent interest and builds on a large body of existing although somewhat scattered work. With no intention to provide a complete list, we refer to Bochner (1948) and Khintchine (1934) for Bochner's theorem and Neeb (1998) for extensions to general spaces; Cramér (1942) for the spectral representation of stationary random fields; Matheron (1973), Sasvári (2009) for the existence of general covariance of $\operatorname{IRF}_{k}$ and its integral representation; Berschneider (2012) for the integral representation of $\mathrm{IRF}_{k}$ in an abstract space. A more comprehensive summary on this line of literature can be found in Berschneider and Sasvári (2018). Our proofs in this regard are contained in the extended version of this paper (Shen et al., 2020), which aims to be self-contained and only uses arguments that are common in probability and statistics.

The developed theory is then utilized in Section 5 to characterize the covariance structure of self-similar intrinsic random functions taking values in a separable Hilbert 
Tangent fields, intrinsic stationarity, and self similarity

space. General linear operator scaling actions are considered as well as the classic multiplication by a scalar. In particular, our results provide characterizations of Gaussian operator self-similar $\mathrm{IRF}_{k}$ 's, which can be viewed as infinite-dimensional versions of the $k$-th order fractional Brownian fields. The 0-th order operator fractional Brownian fields taking values in $\mathbb{V}=\mathbb{R}^{m}$ have been the subject of active investigation and numerous applications (see e.g. Mason and Xiao, 2001; Amblard and Coeurjolly, 2011; Kechagias and Pipiras, 2015; Abry and Didier, 2018; Abry et al., 2019; Düker, 2020; Biermé and Lacaux, 2020, and the references therein). Most if not all of the existing work, however, focuses on random fields taking values in $\mathbb{R}^{m}$. In this paper, we provide a first comprehensive treatment of Hilbert space valued operator fractional Brownian fields and their higher order stationary increment counterparts - the Gaussian $\mathrm{IRF}_{k}{ }^{\text {'s. This }}$ leads to infinite-dimensional extensions of seminal results due to Didier and Pipiras (2011); Didier et al. (2017); Perrin et al. (2001) among others.

This paper also contributes to statistical research in several ways. Matheron's work on $\mathbb{R}$-valued $\mathrm{IRF}_{k}$ 's has had a substantial impact on the field of spatial statistics (see e.g. the monographs of Stein, 1999; Chilès and Delfiner, 2012). Our extension of the Matheron theory to the case of Hilbert-space-valued random fields provides novel tools and framework for spatially dependent functional data analysis - an active area in statistics (see, e.g., the monographs Ramsay and Silverman, 2005; Horváth and Kokoszka, 2012; Hsing and Eubank, 2015, and references therein). Unfortunately, the details of Matheron's theory have been elusive to the broader community. Our work and its extended version Shen et al. (2020) will be a useful resource for those who are interested in learning those details and their novel generalizations. The self-similar $\operatorname{IRF}_{k}$ is itself a flexible model for spatial statistics. The self-similarity exponent operator $\mathcal{H}$ and spectral measure $\sigma$, which now takes values in the space of positive trace-class operators on $\mathbb{V}$ characterize the covariance structure. The pair $(\mathcal{H}, \sigma)$ may be object of further modeling and inference in the context of in-fill asymptotics (cf. Stein, 1999). The tangent process connection also provides guidance for building flexible random field models with desired local properties.

The paper is organized as follows. Section 2 introduces a suitable topology on the path space and scaling actions needed to define and study higher-order tangent fields. In Section 3, we establish the main results on the structure of higher-order tangent fields, namely their self-similarity and almost everywhere intrinsic stationarity. Section 4 develops the spectral theory for second-order stationary and intrinsically stationary random fields taking values in a separable Hilbert space $\mathbb{V}$. This treatment unifies and extends results of Bochner, Cramér, Gelfand-Vilenkin, Matheron, Neeb, Sasvári, and Berschneider. The covariance structure of the self-similar $\mathbb{V}$-valued $\mathrm{IRF}_{k}$ is characterized in Section 5. General linear operator-scaling actions (Section 5.1) and the classic scalar scaling actions (Section 5.3) are studied. Open problems, examples and connections to the existing literature are presented in Section 5.2. Some technical proofs are relegated to the Appendix. Further background and details are given in Shen et al. (2020).

\section{Preliminaries}

This section develops some tools that will be useful for the study of tangent fields. We commence by defining some key spaces and operations.

For $d=1,2, \ldots$, let $\Lambda$ denote the collection of complex-valued measures on $\mathbb{R}^{d}$ supported on finitely many points, i.e.,

$$
\lambda(d u)=\sum_{i=1}^{n} c_{i} \delta_{t_{i}}(d u),
$$


where $n=1,2, \ldots, c_{i} \in \mathbb{C}, t_{i} \in \mathbb{R}^{d}$ for all $i$, and $\delta_{a}$ is the Dirac measure at $a$. Without loss of generality, we always assume the $t_{i}$ 's in the representation (2.1) to be distinct. In this paper we will be concerned with functions defined on $\Lambda$ or a subspace of $\Lambda$ and take values in some vector space $\mathbb{V}$. A special case of that is

$$
g(\lambda):=\int g d \lambda \equiv \sum_{i=1}^{n} c_{i} g\left(t_{i}\right)
$$

for any $g: \mathbb{R}^{d} \mapsto \mathbb{V}$.

For any $k \in \mathbb{N}:=\{0,1,2, \ldots\}$, a monomial of degree $k$ on $\mathbb{R}^{d}$ is any function of the form $u=\left(u_{1}, \ldots, u_{d}\right) \mapsto u_{1}^{j_{1}} \cdots u_{d}^{j_{d}}$, where $j_{1}, \ldots, j_{d}$ are non-negative integer powers such that $j_{1}+\cdots+j_{d}=k$. More generally, a polynomial of degree $k$ is any complex linear combination of monomials of degree less than or equal to $k$ with at least one non-zero degree $k$ term.

Definition 2.1. For any $k=0,1,2, \ldots$, let $\Lambda_{k}$ be the class of $\lambda \in \Lambda$ such that $\int_{\mathbb{R}^{d}} f d \lambda=$ 0 for polynomials $f$ with degree $\operatorname{deg}(f) \leq k$. Thus, measures in $\Lambda_{k}$ "annihilate" all polynomials of degree up to $k$. We also let $\Lambda_{-1}:=\Lambda$.

Next, we define two operations pertinent to the definition of tangent fields. As usual, for any set $B \subset \mathbb{R}^{d}, c \in \mathbb{R}$ and $s \in \mathbb{R}^{d}$, let $c \cdot B=\{c t: t \in B\}$ and $s+B=B+s=\{s+t$ : $t \in B\}$. Also, define the scaling and translation operations on $\Lambda$ :

$$
r \cdot \lambda:=\lambda\left(r^{-1} \cdot\right), r \neq 0, \quad \text { and } s+\lambda:=\lambda(\cdot-s), \quad s \in \mathbb{R}^{d} .
$$

Clearly, $\Lambda_{k}$ is closed with respect to both of these operations.

Assume that the random elements considered in the paper take values in a complete and separable metric linear space $\left(\mathbb{V}, d_{\mathbb{V}}\right)$ over $\mathbb{C}$. Recall that $\left(\mathbb{V}, d_{\mathbb{V}}\right)$ is said to be a metric linear space (cf. Rolewicz, 1985) if scalar multiplication and addition are continuous with respect to $d_{\mathbb{V}}$. Namely, for all $x_{n}, y_{n}, x, y \in \mathbb{V}$ and $c_{n}, c \in \mathbb{C}$, such that $\left|c_{n}-c\right|+d_{\mathbb{V}}\left(x_{n}, x\right)+d_{\mathbb{V}}\left(y_{n}, y\right) \rightarrow 0$, we have

$$
d_{\mathbb{V}}\left(c_{n} x_{n}, c x\right) \rightarrow 0 \quad \text { and } \quad d_{\mathbb{V}}\left(x_{n}+y_{n}, x+y\right) \rightarrow 0 .
$$

By the Birkhoff-Kakutani Theorem (cf. Theorem 1.1.1 of Rolewicz, 1985), without loss of generality, we can and do assume that the metric $d_{\mathrm{V}}$ is translation invariant, that is,

$$
d_{\mathbb{V}}(x, y)=d_{\mathbb{V}}(x-y, 0) \text { for any } x, y \in \mathbb{V} .
$$

A typical example of $\mathbb{V}$ in our applications is a separable Banach or even Hilbert space. However, we do not restrict to only Banach spaces for now. We also assume throughout that the following continuity condition holds: For any $K>0$,

$$
\lim _{\delta \rightarrow 0} \sup _{|c| \leq K, d_{\mathbb{V}}(x, 0)<\delta} d_{\mathbb{V}}(c x, 0) \rightarrow 0 .
$$

Note that (2.4) readily holds if $\mathbb{V}$ is a normed space and $d_{\mathbb{V}}$ is induced by the norm.

\subsection{The spaces $S\left(\Lambda_{k}, \mathbb{V}\right)$ and $\breve{S}\left(\Lambda_{k}, \mathbb{V}\right)$}

A function $f$ from $\Lambda$ to $\mathbb{V}$ is linear if $f\left(c_{1} \lambda_{1}+c_{2} \lambda_{2}\right)=c_{1} f\left(\lambda_{1}\right)+c_{2} f\left(\lambda_{2}\right), c_{1}, c_{2} \in$ $\mathbb{C}, \lambda_{1}, \lambda_{2} \in \Lambda$. Denote by $S\left(\Lambda_{k}, \mathbb{V}\right)$ the set of all linear functions from $\Lambda_{k}$ to $\mathbb{V}$. In this section, we focus on obtaining a representation of functions in $S\left(\Lambda_{k}, \mathbb{V}\right)$ in terms of functions from $\mathbb{R}^{d}$ to $\mathbb{V}$ as well as a topological structure for a subspace of $S\left(\Lambda_{k}, \mathbb{V}\right)$.

Next we discuss the important notion of representation introduced by Matheron (1973). A function $\breve{f}: \mathbb{R}^{d} \mapsto \mathbb{V}$ is said to be a representation of $f \in S\left(\Lambda_{k}, \mathbb{V}\right)$ if

$$
f(\lambda)=\int \breve{f} d \lambda, \lambda \in \Lambda_{k} .
$$


Consider the following construction of a representation. Denote by $m_{i}, i=1, \ldots, M_{k}:=$ $\left(\begin{array}{c}k+d \\ k\end{array}\right)$ all the monomials of degree less than or equal to $k$ on $\mathbb{R}^{d}$, where the ordering is arbitrary. Define the $\mathbb{R}^{M_{k}}$-valued function

$$
\boldsymbol{b}(t):=\left(m_{1}(t), \ldots, m_{M_{k}}(t)\right)^{\top}, \quad t \in \mathbb{R}^{d} .
$$

Pick the points $t_{i} \in \mathbb{R}^{d}, i=1, \ldots, M_{k}$, such that the $M_{k} \times M_{k}$ matrix $B:=\left(\boldsymbol{b}\left(t_{1}\right), \ldots\right.$, $\left.\boldsymbol{b}\left(t_{M_{k}}\right)\right)$ has full rank. Such points always exist. For instance, if $d=1$ then $B$ has full rank for arbitrary distinct $t_{i}$ 's. However, for $d>1$ some care is needed in selecting the $t_{i}$ to ensure that $B$ has full rank. For $t \in \mathbb{R}^{d}$, define the measure

$$
\lambda_{t}=\delta_{t}-\left(\delta_{t_{1}}, \ldots, \delta_{t_{M_{k}}}\right) B^{-1} \boldsymbol{b}(t),
$$

where, for any $\boldsymbol{c}=\left(c_{1}, \ldots, c_{M_{k}}\right)^{\top} \in \mathbb{C}^{M_{k}},\left(\delta_{t_{1}}, \ldots, \delta_{t_{M_{k}}}\right) c$ denotes the measure $\sum_{j=1}^{M_{k}} c_{j} \delta_{t_{j}}$. Below, for convenience, we adopt such matrix notation when there is no ambiguity. It follows from (2.6) and (2.7) that

$$
\left(\int m_{1} d \lambda_{t}, \ldots, \int m_{M_{k}} d \lambda_{t}\right)^{\top}=\left(I-B B^{-1}\right) \boldsymbol{b}(t)=\mathbf{0},
$$

and so $\lambda_{t} \in \Lambda_{k}$ for all $t$. For any $f \in S\left(\Lambda_{k}, \mathbb{V}\right)$, consider

$$
\breve{f}(t):=f\left(\lambda_{t}\right) .
$$

Note that $\lambda_{t_{i}}=0$, the null measure, for all $i$. Thus,

$$
\left(\breve{f}\left(t_{1}\right), \ldots, \breve{f}\left(t_{M_{k}}\right)\right)=\mathbf{0} .
$$

Moreover, for $\lambda \in \Lambda_{k}$, by linearity,

$$
\int \breve{f} d \lambda=f\left(\lambda-\left(\delta_{t_{1}}, \ldots, \delta_{t_{M_{k}}}\right) B^{-1} \boldsymbol{b}(\lambda)\right)=f(\lambda),
$$

since $\boldsymbol{b}(\lambda)=\mathbf{0}$, and therefore (2.5) holds showing that $\breve{f}$ is a representation of $f$.

Clearly, the function $\breve{f}$ defined by (2.8) is not the only possible representation of $f$. However, any two representations $g_{1}, g_{2}$ of $f$ differ by a polynomial of degree $k$, since, for all $t$,

$$
0=\int\left(g_{1}-g_{2}\right) d \lambda_{t}=g_{1}(t)-g_{2}(t)-\left(g_{1}\left(t_{1}\right)-g_{2}\left(t_{1}\right), \ldots, g_{1}\left(t_{M_{k}}\right)-g_{2}\left(t_{M_{k}}\right)\right) B^{-1} \boldsymbol{b}(t) .
$$

The difference will not affect any of the results in this paper. Thus, from now on, we will adhere to the representation $\breve{f}$ defined by (2.8).

It follows from (2.9) and (2.10) that there is a one-to-one correspondence between $S\left(\Lambda_{k}, \mathbb{V}\right)$ and $\breve{S}\left(\Lambda_{k}, \mathbb{V}\right)$, where $\breve{S}\left(\Lambda_{k}, \mathbb{V}\right)$ denotes the set of functions from $\mathbb{R}^{d}$ to $\mathbb{V}$ that are equal to zero at each $t_{i}, i=1, \ldots, M_{k}$, where the isomorphism is determined by the bijection

$$
\begin{aligned}
(\mathcal{J} f)(t) & =f\left(\lambda_{t}\right), f \in S\left(\Lambda_{k}, \mathbb{V}\right), \\
\left(\mathcal{J}^{-1} \breve{f}\right)(\lambda) & =\int \breve{f} d \lambda, \breve{f} \in \breve{S}\left(\Lambda_{k}, \mathbb{V}\right) .
\end{aligned}
$$

A linear function $f \in S\left(\Lambda_{k}, \mathbb{V}\right)$ is said to be continuous if its representation $\breve{f}$ is a continuous function from $\mathbb{R}^{d}$ to $\mathbb{V}$. By (2.11), this property is "intrinsic" to $f$ and does 
not depend on the representation. Let $S_{c}\left(\Lambda_{k}, \mathbb{V}\right)$ denote the subset of continuous linear functions from $\Lambda_{k}$ to $\mathbb{V}$, and $\breve{S}_{c}\left(\Lambda_{k}, \mathbb{V}\right)$ the corresponding set of representations. The mapping $\mathcal{J}$ in (2.12) continues to be an isomorphism between $S_{c}\left(\Lambda_{k}, \mathbb{V}\right)$ and $\breve{S}_{c}\left(\Lambda_{k}, \mathbb{V}\right)$.

We now proceed to define a topology on $S_{c}\left(\Lambda_{k}, \mathbb{V}\right)$ and $\breve{S}_{c}\left(\Lambda_{k}, \mathbb{V}\right)$. A convenient metric on $\breve{S}_{c}\left(\Lambda_{k}, \mathbb{V}\right)$ is

$$
\rho(\breve{f}, \breve{g})=\sum_{n \geq 1} 2^{-n}\left(1-\exp \left\{-\sup _{\|t\| \leq n} d_{\mathbb{V}}(\breve{f}(t), \breve{g}(t))\right\}\right)
$$

where, as before, $\|\cdot\|$ denotes the Euclidean norm on $\mathbb{R}^{d}$ and $d_{\mathbb{V}}$ denotes the metric on $\mathbb{V}$. Clearly, $\rho$ metrizes the local uniform convergence topology, i.e., uniform convergence on compact sets. We also have the following simple but important fact, proved in Section A.1 in Appendix.

Lemma 2.2. The metric space $\left(\breve{S}_{c}\left(\Lambda_{k}, \mathbb{V}\right), \rho\right)$ is complete and separable.

Using the canonical bijection $\mathcal{J}$, we define the corresponding metric on $S_{c}\left(\Lambda_{k}, \mathbb{V}\right)$ as $\rho(f, g):=\rho(\mathcal{J} f, \mathcal{J} g)$, for $f, g \in S_{c}\left(\Lambda_{k}, \mathbb{V}\right)$, where the same symbol $\rho$ is adopted for convenience. Again, by (2.3), (2.4) and (2.11), the topology so defined does not depend on the particular representation used to define $\left(\breve{S}_{c}\left(\Lambda_{k}, \mathbb{V}\right), \rho\right)$. It follows that $\mathcal{J}$ is an isometry and both $\left(S_{c}\left(\Lambda_{k}, \mathbb{V}\right), \rho\right)$ and $\left(\breve{S}_{c}\left(\Lambda_{k}, \mathbb{V}\right), \rho\right)$ are separable and complete. Thus, weak convergence of probability measures on these spaces can be defined in the usual manner (see, e.g., Billingsley, 1999). Specifically, by Prokhorov's theorem, convergence in distribution in $\left(S_{c}\left(\Lambda_{k}, \mathbb{V}\right), \rho\right)$ or equivalently $\left(\breve{S}_{c}\left(\Lambda_{k}, \mathbb{V}\right), \rho\right)$ is equivalent to the convergence of the finite-dimensional distributions and tightness. The following result provides a general criterion (see also Proposition 2.1 in Falconer, 2002).

Proposition 2.3. Let $X_{n}, X$ be random elements in $\left(S_{c}\left(\Lambda_{k}, \mathbb{V}\right), \rho\right)$ and let $\breve{X}_{n}=\mathcal{J}\left(X_{n}\right)$ and $\breve{X}=\mathcal{J}(X)$. Then $X_{n} \stackrel{d}{\rightarrow} X$ in $\left(S_{c}\left(\Lambda_{k}, \mathbb{V}\right), \rho\right)$, or equivalently $\breve{X}_{n} \stackrel{d}{\rightarrow} \breve{X}$ in $\left(\breve{S}_{c}\left(\Lambda_{k}, \mathbb{V}\right), \rho\right)$, if and only if the following two conditions hold:

(i) For all $m>0$ and $s_{1}, \ldots, s_{m} \in \mathbb{R}^{d}$,

$$
\left(\breve{X}_{n}\left(s_{1}\right), \ldots, \breve{X}_{n}\left(s_{m}\right)\right) \stackrel{d}{\rightarrow}\left(\breve{X}\left(s_{1}\right), \ldots, \breve{X}\left(s_{m}\right)\right) .
$$

(ii) For every compact set $K \subset \mathbb{R}^{d}, \breve{X}_{n}$ is strongly stochastically equicontinuous on $K$, namely, for all $\eta, \epsilon>0$, there exists $\delta>0$ such that

$$
\limsup _{n \rightarrow \infty} \mathbb{P}\left(\sup _{\|s-t\|<\delta, s, t \in K} d_{\mathbb{V}}\left(\breve{X}_{n}(s), \breve{X}_{n}(t)\right)>\eta\right)<\epsilon .
$$

Proof. Since $\left(\breve{S}_{c}\left(\Lambda_{k}, \mathbb{V}\right), \rho\right)$ is separable and complete, the result is a direct consequence of Theorem 14.5 and Proposition 14.6 in Kallenberg (1997).

The convergence of the finite-dimensional distributions (2.14) is often easier to establish, while the challenge is to prove tightness. The following result provides a simple sufficient condition, which also implies the Hölder continuity of the limit. It is a restatement of Corollary 14.9 in Kallenberg (1997).

Proposition 2.4. Suppose that $\breve{X}_{n}, n \in \mathbb{N}$ take values in $\left(\breve{S}_{c}\left(\Lambda_{k}, \mathbb{V}\right), \rho\right)$ and let the sequence of random variables $\left\{\breve{X}_{n}\left(s_{0}\right), n \in \mathbb{N}\right\}$ be tight, for some $s_{0} \in \mathbb{R}^{d}$.

(i) If for some $p>0$ and $\alpha>0$, and all $M>0$, there exist $C_{M}<\infty$, such that

$$
\sup _{n \in \mathbb{N}} \mathbb{E}\left[d_{\mathbb{V}}\left(\breve{X}_{n}(s), \breve{X}_{n}(t)\right)^{p}\right] \leq C_{M}\|s-t\|^{d+\alpha}, \quad \text { for all }\|s\|,\|t\| \leq M, s, t \in \mathbb{R}^{d},
$$

then the laws of the processes $\left\{\breve{X}_{n}, n \in \mathbb{N}\right\}$ are tight in $\left(\breve{S}_{c}\left(\Lambda_{k}, \mathbb{V}\right), \rho\right)$. 
(ii) If, moreover, $\breve{X}_{n} \stackrel{d}{\rightarrow} \breve{X}$, in $\left(\breve{S}_{c}\left(\Lambda_{k}, \mathbb{V}\right), \rho\right)$, then with probability one, $\breve{X}$ has $\gamma$-Hölder continuous paths, for all $\gamma \in(0, \alpha / p)$. That is, there is an event $\Omega^{*}$, with $\mathbb{P}\left(\Omega^{*}\right)=1$, such that for all $M>0$, we have

$$
d_{\mathbb{V}}(\breve{X}(s, \omega), \breve{X}(t, \omega)) \leq C_{M}(\omega)\|s-t\|^{\gamma}, \quad \text { for all }\|s\|,\|t\| \leq M, \omega \in \Omega^{*},
$$

with some $C_{M}(\omega)<\infty$.

Remark 2.5. By taking $\breve{X}_{n} \equiv \breve{X}$ in Proposition 2.4, we recover an extension of the well-known Kolmogorov-Chentsov path-regularity criterion. See also Theorem 2.23 in Kallenberg (1997).

Remark 2.6. If $k \geq 0$, recall that by (2.9), we have $\breve{X}_{n}\left(t_{i}\right)=0, i=1, \cdots, M_{k}$. Thus, in Proposition 2.4, one can trivially take $s_{0}=t_{1}$ and the required tightness of the random variables $\left\{\breve{X}_{n}\left(s_{0}\right), n \in \mathbb{N}\right\}$ is immediate. This condition is non-trivial only when $k=-1$ (the case of stationary processes) where by convention $\mathcal{J}$ is the identity and $\breve{S}_{c}\left(\Lambda_{-1}, \mathbb{V}\right) \equiv S_{c}\left(\Lambda_{-1}, \mathbb{V}\right)$.

The above moment-based criterion is used in Section 5.2 to furnish examples of tangent processes.

\subsection{Scaling actions}

When considering limit theorems for $\mathbb{V}$-valued processes, one may need to rescale the process using an operator different from the usual scalar multiplication. This is particularly relevant for the case where $\mathbb{V}$ is an infinite dimensional space of functions. The next definition introduces the natural conditions that such rescaling operators in a general metric space $\left(\mathbb{X}, d_{\mathbb{X}}\right)$ should possess. It is similar to the one considered in Hult and Lindskog (2006) in their abstract treatment of regular variation. We will later focus on the cases $\mathbb{X}=\mathbb{V}$ and $S_{c}\left(\Lambda_{k}, \mathbb{V}\right)$.

Definition 2.7. Let $\left(\mathbb{X}, d_{\mathbb{X}}\right)$ be a general metric space. A family of (possibly non-linear) operators $T_{a}: \mathbb{X} \rightarrow \mathbb{X}$, indexed by the multiplicative group $\mathbb{R}_{+}:=(0, \infty)$ is said to be a scaling action on $\mathbb{X}$ if the following conditions hold:

(i) for all $a_{1}>0$ and $a_{2}>0$, we have $T_{a_{1}} \circ T_{a_{2}}=T_{a_{1} a_{2}}$,

(ii) $T_{1}$ is the identity, and $T_{a}(0)=0$ for all $a>0$,

(iii) $\left\{T_{a}\right\}$ is continuous, i.e., $d_{\mathbb{X}}\left(T_{a_{n}}\left(x_{n}\right), T_{a}(x)\right) \rightarrow 0$, whenever $a_{n} \rightarrow a>0$ and $d_{\mathbb{X}}\left(x_{n}, x\right) \rightarrow 0$,

(iv) $\left\{T_{a}\right\}$ is radially monotone, i.e., $d_{\mathbb{X}}\left(T_{a_{1}}(x), 0\right)<d_{\mathbb{X}}\left(T_{a_{2}}(x), 0\right)$, for all $0 \neq x \in \mathbb{X}$ and $0<a_{1}<a_{2}$, and

(v) $d_{\mathbb{X}}\left(T_{a}(x), 0\right) \rightarrow 0$ as $a \downarrow 0$, for all $x \in \mathbb{X}$.

The above definition readily implies that $T_{a}, a>0$ are bijections and in particular $T_{a}(x) \neq 0$ for all $x \neq 0$.

Remark 2.8. Property (v) in Definition 2.7 can be replaced by the equivalent condition of

$$
\bigcup_{n=1}^{\infty} T_{n}\left(B_{r}\right)=\mathbb{X} \text { for all } r \in \mathbb{R}_{+},
$$

where $B_{r}:=\left\{x \in \mathbb{X}: d_{\mathbb{X}}(x, 0)<r\right\}$ is the open ball centered at the origin with radius $r$. To see the equivalence, first assume that (2.15) holds and, by (iv), verifying (v) then amounts to showing that $d_{\mathbb{X}}\left(T_{1 / n}(x), 0\right) \rightarrow 0$ for all $x \in \mathbb{X}$. For every $r:=\epsilon>0$, however, (2.15) entails that $x \in T_{n}\left(B_{\epsilon}\right)$ for all sufficiently large $n$. By (i) and (ii), this implies that $T_{1 / n}(x) \in B_{\epsilon}$, or $d_{\mathrm{X}}\left(T_{1 / n}(x), 0\right)<\epsilon$, for all sufficiently large $n$. The converse argument showing (v) implies (2.15) is similar. 
Many common examples of scaling operations readily satisfy the above conditions. For instance, if $\mathbb{X}$ is a linear normed space, a natural scaling action is scalar multiplication itself:

$$
T_{a}(x):=a \cdot x .
$$

More generally, the scalar multiplication is a scaling action if the underlying metric is homogeneous, e.g., $d_{\mathbb{X}}(a \cdot x, a \cdot y)=a^{\gamma} d_{\mathbb{X}}(x, y), \gamma>0$.

Observe that since $T_{1}=\mathrm{I}$ by property (ii), where I stands for the identity operator, we have $T_{a}^{-1}=T_{1 / a}, a>0$ by property (i). Hence the mappings $T_{a}$ are homeomorphisms and map open (closed) sets to open (closed) sets. The radial monotonicity property (iv) implies that $T_{a_{2}}^{-1}\left(B_{r}\right) \subset T_{a_{1}}^{-1}\left(B_{r}\right)$, for all $0<a_{1}<a_{2}$. This, since $T_{a}^{-1}=T_{1 / a}$, entails

$$
T_{a_{1}}\left(B_{r}\right) \subset T_{a_{2}}\left(B_{r}\right), \quad \text { for all } 0<a_{1}<a_{2} .
$$

Note that the metric $d_{\mathrm{X}}$ and the action need not be "compatible", that is, $d_{\mathbb{X}}\left(T_{a}(x), 0\right)$ is in general not equal to $a \cdot d_{\mathbb{X}}(x, 0)$ and therefore, $T_{a}\left(B_{r}\right)$ is in general not $B_{a r}$.

Remark 2.9. In the case where $\mathbb{X}$ is a Hilbert space and $\mathcal{H}: \mathbb{X} \rightarrow \mathbb{X}$ is a fixed bounded linear operator, one can consider the action $T_{c}(x):=c^{\mathcal{H}} x, x \in \mathbb{X}$ for $c>0$, where $c^{\mathcal{H}}:=e^{\log (c) \mathcal{H}}\left(\right.$ see (5.1)). Lemma A.1 shows that $c^{\mathcal{H}}$ is a scaling action in the sense of Definition 2.7, under certain natural conditions on the operator $\mathcal{H}$.

Limit theorems under linear operator scaling on $\mathbb{X}:=\mathbb{R}^{m}$ have been studied extensively in the literature (see e.g. Meerschaert and Scheffler, 2001, and the references therein and thereof). Such actions for a general separable Hilbert space $\mathbb{X}$ will be considered in Section 5.1.

Given a scaling action $\left\{T_{a}, a \in \mathbb{R}_{+}\right\}$on the metric linear space $\left(\mathbb{V}, d_{\mathbb{V}}\right)$, it is natural to consider its coordinate-wise extension on the space of $\mathbb{V}$-valued functions $S\left(\Lambda_{k}, \mathbb{V}\right)$. Namely, the action $\widetilde{T}_{a}: S_{c}\left(\Lambda_{k}, \mathbb{V}\right) \rightarrow S_{c}\left(\Lambda_{k}, \mathbb{V}\right)$ is defined such that for all $f \in S_{c}\left(\Lambda_{k}, \mathbb{V}\right)$, and any $\lambda \in \Lambda_{k}$

$$
\widetilde{T}_{a}(f)(\lambda)=T_{a}(f(\lambda)) .
$$

The following result shows that the coordinate-wise action is in fact a scaling action on $S_{c}\left(\Lambda_{k}, \mathbb{V}\right)$. Its proof is given in Section A.1, below.

Lemma 2.10. For any scaling action $\left\{T_{a}, a \in \mathbb{R}_{+}\right\}$on $\left(\mathbb{V}, d_{\mathbb{V}}\right)$, the coordinate-wise action $\left\{\widetilde{T}_{a}, a \in \mathbb{R}_{+}\right\}$in (2.18) is a scaling action on the linear space $S_{c}\left(\Lambda_{k}, \mathbb{V}\right)$ equipped with the metric $\rho$ in (2.13).

In view of Lemma 2.10, from now on we will use the same notation $\left\{T_{a}\right\}$ for the scaling action on $\mathbb{V}$ and its coordinate-wise extensions on $S_{c}\left(\Lambda_{k}, \mathbb{V}\right)$.

\section{Tangent fields and their properties}

Throughout this section, suppose that $X=\left\{X(\lambda), \lambda \in \Lambda_{k}\right\}$ is a random element in $\left(S_{c}\left(\Lambda_{k}, \mathbb{V}\right), \rho\right)$. That is, for some probability space $(\Omega, \mathcal{F}, \mathbb{P})$, we have that $X: \Omega \rightarrow$ $S_{c}\left(\Lambda_{k}, \mathbb{V}\right)$ is an $\mathcal{F} \mid \mathcal{B}_{\left(S_{c}\left(\Lambda_{k}, \mathrm{~V}\right), \rho\right)}$-measurable map, where $\mathcal{B}_{\left(S_{c}\left(\Lambda_{k}, \mathrm{~V}\right), \rho\right)}$ stands for the Borel $\sigma$-field on $S_{c}\left(\Lambda_{k}\right)$. For $s \in \mathbb{R}^{d}, \lambda \in \Lambda_{k}$ and $r>0$, define

$$
X(s, r \cdot \lambda)=X(s+r \cdot \lambda),
$$

where $s+r \cdot \lambda$ is as defined in (2.2). An example of $X(\lambda)$ is $X(\lambda):=\int_{\mathbb{R}^{d}} X(u) \lambda(d u)$ for some random field $X=\left\{X(s), s \in \mathbb{R}^{d}\right\}$ with continuous sample paths. (Note that in fact every $X$ taking values in $S_{c}\left(\Lambda_{k}, \mathbb{V}\right)$ can be written as $X(\lambda):=\int_{\mathbb{R}^{d}} \breve{X}(u) \lambda(d u)$, where $\breve{X}(u)$ 
is the representation of $X$ as defined in Section 2.1. The integrand is unique up to an additive polynomial of degree no greater than $k$.)

One can interpret $X(s, r \cdot \lambda)$ as a generalized $(1+k)$-th order increment of $X$ at location $s$ and scale $r>0$, relative to $\lambda$. Indeed, consider for example the case $d=1$ and let $\lambda(d u)=\sum_{j=0}^{k+1}\left(\begin{array}{c}k+1 \\ j\end{array}\right)(-1)^{j} \delta_{\{j\}}(d u)$. Then, for $s \in \mathbb{R}$ and $r>0$,

$$
X(s, r \cdot \lambda)=\sum_{j=0}^{k+1}\left(\begin{array}{c}
k+1 \\
j
\end{array}\right)(-1)^{j} X(s+r j)=\Delta_{r}^{k+1} X(s),
$$

where $\Delta_{r} X(s):=X(s+r)-X(s)$ and $\Delta_{r}^{k+1} X(s):=\Delta_{r}\left(\Delta_{r}^{k} X(\cdot)\right)(s), k=0,1, \ldots$ is the usual $(1+k)$-th order regular difference operator.

Thus, considering the process $X$ as a function of $(s+r \cdot \lambda)$, for all (any) signed measures $\lambda \in \Lambda_{k}$ effectively amounts to zooming in on its $(1+k)$ th order increments at location $s$ and scale $r>0$. By letting $r \downarrow 0$, one can examine the local behavior of $X$ and arrive at (generalized) tangent fields as detailed next. This indeed extends the setting of Falconer (2002) who focused on $\mathbb{V}=\mathbb{R}, k=0$, and studied the increment process $X(s+r t)-X(s) \equiv X(s+r \cdot t \lambda), t \in \mathbb{R}$.

One of the goals of the paper is to study the asymptotic behavior of the generalized increments $X(s, r \cdot \lambda)$ as $r \downarrow 0$ for fixed $s \in \mathbb{R}^{d}$, where $r \cdot \lambda$ is as in (2.2). The normalization of the asymptotics will be facilitated by scaling actions $T_{s}=\left\{T_{s, a}, a>0\right\}$ as described by the next definition. In this context, we use that the process $\left\{T_{s, c(s, r)}(X(s, r \cdot \lambda)), \lambda \in \Lambda_{k}\right\}$ is a random element in $S_{c}\left(\Lambda_{k}, \mathbb{V}\right)$ for all $s, r$, which is easy to verify by (3.1) and the continuity of the scaling action. This remark applies to similar situations below and will not be repeated.

Definition 3.1. Let $s \in \mathbb{R}^{d}$. A random process $Y_{s}=\left\{Y_{s}(\lambda), \lambda \in \Lambda_{k}\right\} \in S_{c}\left(\Lambda_{k}, \mathbb{V}\right)$ is said to be a $k$-th order tangent field (or tangent process) to $X$ at $s$ based on the scaling action $T_{s}=\left\{T_{s, a}, a>0\right\}$, if it is non-zero and for some normalizing function $c(s, r)>0$, we have

$$
\left\{T_{s, c(s, r)}(X(s, r \cdot \lambda)), \lambda \in \Lambda_{k}\right\} \stackrel{d}{\longrightarrow}\left\{Y_{s}(\lambda), \lambda \in \Lambda_{k}\right\}, \quad \text { as } r \downarrow 0,
$$

where the convergence in distribution takes place in $\left(S_{c}\left(\Lambda_{k}, \mathbb{V}\right), \rho\right)$.

The role of the function $c(s, r)$ is to provide flexibility in the choice of normalization without having to change the scaling action. For example, in the special setting $\mathbb{V}=$ $\mathbb{R}, k=0$ with the simple scalar scaling action $T_{s, a}(x) \equiv T_{a}(x)=a \cdot x$, and $\lambda$ replaced by $\lambda_{t}(d x):=\delta_{\{t\}}(d x)-\delta_{\{0\}}(d x)$, Relation (3.2) implies

$$
\{c(s, r)(X(s+r t)-X(s)), t \in \mathbb{R}\} \stackrel{d}{\longrightarrow}\left\{Y_{s}\left(\lambda_{t}\right), t \in \mathbb{R}\right\}, \quad \text { as } r \downarrow 0 .
$$

This recovers the classic setting, of tangent processes, where $c(s, r)$ plays the role of a normalizing constant. In this case, Falconer (2002) showed that tangent fields must be self-similar and have stationary increments. In the following two subsections, we extend Falconer's results to the general setting of this paper.

\subsection{Tangent fields are self-similar}

Self-similarity is a distributional invariance phenomenon, which is ubiquitous in the study of stochastic process limit theory. Recall that a real-valued stochastic process $\xi=\left\{\xi(t), t \in \mathbb{R}^{d}\right\}$ is said to be self-similar with self-similarity exponent $H>0$, if for all $r>0$, we have $\left\{\xi(r t), t \in \mathbb{R}^{d}\right\} \stackrel{f d d}{=}\left\{r^{H} \xi(t), t \in \mathbb{R}^{d}\right\}$, where $\stackrel{f d d}{=}$ means equality of all finite-dimensional distributions. For $\mathbb{V}$-valued processes, we have the following natural extension of the notion of self-similarity. 
Tangent fields, intrinsic stationarity, and self similarity

Definition 3.2. An $\mathbb{V}$-valued stochastic process $\xi=\left\{\xi(\lambda), \lambda \in \Lambda_{k}\right\}$ is said to be selfsimilar relative to the scaling action $\left\{T_{a}\right\}$ if for some $\alpha \in \mathbb{R}$, we have

$$
\left\{\xi(r \cdot \lambda), \lambda \in \Lambda_{k}\right\} \stackrel{f d d}{=}\left\{T_{r^{\alpha}}(\xi(\lambda)), \lambda \in \Lambda_{k}\right\}, \quad \text { for all } r>0 .
$$

Remark 3.3. The above definition views $\xi$ in the wide sense as a measurable map $\xi: \Omega \rightarrow \mathbb{V}^{\Lambda_{k}}$, where $\mathbb{V}^{\Lambda_{k}}$ is equipped with the product $\sigma$-field $\mathcal{B}_{\mathbb{V}^{\Lambda_{k}}}$ generated by the class $\mathcal{C}$ all finite-dimensional cylinder sets $C:=\left\{x \in \mathbb{V}^{\Lambda_{k}}: x\left(\lambda_{i}\right) \in B_{i}, i=\right.$ $1, \cdots, m\}, B_{i} \in \mathcal{B}_{\left(\mathbb{V}, d_{\mathbb{V}}\right)}, \lambda_{i} \in \Lambda_{k}, i=1, \cdots, m, m \in \mathbb{N}$. When the paths of $\xi$ are continuous, i.e., $\xi$ is a random element in $\left(S_{c}\left(\Lambda_{k}, \mathbb{V}\right), \rho\right)$, it can be shown that the equality of the finite-dimensional distributions " $\stackrel{f d d}{=}$ " in (3.3) is equivalent to equality in distribution “䇂” between $S_{c}\left(\Lambda_{k}, \mathbb{V}\right)$-valued random elements. Indeed, firstly, all finite-dimensional projections $\pi_{\lambda_{1}, \cdots, \lambda_{m}}: S_{c}\left(\Lambda_{k}, \mathbb{V}\right) \rightarrow \mathbb{V}^{m}, m \in \mathbb{N}$ for $\lambda_{i} \in \Lambda_{k}, i=1, \cdots, m$ are continuous and hence $\mathcal{B}_{\left(S_{c}\left(\Lambda_{k}, \mathbb{V}\right), \rho\right)}$-measurable. Since $\mathcal{B}_{\mathbb{V}^{\Lambda_{k}}}$ is the minimal $\sigma$-filed making all such projections measurable, we obtain that $S_{c}\left(\Lambda_{k}, \mathbb{V}\right) \cap \mathcal{B}_{\mathbb{V}^{\Lambda_{k}}} \subset \mathcal{B}_{\left(S_{c}\left(\Lambda_{k}, \mathbb{V}\right), \rho\right) \text {, where }}$

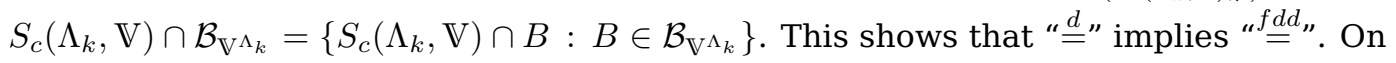
the other hand, the fact that $\left(S_{c}\left(\Lambda_{k}, \mathbb{V}\right), \rho\right)$ is second-countable, entails that $\mathcal{B}_{\left(S_{c}\left(\Lambda_{k}, \mathbb{V}\right), \rho\right)}$ is generated by the class of all closed balls, for example. Since each such ball is a countable intersection of cylinder sets (e.g., as in the proof of Proposition 12.2.2 in Dudley, 2002) it follows that the Borel $\sigma$-field $\mathcal{B}_{\left(S_{c}\left(\Lambda_{k}, \mathrm{~V}\right), \rho\right)}$ is determined by the $\pi$-system $\mathcal{C}$ of all finite-dimensional cylinder sets (restricted to $S_{c}\left(\Lambda_{k}, \mathbb{V}\right)$ ). Thus, appealing to the $\pi-\lambda$ Theorem, we see that " $\stackrel{f d d}{=}$ " implies also " $=$ ".

The seminal work of Lamperti (1962) shows that all non-trivial large-scale limits of stochastically continuous processes are self-similar. From this perspective, it is expected that tangent fields (as small-scale limits) be self-similar. Falconer $(2002,2003)$ has shown that this is indeed the case for $k=0$. The next result addresses the general case of $k$-th order tangent fields of $\mathbb{V}$-valued processes.

Theorem 3.4. Assume that, for some location $s \in \mathbb{R}^{d},\left\{Y_{s}(\lambda), \lambda \in \Lambda_{k}\right\}$ is a $k$-th order tangent field to $X$ at $s$ with respect to the scaling action $T_{s}$. That is, Relation (3.2) holds for some $c(s, r)$.

(i) Then, for all $r>0$, we have

$$
\left\{Y_{s}(r \cdot \lambda), \lambda \in \Lambda_{k}\right\} \stackrel{d}{=}\left\{T_{r^{\alpha(s)}} Y_{s}(\lambda), \lambda \in \Lambda_{k}\right\},
$$

where $\alpha(s)>0$ is some positive constant. We have, moreover, that

$$
c(s, r)=r^{-\alpha(s)} \ell_{s}(r),
$$

where $\ell_{s}(r)$ is a slowly varying function at 0 , i.e., for every fixed $h>0, \ell_{s}(h r) / \ell_{s}(r) \rightarrow$ $1, r \downarrow 0$.

(ii) The tangent process is unique up to rescaling. That is, if (3.2) also holds with $c(s, r)$ and $Y_{s}=\left\{Y_{s}(\lambda)\right\}$ replaced by $\widetilde{c}(s, r)$ and $\widetilde{Y}_{s}=\left\{\widetilde{Y}_{s}(\lambda)\right\}$, respectively, then we have

$$
\lim _{r \downarrow 0} \frac{\widetilde{c}(s, r)}{c(s, r)}=a \in(0, \infty) \quad \text { and } \quad \widetilde{Y}_{s} \stackrel{d}{=} T_{s, a}\left(Y_{s}\right) .
$$

Remark 3.5. Relations (3.4) and (3.5) show that the normalization used to define a tangent field may differ from the scaling action that characterizes the self-similarity of the tangent field by a slowly varying factor, which cannot be dropped in general. This is akin to the fundamental role of slowly varying functions in the normalization of the partial sums in the non-Gaussian Central Limit Theorem. 
Proof of Theorem 3.4: Proof of part (i). For all fixed $h>0$, by (3.2), as $r \downarrow 0$, we have

$$
\widetilde{\xi}_{r}:=\left\{T_{s, c(s, r)}(X(s,(h r) \cdot \lambda)), \lambda \in \Lambda_{k}\right\} \stackrel{d}{\longrightarrow} \widetilde{\xi}:=\left\{Y_{s}(h \cdot \lambda), \lambda \in \Lambda_{k}\right\} .
$$

On the other hand, as $r \downarrow 0$,

$$
\xi_{r}:=\left\{T_{s, c(s, h r)}(X(s,(h r) \cdot \lambda)), \lambda \in \Lambda_{k}\right\} \stackrel{d}{\longrightarrow} \xi:=\left\{Y_{s}(\lambda), \lambda \in \Lambda_{k}\right\} .
$$

By assumption both $\xi$ and $\widetilde{\xi}$ are non-zero. Observe that

$$
T_{s, c(s, r)}(X(s,(h r) \cdot \lambda))=T_{s, \frac{c(s, r)}{c(s, h r)}} \circ T_{s, c(s, h r)}(X(s,(h r) \cdot \lambda))
$$

and hence

$$
T_{s, \frac{c(s, r)}{c(s, h r)}}\left(\xi_{r}\right)=\widetilde{\xi}_{r} \stackrel{d}{\rightarrow} \widetilde{\xi}
$$

Applying Lemma A.6 (with $\mathbb{X}:=S_{c}\left(\Lambda_{k}, \mathbb{V}\right)$ - recall Lemma 2.10) gives

$$
\frac{c(s, r)}{c(s, h r)} \rightarrow a(s, h), \quad \text { as } r \downarrow 0,
$$

for some positive $a(s, h)>0$. We have, moreover, $\widetilde{\xi} \stackrel{d}{=} T_{s, a(s, h)}(\xi)$, which reads

$$
\left\{Y_{s}(h \cdot \lambda), \lambda \in \Lambda_{k}\right\} \stackrel{d}{=}\left\{T_{s, a(s, h)}\left(Y_{s}(\lambda)\right), \lambda \in \Lambda_{k}\right\} .
$$

We will next show that $a(s, h)=h^{\alpha(s)}$, for some $\alpha(s)>0$. First, Relation (3.8) readily implies that for all $h_{1}>0$ and $h_{2}>0$

$$
a\left(s, h_{1} h_{2}\right)=a\left(s, h_{1}\right) a\left(s, h_{2}\right) .
$$

Indeed, by (3.8),

$$
\begin{aligned}
\left\{T_{s, a\left(s, h_{1} h_{2}\right)}\left(Y_{s}(\lambda)\right)\right\} & \stackrel{d}{=}\left\{Y_{s}\left(\left(h_{1} h_{2}\right) \cdot \lambda\right)\right\} \stackrel{d}{=}\left\{T_{s, a\left(s, h_{1}\right)}\left(Y_{s}\left(h_{2} \cdot \lambda\right)\right)\right\} \\
& \stackrel{d}{=}\left\{T_{s, a\left(s, h_{1}\right)} \circ T_{s, a\left(s, h_{2}\right)}\left(Y_{s}(\lambda)\right)\right\}=\left\{T_{s, a\left(s, h_{1}\right) a\left(s, h_{2}\right)}\left(Y_{s}(\lambda)\right)\right\} .
\end{aligned}
$$

Since $Y_{s}$ is nonzero, the last relation implies (3.9) by (i) of Lemma A.6.

The function $a(s, h)$ is also continuous in $h \in(0, \infty)$. Indeed, for any sequence $h_{n} \rightarrow h$, $h_{n}, h \in(0, \infty)$, by Lemma A.7 (applied with $X_{n}:=Y_{s}, v_{n}:=0$, and $r_{n}:=h_{n}$ ), we have $\left\{Y_{s}\left(h_{n} \cdot \lambda\right)\right\} \stackrel{d}{\rightarrow}\left\{Y_{s}(h \cdot \lambda)\right\}$. Therefore, by (3.8),

$$
\left\{T_{s, a\left(s, h_{n}\right)}\left(Y_{s}(\lambda)\right)\right\} \stackrel{d}{=}\left\{Y_{s}\left(h_{n} \cdot \lambda\right)\right\} \stackrel{d}{\longrightarrow}\left\{Y_{s}(h \cdot \lambda)\right\} \stackrel{d}{=}\left\{T_{s, a(s, h)}\left(Y_{s}(\lambda)\right)\right\} .
$$

Since $Y_{s}$ is nonzero, applying (ii) of Lemma A.6, we obtain

$$
a\left(s, h_{n}\right) \rightarrow a(s, h),
$$

which shows the desired continuity.

Combining (3.9), (3.11), the continuity of $a(s, \cdot)$ and the fact that, trivially, $a(s, 1)=1$, it is straightforward to conclude that $a(s, h)=h^{\alpha(s)}, h>0$, for some $\alpha(s) \in(-\infty, \infty)$, which is a special example of Cauchy's functional equation (cf. Theorem 5.2.1 of Kuczma, 2009).

We will show next that $a\left(s, h_{n}\right) \rightarrow 0$ as $h_{n} \downarrow 0$, which necessarily implies $\alpha(s)>0$. Indeed, with $h=0$, (3.10) implies that $\left\{T_{s, a\left(s, h_{n}\right)}\left(Y_{s}(\lambda)\right)\right\} \stackrel{d}{\rightarrow} 0=\left\{Y_{s}(0 \cdot \lambda)\right\}$, as $n \rightarrow \infty$. This, by (iii) of Lemma A.6, yields $a\left(s, h_{n}\right) \rightarrow 0$. 
To conclude the proof of part (i), letting $c(s, r)=: r^{-\alpha(s)} \ell_{s}(r)$, we see from Equation (3.7) that, for all $h>0, \ell_{s}(r) / \ell_{s}(h r) \rightarrow 1$, as $r \downarrow 0$, which shows $\ell_{s}$ is a slowly varying function at 0 .

Proof of part (ii). Assume now that in addition to (3.2), we have

$$
\widetilde{\eta}_{r}:=\left\{T_{s, \widetilde{c}(s, r)}(X(s, r \cdot \lambda)), \lambda \in \Lambda_{k}\right\} \stackrel{d}{\longrightarrow} \widetilde{Y}_{s}=\left\{\widetilde{Y}_{s}(\lambda), \lambda \in \Lambda_{k}\right\},
$$

as $r \downarrow 0$. By the properties of the scaling action, with $\eta_{r}:=\left\{T_{s, c(s, r)}(X(s, r \cdot \lambda)), \lambda \in \Lambda_{k}\right\}$, we have

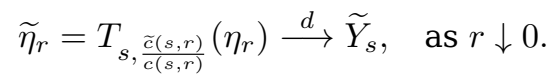

On the other hand, Relation (3.2) reads $\eta_{r} \stackrel{d}{\rightarrow} Y_{s}$, as $r \downarrow 0$. Since both limits $\widetilde{Y}_{s}$ and $Y_{s}$ are non-zero, Lemma A.6 entails $\widetilde{c}(s, r) / c(s, r) \rightarrow a>0$ and $T_{s, a}\left(Y_{s}\right) \stackrel{d}{=} \widetilde{Y}_{s}$, which proves (3.6), i.e., the essential uniqueness of the tangent process.

\subsection{Tangent fields are intrinsically stationary}

One of the key results in Falconer (2002) is that (almost all) tangent fields have stationary increments (cf. Theorem 3.6 therein). The proof of that is based on a delicate measure-theoretic argument. Below, we show that this phenomenon extends to higher order tangent fields to processes taking values in a linear separable metric space $\mathbb{V}$.

Let (3.2) hold and let

$$
F_{n}(s):=\text { Law of }\left\{T_{s, c(s, 1 / n)} X(s,(1 / n) \cdot \lambda), \lambda \in \Lambda_{k}\right\}
$$

be the probability distribution of the rescaled version of $\left\{X(s, \lambda), \lambda \in \Lambda_{k}\right\}$ in $\left(S_{c}\left(\Lambda_{k}, \mathbb{V}\right), \rho\right)$. Similarly, let

$$
G(s):=\operatorname{Law} \text { of }\left\{Y_{s}(\lambda), \lambda \in \Lambda_{k}\right\} .
$$

In this notation, the convergence in (3.2) (with $r:=1 / n$ ) is simply

$$
F_{n}(s) \stackrel{w}{\longrightarrow} G(s), \quad n \rightarrow \infty,
$$

where ' $\stackrel{w}{\rightarrow}$ ' denotes the weak convergence of probability measures. An important result that will be utilized below is Proposition 3.9 in the Appendix. In that regard, we first equip the space $\mathcal{P}\left(S_{c}\left(\Lambda_{k}, \mathbb{V}\right), \rho\right)$ of probability measures on $\left(S_{c}\left(\Lambda_{k}, \mathbb{V}\right), \rho\right)$ with a separable metric that metrizes the weak convergence (3.14). Since $\left(S_{c}\left(\Lambda_{k}, \mathbb{V}\right), \rho\right)$ is complete and separable, a suitable metric is $d_{\mathrm{LP}}$, the Lévy-Prokhorov distance (cf. Theorem 6.8 of Billingsley, 1999). Thus, (3.14) can be re-expressed as

$$
F_{n}(s) \rightarrow G(s) \text { in }\left(\mathcal{P}\left(S_{c}\left(\Lambda_{k}, \mathbb{V}\right), \rho\right), d_{\mathrm{LP}}\right),
$$

namely, $d_{\mathrm{LP}}\left(F_{n}(s), G(s)\right) \rightarrow 0$.

Definition 3.6. A process $Y=\left\{Y(\lambda), \lambda \in \Lambda_{k}\right\}$ is said to be strictly intrinsically stationary if

$$
\left\{Y(w+\lambda), \lambda \in \Lambda_{k}\right\} \stackrel{f d d}{=}\left\{Y(\lambda), \lambda \in \Lambda_{k}\right\}, \quad \text { for all } w \in \mathbb{R}^{d} .
$$

Note that this is different from the usual notion of weak or second-order intrinsic stationarity in the literature (cf. Sasvári, 2009). The latter is the topic of Section 4. 
Remark 3.7. Observe that the notion of strict intrinsic stationarity like that of selfsimilarity in (3.3) is stated in greater generality using equality in the sense of finitedimensional distributions. As discussed in Remark 3.3, when the processes therein take values in the path space $S_{c}\left(\mathbb{R}^{d}, \mathbb{V}\right)$, the equality of the finite-dimensional distributions is equivalent to that of the probability distributions of the processes. In the next result, the processes are understood as random elements in $S_{c}\left(\mathbb{R}^{d}, \mathbb{V}\right)$.

Theorem 3.8. Let $B$ be a Borel set of $\mathbb{R}^{d}$. Assume that $X=\left\{X(\lambda), \lambda \in \Lambda_{k}\right\}$ is a random element in $S_{c}\left(\mathbb{R}^{d}, \mathbb{V}\right)$ and it has a $k$-th order tangent field $Y_{s}=\left\{Y_{s}(\lambda), \lambda \in \Lambda_{k}\right\}$ at every $s \in B$ in the sense of Definition 3.1. Also assume that for any $r$, the normalization $c(s, r)$ is Borel measurable in $s$, and for any $s$ and any sequence $w_{n} \rightarrow w \in \mathbb{R}^{d}$,

$$
T_{s_{n}, c\left(s_{n}, 1 / n\right)} \circ T_{s, c(s, 1 / n)}^{-1} \rightarrow \mathrm{I},
$$

where I is the identity operator and $s_{n}:=s+n^{-1} w_{n}$. Then, there exists a set $U$ with zero Lebesgue measure such that for all $s \in B \backslash U$, the tangent field $Y_{s}$ is strictly intrinsically stationary. That is, at almost all locations $s$, tangent fields are strictly intrinsically stationary.

The proof of this result uses the following proposition established in Section A.2 below.

Proposition 3.9. Let $B \subset \mathbb{R}^{d}$ be a Borel set with finite Lebesgue measure $\operatorname{Leb}(B)<\infty$. Suppose that $F_{n}: B \rightarrow E$ is a sequence of Borel measurable functions into the separable metric space $\left(E, \rho_{E}\right)$ such that

$$
F_{n}(s) \underset{n \rightarrow \infty}{\longrightarrow} G(s), \text { for almost all } s \in B .
$$

Then, for every $\epsilon>0$, there exists a compact set $K_{\epsilon} \subset B$, such that $\operatorname{Leb}\left(B \backslash K_{\epsilon}\right)<\epsilon$, the function $G$ being continuous on $K_{\epsilon}$, and

$$
F_{n}\left(s_{n}\right) \rightarrow G(s), \text { whenever } s_{n} \rightarrow s, \text { for } s_{n}, s \in K_{\epsilon} .
$$

Proof of Theorem 3.8: By the $\sigma$-additivity of the Lebesgue measure on $\mathbb{R}^{d}$, it suffices to establish the result for the case $\operatorname{Leb}(B)<\infty$.

The assumption implies that (3.15) holds for all $s \in B$. The continuity of $X$ and the Borel-measurability of $s \mapsto c(s, 1 / n)$ entail that $s \mapsto F_{n}(s)$ is a sequence of Borel measurable functions in $\left(E, \rho_{E}\right):=\left(\mathcal{P}\left(S_{c}\left(\Lambda_{k}, \mathbb{V}\right), \rho\right), d_{\mathrm{LP}}\right)$. Therefore, the assumptions of Proposition 3.9 are fulfilled and for any $\epsilon>0$, there is a compact set $K_{\epsilon} \subset B$ with $\operatorname{Leb}\left(B \backslash K_{\epsilon}\right)<\epsilon$ such that $F_{n}\left(s_{n}\right) \rightarrow G(s)$ so long as $s_{n}, s \in K_{\epsilon}$ and $s_{n} \rightarrow s$.

It follows from Lebesgue's density theorem that there is a subset $K_{\epsilon}^{\prime}$ of $K_{\epsilon}$ on which the Lebesgue density is equal to 1 and $\operatorname{Leb}\left(K_{\epsilon} \backslash K_{\epsilon}^{\prime}\right)=0$. By Lemma 3.5 of Falconer (2002), for any $s \in K_{\epsilon}^{\prime}$ and $w \in \mathbb{R}^{d}$, there exists a sequence $w_{n}$ such that $w_{n} \rightarrow w$ and $s_{n}:=s+w_{n} / n \in K_{\epsilon}$. Thus, for any $s \in K_{\epsilon}^{\prime}, F_{n}\left(s_{n}\right) \rightarrow G(s)$ as $n \rightarrow \infty$ or, equivalently,

$$
\xi_{n}:=\left\{T_{s_{n}, c\left(s_{n}, 1 / n\right)}\left(X\left(s_{n},(1 / n) \cdot \lambda\right)\right), \lambda \in \Lambda_{k}\right\} \stackrel{d}{\longrightarrow} \xi:=\left\{Y_{s}(\lambda), \lambda \in \Lambda_{k}\right\},
$$

On the other hand, we have

$$
\begin{aligned}
\xi_{n} & =\left\{T_{s_{n}, c\left(s_{n}, 1 / n\right)}\left(X\left(s_{n},(1 / n) \cdot \lambda\right), \lambda \in \Lambda_{k}\right\}\right. \\
& =\left\{T_{s_{n}, c\left(s_{n}, 1 / n\right)} \circ T_{s, c(s, 1 / n)}^{-1} \circ T_{s, c(s, 1 / n)}\left(X\left(s_{n},(1 / n) \cdot \lambda\right)\right), \lambda \in \Lambda_{k}\right\} \\
& =\left\{T_{s_{n}, c\left(s_{n}, 1 / n\right)} \circ T_{s, c(s, 1 / n)}^{-1} \circ T_{s, c(s, 1 / n)}\left(X\left(s,(1 / n) \cdot\left(w_{n}+\lambda\right)\right)\right), \lambda \in \Lambda_{k}\right\} .
\end{aligned}
$$


Since by (3.16), we have $T_{s_{n}, c\left(s_{n}, 1 / n\right)} \circ T_{s, c(s, 1 / n)}^{-1} \rightarrow \mathrm{I}$, Lemma A.7 implies

$$
\xi_{n} \stackrel{d}{\longrightarrow} \widetilde{\xi}:=\left\{Y_{s}(w+\lambda), \lambda \in \Lambda_{k}\right\},
$$

which implies that $\xi \stackrel{d}{=} \widetilde{\xi}$.

Finally, we take $U:=\cap_{k=1}^{\infty}\left(B \backslash K_{1 / n}^{\prime}\right)$, which is a set with measure 0 . This concludes the proof.

We make next an important observation that Condition (3.16) holds automatically in the case when the scaling actions $T_{s, c}$ can be expressed through a single scaling action independent of the location $s$. This is the case in particular for the usual scalar multiplication actions (2.16) considered for example in (cf. Falconer, 2002).

Corollary 3.10. Assume the conditions of Theorem 3.8. If the scaling action does not depend on location, i.e., $T_{s, c(s, r)}=T_{c(s, r)}, c>0$, then Condition (3.16) always holds.

The proof is given in Section A.3. We conclude this section with several remarks.

Remark 3.11. The null set $U$ in Theorem 3.8 cannot be dropped in general. While all tangent fields are self-similar, not all of them are intrinsically stationary. Indeed, one can consider the simple example $X(t)=\|t\|^{H} Z, t \in \mathbb{R}^{d}$, where $Z$ is a fixed random variable and $H>0$. Consider the usual scalar multiplication action and observe that with $s:=0$, and $\lambda=\sum_{i} c_{i} \delta_{t_{i}} \in \Lambda_{k}$, for all $r>0$, we have

$$
X(s+r \cdot \lambda)=\sum_{i} c_{i} X\left(r t_{i}\right)=r^{H} \sum_{i} c_{i} X\left(t_{i}\right)=r^{H} X(s+\lambda) .
$$

That is, $X$ is its own tangent field at $s=0$ for all $k \geq 0$. Note that $X$ is not intrinsically stationary if $H \notin \mathbb{N}$.

Remark 3.12. Notice that $\Lambda_{k_{2}} \subset \Lambda_{k_{1}}$ for all $0 \leq k_{1}<k_{2}$. Therefore, all $k_{1}$-order tangent fields are also $k_{2}$-order tangent fields. Specifically, if (3.2) holds with $k=k_{1}$, then it also holds with $k_{2}$.

Remark 3.13. As in Falconer (2002), we focus here on random fields with continuous paths. One can study the structure of generalized tangent fields for processes with discontinuous paths and potentially extend the results in Falconer (2003) which focus on the space of càdlág functions equipped with the Skorokhod $J_{1}$-topology. The key challenge is coming up with a suitable topology on the path-space in question which is separable and complete. Provided that this is the case, we believe that versions of Theorems 3.4 and 3.8 will continue to hold.

Remark 3.14. In principle, in the definition of the tangent field (3.2) one could apply a general scaling action on both the domain $\mathbb{R}^{d}$ of the stochastic process as well as on its range $\mathbb{V}$. In this case, we anticipate that an analog of Theorem 3.4 will hold, where the limits are scale-invariant processes similar to the ones studied in Biermé et al. (2007); Didier et al. (2017). Here, for simplicity, we chose to apply a general scaling action only on the range of the process and retain the usual rescaling by scalars in the domain $\mathbb{R}^{d}$.

\section{Spectral theory for Hilbert space valued IRF $_{k}$ 's}

In this section, we develop the general correlation theory for stationary and intrinsically stationary processes taking values in a separable Hilbert space $\mathbb{V}$ over $\mathbb{C}$ equipped with the inner product $\langle\cdot, \cdot\rangle$. In the following section, we present a generalization of the celebrated Bochner Theorem and then in Section 4.2, we extend the Matheron spectral characterization to the class of $\mathbb{V}$-valued intrinsic random functions. The applications of these results to the characterization of Gaussian $\mathbb{V}$-valued stationary and intrinsically 
stationary processes requires us to carefully consider both real and complex Hilbert spaces (discussed in Section 4.3).

Throughout this paper, a random element $X$ in $\mathbb{V}$ is said to have mean zero and finite variance, together referred to as second order, if $\mathbb{E}[X]=0$ and $\mathbb{E}\left[\|X\|^{2}\right]<\infty$, where, for definiteness, all expectations here are defined in the Bochner sense (see Section S.2.1 in Shen et al., 2020). A process is said to be second order if each element is second order.

Denote by $\mathbb{T}$ the collection of trace-class operators on $\mathbb{V}$. That is, linear operators $\mathcal{T}: \mathbb{V} \rightarrow \mathbb{V}$, with finite trace norm:

$$
\|\mathcal{T}\|_{\text {tr }}=\sum_{j=1}^{\infty}\left\langle\left(\mathcal{T}^{*} \mathcal{T}\right)^{1 / 2} e_{j}, e_{j}\right\rangle
$$

where $\left\{e_{j}\right\}$ is an arbitrary complete orthonormal system (CONS) on $\mathbb{V}$, and where $\mathcal{T}^{*}$ denotes the adjoint operator of $\mathcal{T}$. One can show that the trace norm does not depend on the choice of the CONS and the space $\mathbb{T}$ equipped with the trace norm is a Banach space (cf. Simon, 2015).

Recall that $\mathcal{T}$ is self-adjoint if $\mathcal{T}=\mathcal{T}^{*}$. Also $\mathcal{T}$ is positive definite (or just positive), denoted $\mathcal{T} \geq 0$, if $\mathcal{T}$ is self-adjoint and $\langle f, \mathcal{T} f\rangle \geq 0$, for all $f \in \mathbb{V}$. The class of positive and trace-class operators will be denoted by $\mathbb{T}_{+}$.

\subsection{The Bochner Theorem}

The aim of this subsection is to review the basic properties of second order covariancestationary processes on $\mathbb{R}^{d}$ taking values in the separable Hilbert space $\mathbb{V}$ over $\mathbb{C}$. We start with the important notion of positive definiteness.

Definition 4.1. A collection of operators $\left\{\mathcal{K}(t), t \in \mathbb{R}^{d}\right\}$ on the complex Hilbert space $\mathbb{V}$ is said to be positive definite in the weak sense if for all $c_{j} \in \mathbb{C}, t_{j} \in \mathbb{R}^{d}, j=1, \cdots, n$, we have

$$
\sum_{j=1}^{n} \sum_{j^{\prime}=1}^{n} c_{j} \bar{c}_{j^{\prime}} \mathcal{K}\left(t_{j}-t_{j^{\prime}}\right) \geq 0 \text { (operator positivity). }
$$

The classical Bochner's Theorem (cf. Bochner, 1948; Khintchine, 1934) which connects the space of positive-definite functions with range in $\mathbb{C}$ and finite positive measures has provided a fundamental tool for constructing useful models for stationary random fields. Below we state an extension of that for the infinite-dimensional setting. To do so we need the notion of integration with respect to a $\mathbb{T}_{+}$-valued measure.

We say that $\mu: \mathcal{B}\left(\mathbb{R}^{d}\right) \mapsto \mathbb{T}_{+}$is a $\mathbb{T}_{+}$-valued measure if $\mu$ is $\sigma$-additive, where $\mathcal{B}\left(\mathbb{R}^{d}\right)$ denotes the $\sigma$-field of Borel sets in $\mathbb{R}^{d}$. Note that a fortiori $\mu(\emptyset)=0$ and $\mu$ is finite in the sense that $0 \leq \mu(B) \leq \mu\left(\mathbb{R}^{d}\right) \in \mathbb{T}_{+}, B \in \mathcal{B}\left(\mathbb{R}^{d}\right)$ as positive operators. Integration of a $\mathbb{C}$-valued measurable function with respect to such $\mu$ can be defined along the line of Lebesgue integration; see Section A.4.

Theorem 4.2. Let $\left\{\mathcal{K}(t), t \in \mathbb{R}^{d}\right\} \subset \mathbb{T}$ be a positive-definite set of trace-class operators in the sense of Definition 4.1. If $\mathcal{K}$ is continuous at 0 in the trace norm, i.e., $\|\mathcal{K}(t)-\mathcal{K}(0)\|_{\operatorname{tr}} \rightarrow$ 0 , as $t \rightarrow 0$, then there exists a unique finite $\mathbb{T}_{+}$-valued measure $\mu$ such that

$$
\mathcal{K}(t)=\int_{\mathbb{R}^{d}} e^{i t^{\top} x} \mu(d x), \quad \text { for all } t \in \mathbb{R}^{d} .
$$

Conversely, for every finite $\mathbb{T}_{+}$-valued measure $\mu$, Relation (4.2) yields a positive-definite set of trace class operators.

We note that Theorem 4.2 or variations of it have been mentioned in the literature. See, for instance, Kallianpur and Mandrekar (1971), Holmes (1979), Neeb (1998), 
Durand and Roueff (2020) and van Delft and Eichler (2020). In Section S.4.1 of Shen et al. (2020), we provide a detailed proof that uses standard arguments familiar to the readers in the statistics and probability community.

Both Neeb (1998) and van Delft and Eichler (2020) present Bochner's Theorem in terms of the following natural but stronger version of positivity.

Definition 4.3. A collection of operators $\left\{\mathcal{K}(t), t \in \mathbb{R}^{d}\right\}$ on $\mathbb{V}$ is said to be completely positive definite, or just positive definite, if

$$
\sum_{j=1}^{n} \sum_{j^{\prime}=1}^{n}\left\langle f_{j}, \mathcal{K}\left(t_{j}-t_{j^{\prime}}\right) f_{j^{\prime}}\right\rangle \geq 0,
$$

for all $f_{j} \in \mathbb{V}, t_{j} \in \mathbb{R}^{d}, j=1, \cdots, n$ and $n \in \mathbb{N}$.

Definition 4.3 simply means that the matrices $\left(\mathcal{K}\left(t_{j}-t_{j^{\prime}}\right)\right)_{n \times n}$ with operator $\mathbb{T}$-valued entries are self-adjoint positive definite operators on the product Hilbert space $\mathbb{V}^{n}$. For more mathematical insight into this condition, see abstract literature on Hilbert $C^{*}$-modules, e.g., Murphy (1997) and Pellonpää and Ylinen (2011). Clearly (4.3) implies (4.1). However, observe that for every finite $\mathbb{T}_{+}$-valued measure $\mu$, Relation (4.2) defines a completely positive definite kernel $\mathcal{K}(\cdot)$. This entails the following curious result, already noted in Durand and Roueff (2020).

Corollary 4.4. Let $\mathcal{K}: \mathbb{R}^{d} \rightarrow \mathbb{T}$ be continuous at 0 in $\|\cdot\|_{\mathrm{tr}}$. The collection of operators $\left\{\mathcal{K}(t), t \in \mathbb{R}^{d}\right\}$ is positive definite in the sense of Definition 4.1 if and only if it is completely positive definite in the sense of Definition 4.3.

Let now $\left\{X(t), t \in \mathbb{R}^{d}\right\}$ be a $\mathbb{V}$-valued, second order random field. The cross covariance operator for $X$ is then well-defined as

$$
\mathcal{C}_{X}(s, t):=\mathbb{E}[X(s) \otimes X(t)], \quad s, t \in \mathbb{R}^{d},
$$

and takes values in the space of trace-class operators $\mathbb{T}$ equipped with the trace norm (see e.g., Lemma S.2.2 in Shen et al., 2020). The latter expectation is understood to be defined in the sense of Bochner integral in the separable Banach space $\left(\mathbb{T},\|\cdot\|_{\text {tr }}\right)$, and for $f, g \in \mathbb{V}$, the outer product operator is by definition $(f \otimes g) h:=\langle h, g\rangle f$. Observe that $\mathcal{C}_{X}(t, t)$ is positive definite, and

$$
\mathcal{C}_{X}(s, t)=\mathcal{C}_{X}^{*}(t, s), \text { for all } s, t \in \mathbb{R}^{d} .
$$

The process $X$ is said to be mean-square or $L^{2}$-continuous if

$$
\mathbb{E}\|X(s)-X(t)\|^{2} \rightarrow 0 \text { as } s \rightarrow t \text {, for all } t \in \mathbb{R}^{d} .
$$

It is easy to see (e.g., Section S.2.2 or Proposition S.2.3 in Shen et al., 2020) that $X$ is mean-square continuous if and only if $\left\|\mathcal{C}_{X}\left(s^{\prime}, t^{\prime}\right)-\mathcal{C}_{X}(t, t)\right\|_{\text {tr }} \rightarrow 0$ as $\left(s^{\prime}, t^{\prime}\right) \rightarrow(t, t)$.

Definition 4.5. A process $X$ is said to be weakly or covariance-stationary if it is second order and its cross covariance is shift invariant, i.e.,

$$
\mathcal{K}_{X}(h):=\mathcal{C}_{X}(s, s+h), \quad \text { for all } h \in \mathbb{R}^{d},
$$

does not depend on $s \in \mathbb{R}^{d}$. The function $\mathcal{K}_{X}(h), h \in \mathbb{R}^{d}$, will be referred to as the stationary covariance function of $X$.

Observe that every stationary covariance function $\mathcal{K}_{X}$ is positive definite in the sense of (4.3) (and hence (4.1)). Also, the $L^{2}$-continuity of a stationary process is equivalent to the continuity of its stationary covariance function at 0 . Thus, the characterization in 
Theorem 4.2 readily holds for the stationary covariance of a stationary process that is $L^{2}$-continuous.

We conclude this section with a version of the classical Cramér stochastic representation of stationary Hilbert-space-valued random fields. Recall that, for $\mathbb{V}=\mathbb{C}$, the well-known integral representation (cf. Cramér, 1942) of a covariance stationary random process $X$ on $\mathbb{R}^{d}$ states that

$$
X(t)=\int_{\mathbb{R}^{d}} e^{\mathrm{i} t^{\top} x} \xi(d x), \quad \text { almost surely, } \quad t \in \mathbb{R}^{d},
$$

where $\xi$ is a second-order random measure with orthogonal increments. To extend this result to a general $\mathbb{V}$, we first have to define integration with respect to a random measure with orthogonal increments in that setting. This is done in Section A.4 of Appendix. Here, we only give the main ideas.

Let $\mathbb{L}^{2}(\Omega)$ be the $L^{2}$ space of all $\mathbb{V}$-valued random elements $\eta$ on the probability space $(\Omega, \mathcal{F}, \mathbb{P})$ with $\mathbb{E}\left[\|\eta\|^{2}\right]<\infty$, equipped with the inner product

$$
\left\langle\eta_{1}, \eta_{2}\right\rangle_{\Omega}:=\mathbb{E}\left\langle\eta_{1}, \eta_{2}\right\rangle .
$$

Definition 4.6. Let $\mu$ be a $\mathbb{T}_{+}$-valued measure on $\mathcal{B}\left(\mathbb{R}^{d}\right)$. A second-order stochastic process $\xi=\left\{\xi(A), A \in \mathcal{B}\left(\mathbb{R}^{d}\right)\right\} \subset \mathbb{L}^{2}(\Omega)$ indexed by the Borel sets is said to be a $\mathbb{V}$-valued orthogonal random measure on $\mathbb{R}^{d}$ with control measure $\mu$ if the following conditions hold:

(i) $\mathbb{E}\left[\left\|\xi\left(A_{n}\right)\right\|^{2}\right] \rightarrow 0$ if $\mu\left(A_{n}\right) \rightarrow 0$,

(ii) $\mu(A \cap B)=\mathbb{E}[\xi(A) \otimes \xi(B)]$, for all $A, B \in \mathcal{B}\left(\mathbb{R}^{d}\right)$, where the expectation is in the sense of Bochner on $\left(\mathbb{T},\|\cdot\|_{\mathrm{tr}}\right)$.

It is straightforward to see that (ii) implies for disjoint $A$ and $B$, that $\xi(A \cup B)=$ $\xi(A)+\xi(B)$, almost surely, so that $\xi$ is in fact an additive set-function. This, combined with the continuity property (i), yields the $\sigma$-additivity of $\xi$ (for more details, see Section A.4).

For a simple function $f(t)=\sum_{i=1}^{n} c_{i} I_{A_{i}}(t)$, with $c_{i} \in \mathbb{C}$ and pairwise disjoint $A_{i}$ 's, we naturally define $\mathcal{I}_{\xi}(f):=\int_{\mathbb{R}^{d}} f(t) \xi(d t):=\sum_{i=1}^{n} c_{i} \xi\left(A_{i}\right)$. Letting $\|\mu\|_{\operatorname{tr}}(A):=\|\mu(A)\|_{\text {tr }}$ be the trace measure of $\mu$, we see that

$$
\mathbb{E}\left[\left\|\mathcal{I}_{\xi}(f)\right\|^{2}\right]=\sum_{i=1}^{n}\left|c_{i}\right|^{2} \mathbb{E}\left\|\xi\left(A_{i}\right)\right\|^{2}=\int_{\mathbb{R}^{d}}|f(t)|^{2}\|\mu\|_{\mathrm{tr}}(d t) .
$$

That is, the linear operator $\mathcal{I}_{\xi}$ is an isometry from the space of simple functions in $L^{2}\left(\mathbb{R}^{d},\|\mu\|_{\text {tr }}\right)$ into the Hilbert space $\mathbb{L}^{2}(\Omega)$. Thus, one can extend the definition of $\mathcal{I}_{\xi}(f)$, by continuity, to all $f \in L^{2}\left(\mathbb{R}^{d},\|\mu\|_{\text {tr }}\right)$. We have moreover that, for all $f, g \in L^{2}\left(\mathbb{R}^{d},\|\mu\|_{\text {tr }}\right)$,

$$
\mathbb{E}\left[\mathcal{I}_{\xi}(f) \otimes \mathcal{I}_{\xi}(g)\right]=\int_{\mathbb{R}^{d}} f(t) \overline{g(t)} \mu(d t),
$$

where the latter integral is in the sense of Bochner (cf. Section A.4 of Appendix).

Theorem 4.7. Let $X=\left\{X(t), t \in \mathbb{R}^{d}\right\}$ be an $L^{2}$-continuous, weakly stationary process taking values in the separable Hilbert space $\mathbb{V}$ and having stationary covariance function $\mathcal{K}$. Then, (4.2) holds and there exists an a.s. unique orthogonal $\mathbb{V}$-valued random measure $\xi$ with control measure $\mu$, such that (4.5) holds.

The proof of this result can be found in Section S.4.3 of Shen et al. (2020). 


\subsection{Spectral theory for general $\operatorname{IRF}_{k}$}

Gel'fand and Vilenkin (1964) provide an illuminating treatment of the spectral theory of generalized stochastic processes, i.e., processes with paths in the space of generalized functions. In this setting, the paths of the stochastic process have derivatives of all orders and one can naturally study processes with stationary $(1+k)$-th order derivatives. One drawback of this treatment is that it is difficult to use generalized process models in practice. Motivated by fundamental problems in spatial statistics, Matheron (1973) developed the framework of intrinsic stationary functions, which allows one to study classical random field models with stationary increments.

In a series of works, Matheron developed the theory of intrinsic random functions, which has become the lingua franca of spatial statistics (see e.g. Chilès and Delfiner, 2012). Our goal here is to extend the Matheron theory to the functional setting, where the underlying stochastic processes take values in a separable Hilbert space $\mathbb{V}$. This is not straightforward and new covariance asymmetry phenomena arise that reflect the potential irreversibility of multivariate IRF's (see Remark 5.21).

Following Matheron (1973), in this section we will focus on second order linear processes $Y=\left\{Y(\lambda), \lambda \in \Lambda_{k}\right\}$, viewed in the weak sense as stochastic processes indexed by $\Lambda_{k}$. That is, $Y$ is a random element in $S\left(\Lambda_{k}, \mathbb{V}\right)$ equipped with the product $\sigma$-field $\mathcal{B}_{\mathbb{V}^{\Lambda_{k}}}$ generated by all finite-dimensional cylinder sets. We emphasize that, in contrast to Section 3, here we no longer require that $Y$ has continuous paths. The (cross) covariance operator of $Y$ is defined as

$$
\mathcal{C}_{Y}(\lambda, \mu):=\mathbb{E}[Y(\lambda) \otimes Y(\mu)], \quad \lambda, \mu \in \Lambda_{k} .
$$

Denote by $\breve{Y}=\left\{\breve{Y}(t), t \in \mathbb{R}^{d}\right\}$ the representation of $Y$ (cf. Section 2.1) in $\breve{S}\left(\Lambda_{k}, \mathbb{V}\right.$ ), i.e., $\breve{Y}(t)=Y\left(\lambda_{t}\right)$, so that

$$
Y(\lambda):=\int \breve{Y}(t) \lambda(d t), \quad \lambda \in \Lambda_{k}
$$

We say that $Y$ is mean-square continuous if $\breve{Y}$ is mean-square continuous in the sense of (4.4).

Definition 4.8. A second order process $\left\{Y(\lambda), \lambda \in \Lambda_{k}\right\} \in S\left(\Lambda_{k}, \mathbb{V}\right)$ is said to be an intrinsic random function of order $k\left(I R F_{k}\right), k=-1,0,1, \ldots$, if

$$
\mathcal{C}_{Y}(\lambda, \mu) \equiv \mathcal{C}_{Y}(w+\lambda, w+\mu), \quad w \in \mathbb{R}^{d}, \lambda, \mu \in \Lambda_{k} .
$$

Note that (4.7) is equivalent to

$$
\mathcal{C}_{Y}(\lambda, \lambda) \equiv \mathcal{C}_{Y}(w+\lambda, w+\lambda), \quad w \in \mathbb{R}^{d}, \lambda \in \Lambda_{k},
$$

by Lemma A.10, and, in turn, to the weak stationarity of $\left\{Y(t+\lambda), t \in \mathbb{R}^{d}\right\}$ in $t$ for all $\lambda \in \Lambda_{k}$. Indeed, if $Y(t+\lambda)$ is stationary in $t$ for all $\lambda$ then (4.8) holds, and if (4.7) holds then $Y(t+\lambda)$ is stationary in $t$ for all $\lambda$.

Definition 4.9. A collection of trace-class operators $\left\{\mathcal{K}(h), h \in \mathbb{R}^{d}\right\} \subset \mathbb{T}$ is said to be conditionally positive definite of degree $k, k=-1,0,1, \ldots$, if for all $n \geq 1$,

$$
\sum_{j=1}^{n} \sum_{j^{\prime}=1}^{n} c_{j} \bar{c}_{j^{\prime}} \mathcal{K}\left(t_{j}-t_{j^{\prime}}\right) \geq 0 \text { (operator positivity) }
$$

for all $c_{i} \in \mathbb{C}, t_{j} \in \mathbb{R}^{d}, 1 \leq i \leq n$, such that $\lambda(d u)=\sum_{j} c_{j} \delta_{t_{j}}(d u) \in \Lambda_{k}$. 
Relation (4.9) can be succinctly written as $\mathcal{K}(\lambda * \widetilde{\lambda}) \geq 0$, where

$$
\widetilde{\lambda}(d u):=\bar{\lambda}(-d u)=\sum_{j} \bar{c}_{j} \delta_{-t_{j}}(d u)
$$

and $\lambda * \mu$ denotes the usual convolution. More generally, with $\lambda=\sum_{j} c_{j} \delta_{t_{j}}$ and $\mu=$ $\sum_{j^{\prime}} d_{j^{\prime}} \delta_{s_{j^{\prime}}} \in \Lambda_{k}$

$$
\mathcal{K}(\lambda * \widetilde{\mu})=\sum_{j} \sum_{j^{\prime}} c_{j} \bar{d}_{j^{\prime}} \mathcal{K}\left(t_{j}-s_{j^{\prime}}\right)
$$

Interestingly, since $(w+\lambda) *(\widetilde{w+\mu}) \equiv \lambda * \widetilde{\mu}$, for all $w \in \mathbb{R}^{d}$, the map $(\lambda, \mu) \mapsto \mathcal{K}(\lambda * \widetilde{\mu})$ is automatically shift invariant. This motivates the following definition.

Definition 4.10. A collection of operators $\mathcal{K}: \mathbb{R}^{d} \rightarrow \mathbb{T}$ is said to be a generalized covariance of $Y$ with degree $k$ if

$$
\mathcal{C}_{Y}(\lambda, \mu)=\mathcal{K}(\lambda * \widetilde{\mu}), \quad \text { for all } \lambda, \mu \in \Lambda_{k} .
$$

Again, by Lemma A.10, (4.11) is equivalent to

$$
\mathcal{C}_{Y}(\lambda, \lambda)=\mathcal{K}(\lambda * \tilde{\lambda}), \quad \text { for all } \lambda \in \Lambda_{k} .
$$

The following result describes the connections between the notions in Definitions 4.84.10 , and gives a spectral representation of a conditionally positive definite $\mathcal{K}$. As a terminology, a polynomial in $\mathbb{T}$ refers to a linear combination of $d$-dimensional monomials with coefficients in $\mathbb{T}$, where the degree is equal to the highest degree of the monomials in the linear combination.

Theorem 4.11. Let $k \geq-1$ and the process $Y=\left\{Y(\lambda), \lambda \in \Lambda_{k}\right\} \in S\left(\Lambda_{k}, \mathbb{V}\right)$ be second order.

(i) If $Y$ has a generalized covariance $\mathcal{K}$ of degree $k$, then $Y$ is $I R F_{k}$ and $\mathcal{K}$ must be conditionally positive definite of degree $k$. Conversely, if $Y$ is a mean-square continuous $I R F_{k}$, then it has a continuous generalized covariance of degree $k$.

(ii) A continuous function $\mathcal{K}: \mathbb{R}^{d} \rightarrow \mathbb{T}$ is conditionally positive definite of degree $k$ if and only if it can be represented as

$$
\mathcal{K}(h)=\int_{\mathbb{R}^{d}} \frac{e^{\mathrm{i} u^{\top} h}-I_{B}(u) P\left(u^{\top} h\right)}{1 \wedge\|u\|^{2 k+2}} \chi(d u)+\mathcal{Q}(h),
$$

where $P(x)=\sum_{j=0}^{2 k+1}(\dot{\mathrm{i}} x)^{j} / j !, B$ is some arbitrary bounded neighborhood of $0, \mathcal{Q}(h)$ is a conditionally positive definite polynomial with degree no more than $2 k+2$ and $\chi$ is a finite $\mathbb{T}_{+}$-valued measure with no point mass at 0 . The measure $\chi$ in (4.12) is unique and does not depend on the choice of the set B. The polynomial $\mathcal{Q}$ therein is unique modulo an additive polynomial of degree $2 k+1$.

The detailed proof of this result can be found in Section S.4.2 of Shen et al. (2020). Note that the proof follows closely the general and elegant treatment of Sasvári (2009).

Remark 4.12. In the notation of Sasvári (2009), our situation corresponds to having a single multiplicative function (character) $\gamma_{1} \equiv 1$ and $y_{1}:=0$ and $k_{1}:=k+1$ and their measure $\sigma$ is our $\left(1 \wedge\|u\|^{2 k+2}\right)^{-1} \chi(d u)$. Observe also that Relation (4.3) in Theorem 4.2 of Sasvári (2009) appears to be missing the non-ignorable degree $2 k+2$ polynomial component in $\mathcal{Q}$ of (4.12). This omission can be attributed to the fact that the spectral measure of a stationary process in the Bochner theorem could have an atom at $\{0\}$, while $\sigma$ and $\chi$ do not. See Section S.4.2 of Shen et al. (2020) for more details. 
The measure $\chi$ and polynomial $\mathcal{Q}$ in (4.12) will be referred to as the spectral characteristics of an $\mathrm{IRF}_{k}$ with generalized covariance $\mathcal{K}$. Note that the spectral characteristics pair $(\chi, \mathcal{Q})$ is unique modulo an additive polynomial of degree $2 k+1$ in the component $\mathcal{Q}$. That is, the generalized covariance in (4.12) is unique up to an additive polynomial of order $2 k+1$. This implies that $\mathcal{K}(\lambda * \widetilde{\mu})$ is uniquely determined for $\lambda, \mu \in \Lambda_{k}$, where $\mathcal{K}(\nu):=\int \mathcal{K}(h) \nu(d h), \nu \in \Lambda$. Notice that $\lambda * \widetilde{\mu} \in \Lambda_{2 k+1}$, for $\lambda, \mu \in \Lambda_{k}$. Thus, in view of (4.11), the covariance structure of an $\mathrm{IRF}_{k}$ process is completely determined by the linear measure-indexed T-valued function $\mathcal{K}(\nu), \nu \in \Lambda_{2 k+1}$. By integrating (4.12) with respect to $\nu \in \Lambda_{2 k+1}$, we obtain

$$
\mathcal{K}(\nu)=\int_{\mathbb{R}^{d}} \frac{\widehat{\nu}(u)}{1 \wedge\|u\|^{2 k+2}} \chi(d u)+\mathcal{Q}(\nu), \nu \in \Lambda_{2 k+1},
$$

where $\widehat{\nu}(u)=\int e^{\mathrm{i} u} u^{\top} x \nu(d x)$ is the Fourier transform of $\nu$. Since $\widehat{\lambda * \widetilde{\mu}}=\hat{\lambda} \bar{\mu}$, the cross covariance operator $\mathcal{C}_{Y}(\lambda, \mu)$ of $Y$ can be uniquely expressed as

$$
\mathcal{C}_{Y}(\lambda, \mu)=\mathcal{K}(\lambda * \widetilde{\mu})=\int_{\mathbb{R}^{d}} \frac{\widehat{\lambda}(u) \overline{\widehat{\mu}(u)}}{1 \wedge\|u\|^{2 k+2}} \chi(d u)+\mathcal{Q}(\lambda * \widetilde{\mu}) .
$$

Now, consider the following counterpart to Definition 4.3.

Definition 4.13. A collection of trace-class operators $\left\{\mathcal{K}(h), h \in \mathbb{R}^{d}\right\} \subset \mathbb{T}$ is said to be conditionally complete positive definite of degree $k, k=-1,0,1, \ldots$, if

$$
\sum_{j=1}^{n} \sum_{j^{\prime}=1}^{n}\left\langle f_{j}, \mathcal{K}\left(\mu_{j} * \widetilde{\mu}_{j^{\prime}}\right) f_{j^{\prime}}\right\rangle \geq 0
$$

for all $f_{j} \in \mathbb{V}, \mu_{j} \in \Lambda_{k}, j=1, \cdots, n$ and $n \in \mathbb{N}$.

Since (4.9) is the special case of (4.15) with $n=1$, conditional complete positive definiteness implies conditional positive definiteness. However, as seen from (4.14), $\mathcal{K}(\lambda * \widetilde{\mu}), \lambda, \mu \in \Lambda_{k}$ is a valid cross-covariance, and hence the operator function $\mathcal{K}$ in (4.12) is conditionally complete positive definite. Thus, Theorem 4.11 implies the following parallel of Corollary 4.4.

Corollary 4.14. If $\mathcal{K}: \mathbb{R}^{d} \rightarrow \mathbb{T}$ is continuous, then Definitions 4.9 and 4.13 are equivalent.

We end this section with a stochastic representation result for continuous $\mathrm{IRF}_{k}$, which parallels the Cramér representation in Theorem 4.7. The proof is given in Section S.4.4 in Shen et al. (2020)

Theorem 4.15. Let $k \geq-1$ and the process $Y=\left\{Y(\lambda), \lambda \in \Lambda_{k}\right\}$ in $S\left(\Lambda_{k}, \mathbb{V}\right)$ be meansquare continuous. Then $Y$ is $I R F_{k}$ if and only if it can be uniquely represented as

$$
Y(\lambda)=\sum_{\left(j_{1}, \ldots, j_{d}\right) \in J} \frac{\partial^{k+1} \widehat{\lambda}}{\partial^{j_{1}} \cdots \partial^{j_{d}}}(0) \cdot Z_{j_{1} \cdots j_{d}}+\int_{\mathbb{R}^{d}} \frac{\widehat{\lambda}(u)}{1 \wedge\|u\|^{k+1}} \xi(d u) \quad \text { a.s. }
$$

for all $\lambda \in \Lambda_{k}$, where

(i) $J=\left\{\left(j_{1}, \ldots, j_{d}\right): j_{1}, \ldots, j_{d} \geq 0\right.$ and $\left.j_{1}+\cdots+j_{d}=k+1\right\}$,

(ii) $\xi$ is an a.s. unique random orthogonal measure $\xi$ on $\left(\mathbb{R}^{d}, \mathcal{B}\left(\mathbb{R}^{d}\right)\right)$ with control measure $\chi$, where $\chi$ is a finite $\mathbb{T}_{+}$-valued measure with no point mass at 0 , and

(iii) the $Z_{j_{1} \cdots j_{d}}$ are uncorrelated random variables with values in $\mathbb{V}$ and are uncorrelated with $\xi$.

Remark 4.16. Berschneider (2012) also obtains the stochastic representation of $\operatorname{IRF}_{k}$ with the more abstract setting of locally compact Abelian domains. Our result here can be considered as an extension to the case of $\mathbb{V}$-valued processes connecting $\xi(d x)$ with the covariance operator functions in Theorem 4.11 explicitly. 


\subsection{Real and complex $\operatorname{IRF}_{k}$ 's: Covariance (ir)reversibility}

The general treatment in the previous two subsections involves an abstract separable Hilbert space $\mathbb{V}$ over the field of complex numbers $\mathbb{C}$. In practice, however, one often deals with Hilbert spaces of real-valued functions and it is useful to know how our results specialize to this setting. Furthermore, the distribution of a zero-mean Gaussian process taking values in a complex Hilbert space $\mathbb{V}$ cannot be directly characterized using their covariance structure, alone. To this end one needs to consider both real and complex Hilbert spaces.

Real and imaginary parts in a complex Hilbert space. In an abstract complex Hilbert space $\mathbb{V}$ the notions of a real and imaginary part of an element $z(\in \mathbb{V})$ are not well defined unless one fixes a basis. Let $\mathcal{E}:=\left\{e_{j}, j \in \mathbb{N}\right\}$ be a fixed CONS of $\mathbb{V}$. Then one can postulate that the CONS $\mathcal{E}$ is real and for each $z=\sum_{j} z_{j} e_{j} \in \mathbb{V}$, with coordinates $z_{j}:=\left\langle z, e_{j}\right\rangle$, we can define

$$
\Re(z) \equiv \Re_{\mathcal{E}}(z):=\sum_{j} \Re\left(z_{j}\right) e_{j} \quad \text { and } \quad \Im(z) \equiv \Im_{\mathcal{E}}(z):=\sum_{j} \Im\left(z_{j}\right) e_{j},
$$

as the real and imaginary parts of $z$, relative to the CONS $\mathcal{E}$. (Should one change the basis $\mathcal{E}$ the notions of real and imaginary part may change.) Notice that $\mathbb{V}_{\mathbb{R}}:=\{z \in \mathbb{V}$ : $\Im(z)=0\}$ is invariant to addition and multiplication by real scalars and it becomes a real Hilbert space, with the inner product inherited from $\mathbb{V}$. All elements of $\mathbb{V}$ that belong to $\mathbb{V}_{\mathbb{R}}$ will be referred to as real.

For $z \in \mathbb{V}$, we shall write $z=\Re(z)+\dot{i} \Im(z)$ and naturally define the complex conjugate $\bar{z}:=\Re(z)-\mathrm{i} \Im(z)$. The complex conjugate operation as well as the real and imaginary part operators extend to $\mathbb{V}$-valued random elements in a straightforward manner and we shall say that $x \in \mathbb{V}$ is real if $x \in \mathbb{V}_{\mathbb{R}}$, i.e., if its imaginary part is zero.

The complex conjugate of a linear operator $\mathcal{A}: \mathbb{V} \rightarrow \mathbb{V}$ is defined as: $\overline{\mathcal{A}}(x):=$ $\overline{\mathcal{A}(\bar{x})}, \quad x \in \mathbb{V}$. This implies that $\overline{\mathcal{A}(x)}=\overline{\mathcal{A}}(\bar{x})$, the operator $\overline{\mathcal{A}}$ is also linear and one can define the real and imaginary parts of $\mathcal{A}$ in as: $\Re(\mathcal{A}):=(\mathcal{A}+\overline{\mathcal{A}}) / 2$ and $\Im(\mathcal{A}):=$ $(\mathcal{A}-\overline{\mathcal{A}}) / 2$ i. Thus, $\mathcal{A}=\Re(\mathcal{A})+\dot{i} \Im(\mathcal{A})$ and the usual operations with complex numbers and vectors extend to the operator Banach algebra over the complex Hilbert space $\mathbb{V}$. Note that the real and imaginary parts of $\mathcal{A}$ can be equivalently defined in terms of the real and imaginary parts of the coordinates of $\mathcal{A}$ in the fixed CONS $\mathcal{E}$. We shall say that an operator $\mathcal{A}$ is real if $\mathcal{A}=\Re(\mathcal{A})$.

Real $I R F_{k}$ 's. The above discussion shows how one can specialize and interpret the results in Sections 4.1 and 4.2 for the case of real Hilbert spaces $\mathbb{V}_{\mathbb{R}}$. Indeed, let $\Lambda_{k}(\mathbb{R})$ be the set of all real $\lambda \in \Lambda_{k}$. It is easy to see that $\Lambda_{k}=\Lambda_{k}(\mathbb{R})+\dot{i} \Lambda_{k}(\mathbb{R})$.

Suppose now that $Y$ is a $\mathbb{V}_{\mathbb{R}}$-valued $\mathrm{IRF}_{k}$. That is, Definition 4.8 holds with $\Lambda_{k}$ replaced by $\Lambda_{k}(\mathbb{R})$. Then, by linearity, $Y$ extends uniquely to a $\mathbb{V}$-valued $\operatorname{IRF}_{k}$ as follows

$$
Y(\lambda):=Y(\Re(\lambda))+\dot{\mathrm{I}} Y(\Im(\lambda)), \lambda \in \Lambda_{k},
$$

where in fact $Y(\Re(\lambda))$ and $Y(\Im(\lambda))$ are real (belong to $\mathbb{V}_{\mathbb{R}}$ ). This leads us to the following

Definition 4.17. $A \mathbb{V}$-valued $I_{R} F_{k} Y$ is said to be real if $Y(\lambda)$ is real for all $\lambda \in \Lambda_{k}(\mathbb{R})$.

Thus, there is a one-to-one correspondence between the real $\operatorname{IRF}_{k}$ 's in $\mathbb{V}$ and the $\mathbb{V}_{\mathbb{R}}$-valued $\mathrm{IRF}_{k}$ 's as processes indexed by $\Lambda_{k}(\mathbb{R})$.

Proposition 4.18. Let $Y$ be a mean-square continuous $I R F_{k}$ taking values in $\mathbb{V}$ and having spectral characteristics $(\chi, \mathcal{Q})$.

(i) $Y$ is real if and only if in Relation (4.16) the vectors (i) ${ }^{k+1} Z_{j_{1} \cdots j_{d}}$ are real and the orthogonal measure $\xi$ is Hermitian, i.e., $\xi(-A)=\overline{\xi(A)}$, almost surely, for all $A \in \mathcal{B}\left(\mathbb{R}^{d} \backslash\{0\}\right)$. 
Tangent fields, intrinsic stationarity, and self similarity

(ii) If $Y$ is real, then $\mathcal{Q}$ has real (operator) coefficients (modulo polynomials of degree up to $2 k+1$ ) and the spectral measure $\chi$ is Hermitian, i.e., $\overline{\chi(-A)}=\chi(A)$, for all $A \in \mathcal{B}\left(\mathbb{R}^{d} \backslash\{0\}\right)$.

(iii) Conversely, if $\chi$ is Hermitian and $\mathcal{Q}$ real, then there is a real $I_{R F} Y$ with spectral characteristics $(\chi, \mathcal{Q})$. Let now $Y$ and $Y^{\prime}$ be two real $I R F_{k}$ with the same spectral characteristics $(\chi, \mathcal{Q})$ and such that $\mathbb{E}\left[Y(\lambda) \otimes Y^{\prime}(\mu)\right]=\mathbb{E}\left[Y^{\prime}(\lambda) \otimes Y(\mu)\right], \lambda, \mu \in \Lambda_{k}$. Then, for any $a, b \in \mathbb{R}$, with $a^{2}+b^{2}=1$, the $\operatorname{IRF}_{k}$ defined as $\widetilde{Y}(\lambda)=a Y(\lambda)+\dot{\mathrm{i}} b Y^{\prime}(\lambda)$ has the same spectral characteristics as $Y$ and $Y^{\prime}$.

The proof is given in Section A.4 of the Appendix.

Covariance (ir)reversibility. Next, we comment on an important covariance irreversibility phenomenon, which arises in the case of vector valued processes. It extends the notion of time reversibility for vector time series.

Definition 4.19. We shall say that an $I R F_{k} Y$ with generalized operator covariance $\mathcal{K}(\cdot)$ is covariance-symmetric or -reversible if

$$
\mathcal{K}(\nu)=\mathcal{K}((-1) \cdot \nu), \quad \text { for all } \nu \in \Lambda_{2 k+1} .
$$

Observe that the symmetry of the generalized covariance is equivalent to the fact that the $\operatorname{IRF}_{k}$ processes $\{Y((-1) \cdot \lambda)\}$ and $\{Y(\lambda)\}$ have the same covariance structure. Indeed, recall that

$$
\mathbb{E}[Y(\lambda) \otimes Y(\mu)]=\mathcal{C}_{Y}(\lambda, \mu)=\mathcal{K}(\lambda * \widetilde{\mu}), \lambda, \mu \in \Lambda_{k}
$$

and observe that $(-1) \cdot \lambda * \widetilde{\mu}=((-1) \cdot \lambda) *(\widetilde{(-1) \cdot \mu})$. Thus, the $\operatorname{IRF}_{k}$ process $\tilde{Y}(\lambda):=$ $Y((-1) \cdot \lambda)$ has covariance $\mathcal{K}((-1) \cdot \nu)$.

In the simple case, where $Y$ takes real scalar values, all $\mathrm{IRF}_{k}$ 's are automatically covariance-symmetric. This is perhaps why symmetry is often taken for granted. In the multivariate and especially function-valued case, however, covariance-symmetry is an exception rather than a rule. Naturally, in view of (4.13) and the uniqueness of the spectral measure, (4.19) holds if and only if $\chi(-A)=\chi(A)$, for all $A \in \mathcal{B}\left(\mathbb{R}^{d} \backslash\{0\}\right)$. This simple observation and Proposition 4.18 yield the following fact (see also Theorem 5.1 in Didier and Pipiras, 2011).

Proposition 4.20. A real mean-square continuous $I R F_{k} Y$ is covariance symmetric, if and only if its spectral measure $\chi$ is real.

We end this section with a comment on the use of the results from Sections 4.1 and 4.2 in the context of Gaussian processes. Recall that a $\mathbb{V}$-valued random element $Y$ is said to be Gaussian, if $\langle Y, f\rangle$ is a complex Gaussian variable, for each $f \in \mathbb{V}$. This means that the joint distribution of $(\Re(\langle Y, f\rangle), \Im(\langle Y, f\rangle))$ is bivariate normal, for all $f \in \mathbb{V}$. Equivalently, $Y$ is Gaussian in $\mathbb{V}$ if and only if $\widetilde{Y}:=(\Re(Y), \Im(Y))$ is a Gaussian element in the real Hilbert space $\mathbb{V}_{\mathbb{R}}^{2}:=\mathbb{V}_{\mathbb{R}} \times \mathbb{V}_{\mathbb{R}}$.

Remark 4.21 (Characterization of Gaussian $\mathrm{IRF}_{k}$ 's). Part (iii) of Proposition 4.18 is a manifestation of the fact that the covariance structure alone does not determine the distribution of zero-mean Gaussian processes taking values in complex Hilbert spaces (see also Example S.5.1 in Shen et al., 2020). To determine the distribution of a zero-mean Gaussian $\operatorname{IRF}_{k} Y=\left\{Y(\lambda), \lambda \in \Lambda_{k}\right\}$, one needs to know both the crosscovariance and pseudo cross-covariance operators: $\mathcal{C}_{Y}(\lambda, \mu)=\mathbb{E}[Y(\lambda) \otimes Y(\mu)]$ and $\mathcal{C}_{Y, \bar{Y}}(\lambda, \mu)=\mathbb{E}[Y(\lambda) \otimes \bar{Y}(\mu)]$ (see e.g., Section S.5.1 and Corollary S.5.3 in Shen et al., 2020).

Equivalently, the distribution of a $\mathbb{V}$-valued Gaussian $\mathrm{IRF}_{k}$ is completely determined by the real $\operatorname{IRF}_{k} \tilde{Y}(\cdot):=(\Re(Y)(\cdot), \Im(Y)(\cdot))$ in the product space $\mathbb{V}^{2}$. Since the law of the real Gaussian $\operatorname{IRF}_{k} \widetilde{Y}$ is determined by its cross-covariance, the results of Sections 4.1 and 4.2 provide a complete characterization of the $\mathbb{V}$-valued Gaussian $\operatorname{IRF}_{k}$ 's. 


\section{Second-order covariance self-similar IRF $_{k}$ 's}

In view of Theorems 3.4 and 3.8, essentially all tangent fields are self-similar $\operatorname{IRF}_{k}$. This motivates a more in-depth study of self-similar $\mathrm{IRF}_{k}$ 's. In this section, we focus on second order covariance self-similar $\mathrm{IRF}_{k}$ 's with respect to linear operator-scaling actions. We establish their covariance structure and spectral representation. Section 5.1 addresses the general case, Section 5.2 discusses examples and related work, while Section 5.3 deals with the scalar scaling action, where the generalized covariance can be written in closed form.

\subsection{Linear operator scaling}

Let $\mathcal{H}: \mathbb{V} \rightarrow \mathbb{V}$ be a bounded linear operator on the Hilbert space $\mathbb{V}$. Consider the operator scaling actions $T_{c}:=c^{\mathcal{H}}, c>0$, where $c^{\mathcal{H}}$ is interpreted as $\exp \{\log (c) \mathcal{H}\}$ and as usual,

$$
e^{\mathcal{H}}:=\sum_{n=0}^{\infty} \frac{\mathcal{H}^{n}}{n !}
$$

The latter series converges in operator norm and $\left\|e^{\mathcal{H}}\right\|_{\mathrm{op}} \leq e^{\|\mathcal{H}\|_{\mathrm{op}}}$. We have moreover that if the bounded operators $\mathcal{H}_{1}$ and $\mathcal{H}_{2}$ commute, i.e., $\mathcal{H}_{1} \mathcal{H}_{2}=\mathcal{H}_{2} \mathcal{H}_{1}$, then $e^{\mathcal{H}_{1}} e^{\mathcal{H}_{2}}=$ $e^{\mathcal{H}_{1}+\mathcal{H}_{2}}=e^{\mathcal{H}_{2}} e^{\mathcal{H}_{1}}$. This readily implies that $e^{\mathcal{H}}$ has a bounded inverse $\left(e^{\mathcal{H}}\right)^{-1}=e^{-\mathcal{H}}$. Consequently, $c^{\mathcal{H}}, c>0$ is a strongly (operator) continuous and invertible group action on $\mathbb{V}$, i.e., $c_{1}^{\mathcal{H}} c_{2}^{\mathcal{H}}=\left(c_{1} c_{2}\right)^{\mathcal{H}}, c_{1}, c_{2}>0$. In fact, using the power-series representation (5.1), one can readily show that $c \mapsto c^{\mathcal{H}}$ is continuously Fréchet differentiable with derivative $c^{\mathcal{H}-1} \mathcal{H}$, i.e.,

$$
\left\|\frac{1}{h}\left((c+h)^{\mathcal{H}}-c^{\mathcal{H}}\right)-c^{\mathcal{H}-1} \mathcal{H}\right\|_{\mathrm{op}} \rightarrow 0, \quad \text { as } h \rightarrow 0,
$$

where here and below $\mathcal{H}-a, a \in \mathbb{R}$ is interpreted as $\mathcal{H}-a \mathrm{I}$, so that $c^{\mathcal{H}-1}=c^{-1} c^{\mathcal{H}}$.

As in Definition 3.2, we consider the following notion of covariance operator selfsimilarity.

Definition 5.1. Fix an arbitrary bounded linear operator $\mathcal{H}$ on $\mathbb{V}$. A second order $I R F_{k}$ $Y$ is said to be covariance $\mathcal{H}$-self-similar, if $\left\{Y(c \cdot \lambda), \lambda \in \Lambda_{k}\right\}$ and $\left\{c^{\mathcal{H}} Y(\lambda), \lambda \in \Lambda_{k}\right\}$ have the same operator cross-covariance function for all $c>0$.

Remark 5.2. If the $\operatorname{IRF}_{k}$ process $Y$ is real and Gaussian, then $Y$ is covariance $\mathcal{H}$-selfsimilar if and only if it is $\mathcal{H}$-self-similar in the following stronger sense:

$$
\left\{Y(c \cdot \lambda), \lambda \in \Lambda_{k}\right\} \stackrel{f d d}{=}\left\{c^{\mathcal{H}} Y(\lambda), \lambda \in \Lambda_{k}\right\}, \text { for all } c>0 .
$$

We emphasize that $\mathcal{H}$ in Definition 5.1 and (5.3) is an arbitrary bounded linear operator and we do not require that $c \mapsto c^{\mathcal{H}}$ be a scaling action on $\mathbb{V}$ in the sense of Definition 2.7 (see also Remark 2.9). If $T_{c}:=c^{\mathcal{H}}$ is a scaling action, however, then (5.3) recovers the notion of self-similarity in Definition 3.2.

Let now $Y$ be a second order, mean-square continuous $\operatorname{IRF}_{k}$ with operator autocovariance $\mathcal{K}$ and spectral characteristics $(\chi, \mathcal{Q})$. By Theorem 4.15, we have the decomposition

$$
Y(\lambda)=Y_{(0, \mathcal{Q})}(\lambda)+Y_{(\chi, 0)}(\lambda), \text { almost surely, }
$$

for all $\lambda \in \Lambda_{k}$, where $\left\{Y_{(0, \mathcal{Q})}(\lambda)\right\}$ and $\left\{Y_{(\chi, 0)}(\lambda)\right\}$ are orthogonal mean-square continuous $\operatorname{IRF}_{k}$ 's with spectral characteristics $(0, \mathcal{Q})$ and $(\chi, 0)$, respectively. This decomposition is second order unique. Therefore, it follows that $Y$ is covariance $\mathcal{H}$-self-similar if and only 
if both the components $Y_{(0, \mathcal{Q})}$ and $Y_{(\chi, 0)}$ are covariance $\mathcal{H}$-self-similar. More precisely, we have the following general characterization of covariance $\mathcal{H}$-self-similar $\mathrm{IRF}_{k}$ 's. For convenience, write

$$
\chi_{k}(d x)=\frac{\chi(d x)}{1 \wedge\|x\|^{2 k+2}} .
$$

Theorem 5.3. Let $Y$ be an $L^{2}$-continuous $I R F_{k}$ with spectral characteristics $(\chi, \mathcal{Q})$. Let also $\mathcal{H}$ be a bounded linear operator.

(i) We have that $Y$ is covariance $\mathcal{H}$-self-similar if and only if for all $c>0$ and $\lambda, \mu \in \Lambda_{k}$

$$
\chi_{k}(d x)=c^{-\mathcal{H}} \chi_{k}(d x / c) c^{-\mathcal{H}^{*}} \quad \text { and } \quad \mathcal{Q}(\lambda * \widetilde{\mu})=c^{k+1-\mathcal{H}} \mathcal{Q}(\lambda * \widetilde{\mu}) c^{k+1-\mathcal{H}^{*}},
$$

where $\chi_{k}$ is as in (5.5).

Suppose henceforth that (5.6) holds and consider the polar coordinates $(r, \theta):=$ $(\|x\|, x /\|x\|)$ in $\mathbb{R}^{d} \backslash\{0\}$.

(ii) There exists a finite $\mathbb{T}_{+}$-valued measure $\sigma$ on the unit sphere $\mathbb{S}=\{\|x\|=1\}$ such that

$$
\chi_{k}(D)=\int_{0}^{\infty} r^{-\mathcal{H}}\left(\int_{\mathbb{S}} 1_{D}(r \theta) \sigma(d \theta)\right) r^{-\mathcal{H}^{*}} \frac{d r}{r},
$$

for all Borel sets $D \in \mathcal{B}\left(\mathbb{R}^{d} \backslash\{0\}\right)$ that are bounded away from 0. If (5.7) holds, we simply write

$$
\chi_{k}(d r d \theta)=r^{-\mathcal{H}} \sigma(d \theta) r^{-\mathcal{H}^{*}} \frac{d r}{r}
$$

and refer to (5.8) as a disintegration formula for $\chi_{k}$.

(iii) The measure $\sigma$ in (5.8) is uniquely determined by the measure $\chi_{k}$ and it does not depend on the possibly non-unique operator $\mathcal{H}$ in (5.6).

(iv) The component $Y_{(\chi, 0)}$ of $Y$ admits the Cramér-type stochastic integral representation

$$
Y_{(\chi, 0)}(\lambda)=\int_{0}^{\infty} \int_{\mathbb{S}} \hat{\lambda}(r \theta) W(d r, d \theta), \quad \text { almost surely, }
$$

$\lambda \in \Lambda_{k}$, where $W(d r, d \theta)$ is an orthogonal $\mathbb{V}$-valued random measure on $(0, \infty) \times \mathbb{S}$, such that

$$
\mathbb{E}[W(d r, d \theta) \otimes W(d r, d \theta)]=r^{-(\mathcal{H}+1 / 2)} \sigma(d \theta) r^{-\left(\mathcal{H}^{*}+1 / 2\right)} d r .
$$

The proof of this result is deferred to Section A.5, below.

Remark 5.4 (The support of an $\mathcal{H}$-self-similar $\mathrm{IRF}_{k}$ is $\mathcal{H}$-invariant). The self-similarity exponent operator $\mathcal{H}$ can in principle be arbitrary outside the support of the $\operatorname{IRF}_{k}$ process $Y$. The support of $Y$, denoted $\operatorname{supp}(Y)$, is the smallest closed linear subspace of $\mathbb{V}$, which contains all $Y(\lambda)$ 's almost surely. One can show that $\mathcal{H}(\operatorname{supp}(Y))$ is a dense subset of $\operatorname{supp}(Y)$. This allows one to essentially restrict the operator $\mathcal{H}$ to $\operatorname{supp}(Y)$ (see Section S.6 in Shen et al., 2020, for more details).

Remark 5.5. If $Y$ is $\mathcal{H}$-self-similar, so are its components $Y_{(\chi, 0)}$ and $Y_{(0, \mathcal{Q})}$ in (5.4). While this decomposition is unique in law, the operator $\mathcal{H}$ need not be unique. See for example Didier et al. (2017) and Remark 5.14 below. For example, the polynomial component $Y_{(0, \mathcal{Q})}$ is always $(k+1) \cdot$ I-self-similar. In general, however, we cannot conclude that $\mathcal{H}=(k+1) \cdot \mathrm{I}$, where I is the identity. The non-uniqueness of the operator self-similarity exponent in the general setting of $\mathrm{V}$-valued $\mathrm{IRF}_{k}$ 's is an interesting problem of future research. 
For simplicity, in the rest of this section we suppose that $Y \stackrel{d}{=} Y_{(\chi, 0)}$ has a trivial polynomial component $Y_{(0, \mathcal{Q})}=0$. We will examine two classes of operators $\mathcal{H}$ which can serve as self-similarity exponents of $Y$. We begin with a simple criterion.

Corollary 5.6. A finite $\mathbb{T}_{+}$-valued measure $\chi$ is the spectral measure of an $L^{2}$-continuous, covariance $\mathcal{H}$-self-similar $I R F_{k}$ if and only if for some finite $\mathbb{T}_{+}$-valued measure $\sigma$ on $\mathbb{S}$, we have

$$
\chi_{k}(d x) \equiv \frac{1}{1 \wedge\|x\|^{2 k+2}} \chi(d x)=r^{-\mathcal{H}} \sigma(d \theta) r^{-\mathcal{H}^{*}} r^{-1} d r
$$

and

$$
\int_{0}^{\infty} r^{-1}\left(1 \wedge r^{2 k+2}\right) \operatorname{trace}\left(r^{-\mathcal{H}} \sigma(\mathbb{S}) r^{-\mathcal{H}^{*}}\right) d r<\infty
$$

where $(r, \theta):=(\|x\|, x /\|x\|)$ are the polar coordinates in $\mathbb{R}^{d} \backslash\{0\}$.

Proof. 'only if': Let $Y$ be a continuous covariance $\mathcal{H}$-self-similar $\mathrm{IRF}_{k}$. Then, by Theorem 5.3, $\chi_{k}$ satisfies the disintegration formula in (5.11). We have moreover that

$$
\chi\left(\mathbb{R}^{d}\right)=\int_{0}^{\infty} r^{-1}\left(1 \wedge r^{2 k+2}\right) r^{-\mathcal{H}} \sigma(\mathbb{S}) r^{-\mathcal{H}^{*}} d r \in \mathbb{T}_{+} .
$$

Since $\chi\left(\mathbb{R}^{d}\right)$ is self-adjoint and positive definite, $\left\|\chi\left(\mathbb{R}^{d}\right)\right\|_{\operatorname{tr}}=\operatorname{trace}\left(\chi\left(\mathbb{R}^{d}\right)\right)<\infty$, which proves (5.12).

'if': Conversely, suppose that $\sigma$ is a finite $\mathbb{T}_{+}$-valued measure on $\mathbb{S}$ such that (5.12) holds. Then, the fact that for all $B \in \mathcal{B}(\mathbb{S})$ and $r>0, r^{-\mathcal{H}} \sigma(B) r^{-\mathcal{H}^{*}} \leq r^{-\mathcal{H}} \sigma(\mathbb{S}) r^{-\mathcal{H}^{*}}$ as positive operators in $\mathrm{T}_{+}$, implies that

$$
\chi(D):=\int_{0}^{\infty} r^{-1}\left(1 \wedge r^{2 k+2}\right) r^{-\mathcal{H}} \int_{\mathbb{S}} 1_{D}(r \theta) \sigma(d \theta) r^{-\mathcal{H}^{*}} d r, \quad D \in \mathcal{B}\left(\mathbb{R}^{d} \backslash\{0\}\right)
$$

is well-defined in the sense of Bochner. The so-defined $\chi$ is a finite $\mathbb{T}_{+}$-valued Borel measure on $\mathbb{R}^{d} \backslash\{0\}$, which can be taken as the spectral measure of an $\mathrm{IRF}_{k}$ process $Y=Y_{(\chi, 0)}$ with trivial polynomial component. Clearly, $\chi_{k}$ defined as in (5.11) satisfies the scaling property (5.6), which entails the covariance $\mathcal{H}$-self-similarity of $Y$.

- Normal diagonalizable exponents. Corollary 5.6 allows us to provide a complete characterization of the valid pairs $(\mathcal{H}, \sigma)$ of operator exponents and spectral measures in the important case where $\mathcal{H}$ is normal and diagonalizable operator. Namely, suppose $\mathcal{H}$ is a normal operator with

$$
\mathcal{H}=\sum_{j=1}^{\infty} \lambda_{j} e_{j} \otimes e_{j},
$$

where $\lambda_{j} \in \mathbb{C}$ and where $\left\{e_{j}\right\}$ is a CONS of Range $(\mathcal{H})=\operatorname{Range}\left(\mathcal{H}^{*}\right)$. The convergence of the last series is understood in the weak operator topology.

Theorem 5.7. Let $\mathcal{H}$ be a normal diagonalizable operator as in (5.13) and let $\sigma$ be a finite $\mathbb{T}_{+}$-valued measure on $\mathbb{S}$. The measure $\chi(d r d \theta)=\left(1 \wedge r^{2 k+2}\right) r^{-\mathcal{H}} \sigma(d \theta) r^{-\mathcal{H}^{*}} r^{-1} d r$ is the spectral measure of an $\mathcal{H}$-self-similar $I R F_{k}$, if and only if

$$
0<\Re\left(\lambda_{j}\right)<k+1, \quad \text { whenever }\left\langle\sigma(\mathbb{S}) e_{j}, e_{j}\right\rangle>0
$$

and

$$
\sum_{j}\left(\frac{1}{k+1-\mathfrak{R}\left(\lambda_{j}\right)}+\frac{1}{\mathfrak{R}\left(\lambda_{j}\right)}\right)\left\langle\sigma(\mathbb{S}) e_{j}, e_{j}\right\rangle<\infty
$$


Proof. First, we prove the 'if' part. Let $\sigma \in \mathbb{T}_{+}$satisfy (5.14) and define (in polar coordinates) $\chi(d r d \theta)=\left(1 \wedge r^{2 k+2}\right) r^{-\mathcal{H}} \sigma(d \theta) r^{-\mathcal{H}^{*}} r^{-1} d r$. By the simple fact

$$
\operatorname{trace}\left(r^{-\mathcal{H}} \sigma(\mathbb{S}) r^{-\mathcal{H}^{*}}\right)=\sum_{j} r^{-2 \mathfrak{R}\left(\lambda_{j}\right)}\left\langle\sigma(\mathbb{S}) e_{j}, e_{j}\right\rangle,
$$

we obtain

$$
\begin{aligned}
& \int_{0}^{\infty} r^{-1}\left(1 \wedge r^{2 k+2}\right) \operatorname{trace}\left(r^{-\mathcal{H}} \sigma(\mathbb{S}) r^{-\mathcal{H}^{*}}\right) d r \\
& =\sum_{j}\left\langle\sigma(\mathbb{S}) e_{j}, e_{j}\right\rangle \int_{0}^{\infty}\left(1 \wedge r^{2 k+2}\right) r^{-2 \mathfrak{R}\left(\lambda_{j}\right)-1} d r \\
& =\frac{1}{2} \sum_{j}\left(\frac{1}{k+1-\mathfrak{R}\left(\lambda_{j}\right)}+\frac{1}{\mathfrak{R}\left(\lambda_{j}\right)}\right)\left\langle\sigma(\mathbb{S}) e_{j}, e_{j}\right\rangle<\infty
\end{aligned}
$$

where the integration is justified by (5.14) and (5.15). By Corollary 5.6, $\chi$ is the spectral measure of a covariance $\mathcal{H}$-self-similar $\operatorname{IRF}_{k}$.

Conversely, suppose $\chi$ is the spectral measure of a covariance $\mathcal{H}$-self-similar $\mathrm{IRF}_{k}$. In order for the integral in (5.12) to be finite, the calculations in (5.16) show that both (5.14) and (5.15) must hold.

- General bounded operator exponents. Suppose now that $\mathcal{H}$ is a general bounded operator, which need not be normal nor diagonalizable. In this case, we cannot provide a complete characterization of the covariance $\mathcal{H}$-self-similar $\mathrm{IRF}_{k}$ 's, but still furnish a general sufficient condition using Riesz functional calculus (see e.g., Ch. VII.4 in Conway, 2007). Recall that the spectrum $\operatorname{sp}(\mathcal{H})$ of a bounded operator consists of all $z \in \mathbb{C}$ such that $(\mathcal{H}-z \cdot \mathrm{I})$ has no bounded inverse. The $\operatorname{spectrum} \operatorname{sp}(\mathcal{H})$ is always a non-empty compact subset of $\mathbb{C}$ and $\operatorname{sp}\left(\mathcal{H}^{*}\right)=\{\bar{z}: z \in \operatorname{sp}(\mathcal{H})\}$ consists of the complex conjugates of the elements in the spectrum of $\mathcal{H}$. If $\Gamma$ is a rectifiable curve containing $\operatorname{sp}(\mathcal{H})$ in its interior then for every holomorphic function $f$ on an open set containing the curve $\Gamma$ along with its interior, we define

$$
f(\mathcal{H}):=\frac{1}{2 \pi \dot{\mathrm{i}}} \oint_{\Gamma} \frac{f(z)}{z-\mathcal{H}} d z,
$$

where the latter integral over $\Gamma$ is considered in the positive direction and $1 /(z-\mathcal{H}):=$ $(z \cdot \mathrm{I}-\mathcal{H})^{-1}$ is a bounded operator since $z \in \Gamma \subset \mathbb{C} \backslash \operatorname{sp}(\mathcal{H})$. Since $f(z)=\exp \{-\log (r) z\}$ is analytic for all $r>0$, we can use the above Riesz functional calculus tool to study the operator $r^{-\mathcal{H}}$.

Proposition 5.8. Let $\mathcal{H}$ be a bounded operator and let $\Re(\operatorname{sp}(\mathcal{H}))$ denote the set of real parts of its spectrum. If

$$
\Re(\operatorname{sp}(\mathcal{H})) \subset(0, k+1),
$$

for some $k \geq 0, k \in \mathbb{Z}$, then for all finite $\mathbb{T}_{+}$-valued measures $\sigma$ on $\mathbb{S}$, we have that

$$
\chi(d r d \theta):=\left(1 \wedge r^{2 k+2}\right) r^{-\mathcal{H}} \sigma(d \theta) r^{-\mathcal{H}^{*}} r^{-1} d r,
$$

is the spectral measure of a covariance $\mathcal{H}$-self-similar IRF $F_{k}$.

Proof. We will show first that, for some $\epsilon>0$ and $C_{\mathcal{H}}>0$,

$$
\left\|r^{-\mathcal{H}}\right\|_{\mathrm{op}} \leq C_{\mathcal{H}} \cdot\left(r^{-(k+1)+\epsilon} 1_{(0,1)}(r)+r^{-\epsilon} 1_{[1, \infty)}(r)\right) .
$$


Recall that $\operatorname{sp}(\mathcal{H})$ is a compact subset of $\mathbb{C}$. This fact and the assumption (5.18) on the spectrum of $\mathcal{H}$ implies that $\Re(\operatorname{sp}(\mathcal{H})) \subset(\epsilon, k+1-\epsilon)$, for some $\epsilon>0$. Since $\operatorname{sp}(\mathcal{H})$ is compact, one can take a closed curve $\Gamma$ containing $\operatorname{sp}(\mathcal{H})$ in its interior, such that $\epsilon \leq \Re(z) \leq k+1-\epsilon$ for all $z \in \Gamma$. Note that $z \mapsto\left\|(z \cdot \mathrm{I}-\mathcal{H})^{-1}\right\|_{\text {op }}$ is a continuous function of $z$ for all $z \in \Gamma \subset \mathbb{C} \backslash \operatorname{sp}(\mathcal{H})$. Thus, since $\Gamma$ is a compact set, we have that

$$
\max _{z \in \Gamma}\left\|(z \cdot \mathrm{I}-\mathcal{H})^{-1}\right\|_{\mathrm{op}}=: C_{\mathcal{H}}(\Gamma)<\infty .
$$

Now, by applying (5.17) to $f(z):=r^{-\mathcal{H}}=e^{-\log (r) \mathcal{H}}$, for all $r>0$, we obtain

$$
\begin{aligned}
\left\|r^{-\mathcal{H}}\right\|_{\mathrm{op}} & \leq \frac{1}{2 \pi} \int_{\Gamma}\left|e^{-\log (r) z}\right|\left\|(z \cdot \mathrm{I}-\mathcal{H})^{-1}\right\|_{\mathrm{op}}|d z| \\
& \leq \frac{C_{\mathcal{H}}(\Gamma)}{2 \pi} \operatorname{Len}(\Gamma) \sup _{z \in \Gamma}\left|e^{-\log (r) z}\right|,
\end{aligned}
$$

where $\operatorname{Len}(\Gamma)=\int_{\Gamma}|d z|$ is the length of $\Gamma$.

Observe now that $\left|e^{-\log (r) z}\right|=r^{-\Re(z)}$ and recall that $\epsilon \leq \Re(z) \leq k+1-\epsilon$, for all $z \in \Gamma$. This implies that

$$
\left|e^{-\log (r) z}\right| \leq r^{-(k+1)+\epsilon} 1_{(0,1)}(r)+r^{-\epsilon} 1_{[1, \infty)}(r),
$$

which in view of (5.20) yields (5.19).

Now, by Corollary 5.6, the measure $\chi$ in (5.11) is the spectral measure of a covariance $\mathcal{H}$-self-similar $\mathrm{IRF}_{k}$, provided (5.12) holds. This, however, readily follows from (5.19). Indeed, by (A.19), the integral in (5.12) is bounded above by

$$
\|\sigma(\mathbb{S})\|_{\mathrm{tr}} \int_{0}^{1} r^{2 k+2}\left\|r^{-\mathcal{H}}\right\|_{\mathrm{op}}^{2} r^{-1} d r+\|\sigma(\mathbb{S})\|_{\mathrm{tr}} \int_{1}^{\infty}\left\|r^{-\mathcal{H}}\right\|_{\mathrm{op}}^{2} r^{-1} d r,
$$

where we used the fact that $\left\|r^{-\mathcal{H}}\right\|_{\mathrm{op}}=\left\|r^{-\mathcal{H}^{*}}\right\|_{\mathrm{op}}$. By (5.19), the integrals in (5.21) are finite and the proof is complete.

Remark 5.9. The sufficient condition in (5.18) may appear restrictive. In particular, it implies that $\mathcal{H}$ has a bounded inverse $(\operatorname{since} 0 \notin \mathrm{sp}(\mathcal{H})$ ). This condition is not all that restrictive when the Hilbert space $\mathbb{V}$ is finite-dimensional and our sufficient conditions are precisely the same as the existing literature in the special case $k=0$ (see e.g., Didier and Pipiras, 2011; Didier et al., 2018).

\subsection{Related work and examples}

Here, we first specialize the results from the previous section and discuss existing related work when $\mathbb{V}$ is finite-dimensional. Then, we consider a class of stationary infinite-dimensional processes, which admit higher-order tangent fields under operator scaling.

Example 5.10 (IRF $F_{0}$ or operator fractional Brownian motions). When $k=0, d=1$, and $\mathbb{V}=\mathbb{R}^{m}$, the $\mathrm{IRF}_{k}$ processes can be identified with the well-studied class of vectorvalued stationary increment processes. The seminal paper of the Didier and Pipiras (2011) established the spectral representation and stochastic integral representations for essentially all Gaussian operator self-similar processes with stationary increments taking values in $\mathbb{R}^{m}$. We demonstrate next how these processes, known as operator fractional Brownian motions (OFBM), can be recovered from our Theorem 5.3. In this setting the operator $\mathcal{H}$ is a real $m \times m$ matrix with eigenvalues $\lambda_{i} \in \mathbb{C}, i=1, \cdots, m$ such that

$$
0<\Re\left(\lambda_{i}\right)<1,
$$


(see e.g., (1.4) in Didier and Pipiras, 2011). Observe that the last condition coincides with (5.18) of Proposition 5.8 for $k=0$.

Let $\{Y(\lambda)\}$ be a zero-mean Gaussian $\mathcal{H}$-self-similar $\mathrm{IRF}_{0}$. Then, if one considers

$$
\lambda_{t}(d u):=\delta_{t}(d u)-\delta_{0}(d u), \quad t \in \mathbb{R},
$$

the process $B(t):=Y\left(\lambda_{t}\right), t \in \mathbb{R}$ has stationary increments. The $\mathcal{H}$-self-similarity of $\{B(t)\}$ follows readily from the self-similarity of $\{Y(\lambda)\}$ and the fact that $c \cdot \lambda_{t}=\lambda_{c t}, c>$ $0, t \in \mathbb{R}$. Conversely, every $\mathcal{H}$-self-similar stationary increment process $\{B(t), t \in \mathbb{R}\}$ can be taken as a representer of an $\mathcal{H}$-self-similar $\mathrm{IRF}_{0}$ process.

Since $\widehat{\lambda_{t}}(x)=e^{\mathrm{it} x}-1$ and $\mathbb{S}=\{-1,1\}$, Relation (5.9) yields

$$
\begin{aligned}
B(t) \equiv Y\left(\lambda_{t}\right) & =\int_{0}^{\infty} \int_{\{-1,1\}}\left(e^{\mathrm{i} t r \theta}-1\right) W(d r, d \theta) \\
& =\int_{0}^{\infty}\left(e^{\mathrm{i} x t}-1\right) W(d x,\{1\})+\int_{0}^{\infty}\left(e^{-\mathrm{i} x t}-1\right) W(d x,\{-1\}) .
\end{aligned}
$$

Now, following the notation in Theorem 3.1 of Didier and Pipiras (2011), let $\widetilde{B}(d x)=$ $\widetilde{B}_{1}(d x)+\mathrm{i} \widetilde{B}_{2}(d x)$, where $\widetilde{B}_{i}, i=1,2$ are independent zero-mean Gaussian $\mathbb{R}^{m}$-valued measures such that $\widetilde{B}_{1}(d x)=\widetilde{B}_{1}(-d x), \widetilde{B}_{2}(d x)=-\widetilde{B}_{2}(-d x)$, and

$$
\mathbb{E}\left[\widetilde{B}(d x) \widetilde{B}(d x)^{*}\right] \equiv \mathbb{E}\left[\widetilde{B}(d x){\left.\overline{\widetilde{B}}(d x)^{\top}\right]}^{\top}=\mathrm{I}_{m} d x\right.
$$

Observe that, by (5.10),

$$
\{W(d x,\{ \pm 1\}), d x \in(0, \infty)\} \stackrel{d}{=}\left\{x^{-(\mathcal{H}+1 / 2)} A_{ \pm 1} \widetilde{B}( \pm d x), d x \in(0, \infty)\right\},
$$

where $A_{ \pm 1} A_{ \pm 1}^{*}=\sigma(\{ \pm 1\})$. Therefore, Relation (5.23) yields

$$
\{B(t)\} \stackrel{d}{=}\left\{\int_{-\infty}^{\infty} \frac{\left(e^{\mathrm{i} x t}-1\right)}{\dot{\mathrm{i}} x}\left(x_{+}^{-(\mathcal{H}-1 / 2)} A_{1}+x_{-}^{-(\mathcal{H}-1 / 2)} A_{-1}\right) \widetilde{B}(d x)\right\} .
$$

This is precisely the representation established in Theorem 3.1 of Didier and Pipiras (2011), wherein $A_{-1}=\overline{A_{1}}$ is the complex conjugate of $A_{1}$ since they consider real-valued processes. Indeed, the last stochastic integral is real-valued if and only if the integrand $f_{t}(x)$ is a Hermitian function of $x$, i.e., $f_{t}(-x)=\overline{f_{t}(x)}$. This is the case, if and only if $A_{1}=\bar{A}_{-1}$.

Remark 5.11. Note that the condition (5.22) on the eigenvalues of the matrix $\mathcal{H}$ does not imply in general that $T_{c}:=c^{\mathcal{H}}, c>0$ are scaling actions in the Euclidean norm of $\mathbb{V} \equiv \mathbb{R}^{m}$. This is because the monotonicity of the function $c \mapsto\left\|c^{\mathcal{H}}\right\|$ may be violated except when the matrix $\mathcal{H}$ is normal (i.e., diagonalizable in an orthonormal basis). In particular, Lemma A.1 is not applicable. Nevertheless, as shown in (Lemma 6.1.5 in Meerschaert and Scheffler, 2001), there is a suitable norm in $\mathbb{V}$, with respect to which the latter are monotone increasing and in this new (equivalent norm) $\left\{c^{\mathcal{H}}, c>0\right\}$ is a scaling action in the sense of Definition 2.7. See also Jurek (1984) for the case where $\mathbb{V}$ is a Banach space.

Remark 5.12. By Proposition 5.8 (with $k=0$ ), Condition (5.22) implies that the stochastic integrals in (5.23) are well-defined.

Example 5.13 (Operator fractional Brownian fields). Stationary increment vector-valued random fields ( $\mathrm{IRF}_{k}$ with $k=0$ ) where $d \geq 2$ have been actively studied by many authors (see e.g., Biermé et al., 2007; Li and Xiao, 2011; Baek et al., 2014; Didier et al., 2018, among others.) In the latter references, self-similarity is considered under operator 
rescaling of both the range and the domain of the process. Here, we consider only scalar rescaling of the domain. In this setting, in the special case of processes taking values in $\mathbb{R}^{m}$ and $k=0$, Theorem 5.3 recovers Proposition 3.1 of Didier et al. (2018).

Interestingly, using Fréchet differentiability in Theorem 5.3, we extend the disintegration formula in Relation (3.10) of Didier et al. (2018) to the case of processes taking values in a separable Hilbert space as well as to the general case of intrinsic random functions of order $k$. We anticipate that a version of our Theorem 5.3 holds under operator scaling of both the range and the domain of $Y$.

Remark 5.14 (The non-uniqueness of the operator exponent $\mathcal{H}$ ). Suppose that $Y$ is an operator $\mathcal{H}$-self-similar zero-mean Gaussian continuous $\mathrm{IRF}_{k}$ taking values in the real Hilbert space $\mathbb{V}_{\mathbb{R}}$ (recall Section 4.3). Then, the distribution of $Y$ is determined by its covariance structure, i.e., by the unique pair of its spectral characteristics $(\chi, \mathcal{Q})$ or equivalently $(\sigma, \mathcal{Q})$. The operator exponent $\mathcal{H}$, however, is not necessarily unique even when $\mathcal{H}$ is restricted to the support of the process $Y$. For the notion of a support of $Y$ and its relation to the operator exponent $\mathcal{H}$, see Section S.6 in Shen et al. (2020). To gain some intuition, suppose that for an operator $\mathcal{A}$ on $\mathbb{V}:=\operatorname{supp}(Y)$, we have that $\left\{c^{\mathcal{A}} Y(\lambda)\right\} \stackrel{d}{=}\{Y(\lambda)\}$, for all $c>0$. If $\mathcal{H}$ and $\mathcal{A}$ commute, then $c^{\mathcal{H}+\mathcal{A}}=c^{\mathcal{H}} c^{\mathcal{A}}, c>0$ and hence $Y$ is also $(\mathcal{H}+\mathcal{A})$-self-similar.

As shown in Didier et al. (2017) such non-uniqueness can arise even in the finitedimensional case with $k=0$, where a wealth of interesting phenomena emerge. Specifically, Theorem 2.4 therein characterizes all possible operator exponents and shows that one can always choose a commuting exponent $\mathcal{H}_{0}$ such that $\mathcal{H}_{0} \mathcal{A}=\mathcal{A H}_{0}$. In their terminology, the operator $\mathcal{A}$ belongs to the tangent space of the group of symmetries of the process. Notice that $\mathcal{A}$ can indeed be viewed as a tangent since it is the Fréchet derivative of $f(c)=c^{\mathcal{A}}$ at $c=1$.

Understanding the non-uniqueness of the operator self-similarity exponent in the general infinite-dimensional case is a challenging problem. We anticipate that the extension of the important characterization results of Didier et al. (2017) to the infinitedimensional case is possible but considerably beyond the scope of this paper.

We end this section with an example of stationary Gaussian $\mathbb{V}$-valued processes, which admit a large class of tangent fields.

Example 5.15 (Higher-order tangent fields in infinite dimensions). In this example, we shall assume that $\mathbb{V}_{\mathbb{R}}$ is a real Hilbert space and through the method of complexification define $\mathbb{V}=\mathbb{V}_{\mathbb{R}}+\dot{i} \mathbb{V}_{\mathbb{R}}$, with the natural inclusion $\mathbb{V}_{\mathbb{R}} \subset \mathbb{V}$.

Consider polar coordinates in $\mathbb{R}^{d} \backslash\{0\}$, where $u=\|x\|, \theta:=x /\|x\|$, are the radial and angular components of $x \in \mathbb{R}^{d} \backslash\{0\}$ and let $\mu(d \theta)$ be a finite, real, $\mathbb{T}_{+}$-valued measure on the unit sphere $\mathbb{S}=\left\{\theta \in \mathbb{R}^{d}:\|\theta\|=1\right\}$. Define the real, $\sigma$-finite $\mathbb{T}_{+}$-valued measure

$$
\nu(d x)=\nu(d u, d \theta)=d u \mu(d \theta), \quad(u, \theta) \in(0, \infty) \times \mathbb{S} .
$$

Let $W_{\mathbb{R}}=\left\{W_{\mathbb{R}}(A), A \in \mathcal{B}\left(\mathbb{R}^{d} \backslash\{0\}\right)\right\}$ and $W_{\mathbb{I}}=\left\{W_{\mathbb{I}}(A), A \in \mathcal{B}\left(\mathbb{R}^{d} \backslash\{0\}\right)\right\}$ be two independent, real (i.e., $\mathbb{V}_{\mathbb{R}}$-valued) orthogonal Gaussian measures with the same control measure $2^{-1} \nu$ (in the sense of Definition 4.6). Construct

$$
W(A)=W_{\mathbb{R}}(A)+\dot{\mathrm{i}} W_{\mathrm{I}}(A) .
$$

It is straightforward to see that $W=\{W(A)\}$ is an orthogonal Gaussian $\mathbb{V}$-valued random measure with control measure $\nu$, i.e., for all bounded Borel $A, B \in \mathcal{B}\left(\mathbb{R}^{d} \backslash\{0\}\right)$, the random vectors $W(A)$ and $W(B)$ are such that

$$
\mathbb{E}[W(A) \otimes W(B)]=\nu(A \cap B)=\int_{0}^{\infty} \int_{\mathbb{S}} 1_{A \cap B}(u \theta) d u \mu(d \theta) .
$$


Note, moreover, that $W$ is also independently scattered, i.e., $W\left(A_{i}\right), i=1, \cdots, n$ are independent for all disjoint bounded Borel sets $A_{i} \subset \mathbb{R}^{d} \backslash\{0\}, i=1, \cdots, n$, which is not necessarily true for all orthogonal Gaussian random measures taking values in a complex Hilbert space.

By analogy with the scalar $\mathbb{C}$-valued case the Gaussian random measure $W$ in (5.25) will be referred to as standard. Since its real and imaginary components are iid, the distribution of the process $W=\{W(A)\}$ is completely determined by its cross-covariance operators in (5.26). Moreover, $W$ has circular symmetry and self-similarity properties:

$$
\left\{e^{\mathrm{i} \varphi} W(d u, d \theta)\right\} \stackrel{f d d}{=}\{W(d u, d \theta)\} \quad \text { and } \quad\left\{r^{1 / 2} W(d u, d \theta)\right\} \stackrel{f d d}{=}\{W(d(r \cdot u), d \theta)\},
$$

for all $\varphi \in \mathbb{R}$ and $r>0$.

Let $\mathcal{H}$ be a bounded linear operator on $\mathbb{V}$ such that

$$
\Re(\operatorname{sp}(\mathcal{H})) \subset(0, k+1),
$$

for some $k \geq 0, k \in \mathbb{Z}$. Suppose also that $\mathcal{A}(\theta), \theta \in \mathbb{S}$ is a collection of bounded linear operators such that $\theta \mapsto \mathcal{A}(\theta)$ is Borel measurable in $\theta$ and such that

$$
\int_{\mathbb{S}}\|\mathcal{A}(\theta)\|_{\text {op }}^{2}\|\mu\|_{\operatorname{tr}}(d \theta)<\infty
$$

where $\|\mu\|_{\text {tr }}$ denoted the (finite) trace measure $\|\mu\|_{\text {tr }}(A):=\|\mu(A)\|_{\text {tr }}$.

Proposition 5.16. Suppose that (5.28) and (5.29) hold for some $k \geq 0, k \in \mathbb{Z}$.

(i) For all $s \in \mathbb{R}^{d}$, the stochastic integral

$$
X(s):=\int_{0}^{\infty} \int_{\mathbb{S}} f_{s}(u, \theta) W(d u, d \theta), \text { where } f_{s}(u, \theta):=e^{\mathrm{i} u s^{\top} \theta}(1 \wedge u)^{k+1} u^{-(\mathcal{H}+1 / 2)} \mathcal{A}(\theta)
$$

exists and defines a stationary $\mathbb{V}$-valued Gaussian random field.

(ii) The process $X=\left\{X(s), s \in \mathbb{R}^{d}\right\}$ has a version with $\gamma$-Hölder continuous paths for all $\gamma \in(0,1 \wedge \epsilon)$ and $\epsilon>0$ such that $\Re(\operatorname{sp}(\mathcal{H})) \subset(\epsilon, k+1)$ (recall (5.28)).

(iii) The continuous-path version of the process $\{X(s)\}$ has a $k$-th order tangent field at each (any) $s_{0}$. More precisely,

$$
\left\{r^{-\mathcal{H}} X\left(s_{0}+r \cdot \lambda\right), \lambda \in \Lambda_{k}\right\} \stackrel{d}{\longrightarrow} Y=\left\{Y(\lambda), \lambda \in \Lambda_{k}\right\}, \quad \text { as } r \downarrow 0,
$$

where the tangent process is an $\mathcal{H}$-self-similar IRF $F_{k}$ given by

$$
Y(\lambda)=\int_{\mathbb{R}^{d}} \widehat{\lambda}(u \theta) u^{-\mathcal{H}-1 / 2} \mathcal{A}(\theta) W(d u, d \theta) .
$$

The proof of this result is given in Section A.6, below.

Remark 5.17. Notice that when $\mathbb{V}$ is infinite-dimensional in (5.30) one cannot consider Gaussian measures $W$ with the control measure equal to the Lebesgue measure times the identity operator $\mathrm{I}_{m}$ as in (5.24). Indeed, for $W(A)$ to be a bona fide random element in $\mathbb{V}$ the control measure of $W$ must take values in $\mathbb{T}_{+}$. This is the key reason why we consider control measures of the type $d u \mu(d \theta)$. In the finite-dimensional case, one can obtain more familiar, but ultimately equivalent stochastic integral representations, in terms of Gaussian $\mathbb{C}^{m}$-valued Gaussian random measures with the Lebesgue control measure times the identity by considering $\mathbb{E}\left[W_{\mathrm{Leb}}(d r, d \theta) \otimes W_{\mathrm{Leb}}(d r, d \theta)\right]=v_{d} r^{d-1} d \theta \times \mathrm{I}_{m}$, where $v_{d}:=\pi^{d / 2} / \Gamma(1+d / 2)$ is the volume of the unit sphere in $\mathbb{R}^{d}$. In this case, the stochastic integral in (5.30) can be equivalently written in Cartesian coordinates as follows:

$$
X(s):=v_{d}^{-1 / 2} \int_{\mathbb{R}^{d}} e^{\mathrm{is} s^{\top} x}(1 \wedge\|x\|)^{k+1}\|x\|^{-(\mathcal{H}+d / 2)} \mathcal{A}(x /\|x\|) W_{\mathrm{Leb}}(d x), \quad s \in \mathbb{R}^{d} .
$$




\subsection{Scalar actions}

In this section, we characterize the spectral measure of covariance self-similar $\operatorname{IRF}_{k}$ 's with respect to the usual scalar scaling action. In this special but important case we obtain a more complete picture of the $H$-self-similar $\operatorname{IRF}_{k}$ 's, where now $H$ is a scalar exponent.

Proposition 5.18. Let $Y$ be a non-constant continuous $I R F_{k}, k \geq 0$ with operator autocovariance function $\mathcal{K}$ and spectral characteristics $(\chi, \mathcal{Q})$. If $Y$ is covariance self-similar with exponent $H$, then $H \in(0, k+1]$ and we have the following dichotomy:

(i) If $H=k+1$, then $\chi \equiv 0$ and if $0<H<k+1$, then $\mathcal{Q}$ is trivial, i.e., $\mathcal{Q}(\nu)=0$, for all $\nu \in \Lambda_{2 k+1}$.

(ii) In the case $0<H<k+1$, the measure $\chi_{k}$ in (5.5) satisfies the scaling property in (5.6) and consequently, the disintegration formula in (5.8) reads:

$$
\chi_{k}(d r d \theta)=r^{-2 H-1} d r \sigma(d \theta), \quad(r, \theta) \in(0, \infty) \times \mathbb{S},
$$

for some finite $\mathbb{T}_{+}$-valued measure $\sigma$ on $\mathbb{S}$.

(iii) Conversely, for every $0<H<k+1$ and any finite $\mathbb{T}_{+}$-valued measure $\sigma$ on $\mathbb{S}$, there exists a covariance $H$-self-similar $I R F_{k}$ with spectral measure $\chi$ such that (5.32) holds, which can be written as in (5.9).

The proof is given in Section A.7, below.

In view of (4.13) and (5.32), one can obtain explicit formulae for the generalized covariance $\mathcal{K}$ of all covariance $H$-self-similar $\mathrm{IRF}_{k}$ 's. This is done next.

Theorem 5.19. Let $\mathcal{K}(\cdot)$ be the generalized covariance of a covariance self-similar $I R F_{k}, k \geq 0$ with exponent $H \in(0, k+1)$. Then, with $\sigma$ as in (5.32), we have:

(i) If $2 H \notin\{1, \ldots, k\}$ is non-integer, then for all $\nu \in \Lambda_{2 k+1}$,

$$
\mathcal{K}(\nu)=I(H) \int_{\mathbb{S}^{d-1}}|(\theta, \cdot)|^{2 H}(\nu) \sigma(d \theta)+\dot{\mathrm{i}} J(H) \int_{\mathbb{S}^{d-1}}(\theta, \cdot)^{<2 H>}(\nu) \sigma(d \theta),
$$

where $(\theta, t)=\theta^{\top} t$ denotes the Euclidean inner product, $x^{<H>}:=\operatorname{sign}(x)|x|^{H}$, and $f(\cdot)(\nu):=\int f(t) \nu(d t)$. Here the real functions $I(H)$ and $J(H)$ are such that

$$
I(H)+\dot{\mathbb{1}} J(H):=\int_{0}^{\infty}\left(e^{\mathrm{i} r}-\sum_{j=0}^{\lfloor 2 H\rfloor} \frac{(\mathrm{i} r)^{j}}{j !}\right) \frac{d r}{r^{2 H+1}} .
$$

(ii) If $2 H \in\{1, \ldots, k\}$ is integer, then for $2 H$ even:

$$
\mathcal{K}(\nu)=\int_{\mathbb{S}^{d-1}}\left[|(\theta, \cdot)|^{2 H}\left(\frac{(-1)^{H+1}}{(2 H) !} \log |(\theta, \cdot)|+\dot{\mathbb{1}} J(H) \operatorname{sign}(\theta, \cdot)\right)\right](\nu) \sigma(d \theta),
$$

and for $2 H$ odd:

$$
\mathcal{K}(\nu)=\int_{\mathbb{S}^{d-1}}\left[|(\theta, \cdot)|^{2 H}\left(I(H)+\dot{\mathbb{i}} \frac{(-1)^{H+1 / 2}}{(2 H) !} \operatorname{sign}(\theta, \cdot) \log |(\theta, \cdot)|\right)\right](\nu) \sigma(d \theta) .
$$

The proof of this result is given in Section A.7, below.

Remark 5.20. Gel'fand and Vilenkin (1964) provide spectral theory for generalized random fields taking values in the dual of the Schwartz space on $\mathbb{R}^{d}$ with homogeneous $(1+k)$ th-order increments (denoted as G-IRF $k$ here). Dobrushin (1979) then studied the self-similar G-IRF $k$ and obtained results similar to Proposition 5.18 where the selfsimilarity parameter $H$ can take any value in $(-\infty, k+1]$. The G-IRF $F_{k}$ class of processes is more general than the $\operatorname{IRF}_{k}$ 's studied by Matheron (1973) and they do not always have a representation on $\mathbb{R}^{d}$. Specifically, it can be shown that a Gaussian self-similar G-IRF $F_{k}$ has a representation as in (4.6) on $\mathbb{R}^{d}$ only if $H>0$ (see Shen, 2019). 
Remark 5.21 (On symmetry and covariance (ir)reversibility). Observe that for $\sigma$ in (5.33), one can write $\sigma=\sigma_{\mathrm{s}}+\sigma_{\mathrm{a}}$, where $\sigma_{\mathrm{s}}(A):=(\sigma(A)+\sigma(-A)) / 2$ and $\sigma_{\mathrm{a}}(A):=$ $(\sigma(A)-\sigma(-A)) / 2$, are the symmetric and anti-symmetric components of $\sigma$. Thus,

$$
\int_{\mathbb{S}^{d-1}}|(\theta, \cdot)|^{2 H}(\nu) \sigma_{\mathrm{a}}(d t)=\int_{\mathbb{S}^{d-1}}(\theta, \cdot)^{<2 H>}(\nu) \sigma_{\mathrm{S}}(d t)=0
$$

and (5.33) can be equivalently written as:

$$
\mathcal{K}(\nu)=I(H) \int_{\mathbb{S}^{d-1}}|(\theta, \cdot)|^{2 H}(\nu) \sigma_{\mathbf{s}}(d \theta)+\dot{\mathrm{i}} J(H) \int_{\mathbb{S}^{d-1}}(\theta, \cdot)^{<2 H>}(\nu) \sigma_{\mathrm{a}}(d \theta) .
$$

This shows that unless $\sigma_{\mathrm{a}} \equiv 0$, we have that $\mathcal{K}(\nu) \neq \mathcal{K}(-\nu)$, for some $\nu \in \Lambda_{2 k+1}$. Recall that by $-\nu$ we understand $((-1) \cdot \nu)(d x):=\nu(-d x)$.

Recall Definition 4.19; $Y$ is covariance reversible, i.e., $\{Y(-\lambda)\}$ and $\{Y(\lambda)\}$ have the same covariance structure, if and only if $\mathcal{K}(\nu)=\mathcal{K}(-\nu), \forall \nu \in \Lambda_{2 k+1}$ or equivalently if and only if $\sigma_{\mathrm{a}} \equiv 0$ (see also Proposition 4.20 above as well as Theorem 5.1 in Didier and Pipiras, 2011, for a related result).

Remark 5.22 (Real $H$-self-similar $\mathbb{I R F}_{k}{ }^{\prime} \mathrm{s}$ ). Recall that $\mathbb{V}=\mathbb{V}_{\mathbb{R}}+\dot{i} \mathbb{V}_{\mathbb{R}}$ (cf Section 4.3). Thus for an $H$-self-similar $\operatorname{IRF}_{k} Y$, we have

$$
Y(\lambda)=\Re Y(\lambda)+\dot{i} \Im Y(\lambda),
$$

where the real and imaginary parts $\Re Y$ and $\Im Y$ are real, i.e., $\mathbb{V}_{\mathbb{R}}$-valued. Thus, in view of (5.35), one can see that $Y$ is real-valued (i.e., $\Im Y \equiv 0$ ) if and only if $\sigma_{s}$ is real and $\sigma_{a}$ imaginary, i.e., if $\sigma$ is Hermitian, $\sigma(A)=\bar{\sigma}(-A)$ for all $A \in \mathcal{B}(\mathbb{S})$. Observe that $Y$ need not be covariance-reversible for it to be real (see Section 4.3.)

Since the covariance structure characterizes completely the zero-mean Gaussian processes taking values in real Hilbert spaces, Theorem 5.19 with Hermitian $\sigma$ provides a complete characterization of all $H$-self-similar $\mathbb{V}_{\mathbb{R}}$-valued $\mathrm{IRF}_{k}$ 's.

Remark 5.23 ( $n$-th order fractional Brownian motion). Perrin et al. (2001) have studied the so-called $n$-th order fractional Brownian motion defined (in Remark 2 therein) as

$$
B_{H}^{(n)}(t):=\frac{1}{2 \pi} \int_{-\infty}^{\infty} \frac{1}{(\dot{\mathrm{i}} \omega)^{H+1 / 2}}\left(e^{\mathrm{i} t \omega}-\sum_{\ell=0}^{n-1} \frac{(\mathrm{i} t \omega)^{\ell}}{\ell !}\right) W(d \omega),
$$

with $n-1<H<n, n \in \mathbb{N}$, where $W(d \omega)$ is a zero mean complex Gaussian measure on $\mathbb{R}$ with the Lebesgue control measure and such that $W(-d \omega)=\overline{W(d \omega)}$. Notice, however, that the above integral representation is well defined only if $H \in(n-1, n)$. While one can always put $n:=\lceil H\rceil$, the integer values of $H$ have to be dealt with separately. Using our abstract approach, we can handle all values of $H \in(0, k+1), k:=n-1$, in a unified manner.

Indeed, observe that with $k=n-1$, for any $\lambda \in \Lambda_{k}$, we have

$$
\begin{aligned}
B_{H}^{(n)}(\lambda) & =\frac{1}{2 \pi} \int_{-\infty}^{\infty} \widehat{\lambda}(\omega)(\dot{\mathrm{i}} \omega)^{-(H+1 / 2)} W(d \omega) \\
& =\frac{1}{2 \pi} \int_{0}^{\infty} \int_{\mathbb{S}} \widehat{\lambda}(u \theta) u^{-(H+1 / 2)} e^{-\mathrm{i} \theta \frac{\pi}{2}(H+1 / 2)} W(d u, d \theta) \\
& =: \frac{1}{2 \pi} \int_{0}^{\infty} \int_{\mathbb{S}} \widehat{\lambda}(u \theta) u^{-(H+1 / 2)} \widetilde{W}(d u, d \theta),
\end{aligned}
$$

where we used the change to polar coordinates $(u, \theta):=(|\omega|, \operatorname{sign}(\omega))$, with $\mathbb{S}=\{ \pm 1\}$ and the fact that $\lambda$ annihilates all polynomials of degree up to $k$. This latter integral is defined for all $0<H<k+1$. Notice that $\widetilde{W}(d u, d \theta):=e^{-\mathrm{i} \theta \frac{\pi}{2}(H+1 / 2)} W(d u, d \theta)$ is equal in law to $W(d u, d \theta)$ and (5.36) is a particular case of the stochastic representation of the self-similar $\mathrm{IRF}_{k}$ 's characterized in Proposition 5.18. 
We end with an example outlining the general form of the $\mathbb{R}$-valued $H$-self-similar Gaussian $\mathrm{IRF}_{k}$ 's in $\mathbb{R}^{d}$, which may be viewed as generalized fractional Brownian fields with $(1+k)$-th order stationary increments.

Example 5.24 (Real $n$-th order fractional Brownian fields). Fix an integer $k:=n-1 \geq 0$, and let $\lambda_{t}$ be as in (2.7). Let also $\sigma(d \theta)$ be a finite symmetric measure on the unit sphere $\mathbb{S} \subset \mathbb{R}^{d}$. For all $H \in(0, k+1)$, it can be shown that $f_{t}(x, \theta):=(\langle\cdot, \theta\rangle-x)_{+}^{H-1 / 2}\left(\lambda_{t}\right)$ belongs to $\mathcal{L}^{2}(d x, \sigma(d \theta))$, where $d x$ is the Lebesgue measure on $\mathbb{R}$. Thus, one can define the $\mathbb{R}$-valued Gaussian random field

$$
Y(t):=\int_{-\infty}^{\infty} \int_{\mathbb{S}}(\langle\cdot, \theta\rangle-x)_{+}^{H-1 / 2}\left(\lambda_{t}\right) W(d x, d \theta), \quad t \in \mathbb{R}^{d},
$$

where $W(d x, d \theta)$ is a zero-mean Gaussian real-valued random measure on $\mathbb{R} \times \mathbb{S}$ with control measure $d x \sigma(d \theta)$. Then, it is easy to see with a simple change of variables that $Y(\lambda):=\int Y d \lambda, \lambda \in \Lambda_{k}$ is an $H$-self-similar $\mathrm{IRF}_{k}$ with real and symmetric generalized covariance $K$. In this case Theorem 5.19 yields:

$$
K(\nu)=C_{H} \int_{\mathbb{S}}\left[|\langle\cdot, \theta\rangle|^{2 H}\left(I(H)+\frac{(-1)^{H+1}}{(2 H) !} 1_{\mathbb{N}}(H) \log |\langle\cdot, \theta\rangle|\right)\right](\nu) \sigma(d \theta) .
$$

For more examples and further insights, see the PhD thesis of Shen (2019).

\section{A Proofs and auxiliary results}

\section{A.1 Proofs and tools for Section 2}

Proof of Lemma 2.2: Notice that $S_{c}\left(\breve{\Lambda}_{k}, \mathbb{V}\right)$ is a closed set in the metric space $\left(C\left(\mathbb{R}^{d}, \mathbb{V}\right), \rho\right)$ of continuous $\mathbb{V}$-valued functions on $\mathbb{R}^{d}$, equipped with the metric $\rho$ in (2.13). Thus, it is enough to show that $\left(C\left(\mathbb{R}^{d}, \mathbb{V}\right), \rho\right)$ is a complete separable metric space (cf. Theorems 1 and 2 in Chapter XIV.2 in Kuratowski, 1977).

It is known that the metric $\rho$ generates the compact-open topology (see, e.g., Theorem 46.8 in Munkres, 2000). Therefore, to prove separability it is enough to demonstrate that this topology has a countable base. Recall that the compact-open topology on $C\left(\mathbb{R}^{d}, \mathbb{V}\right)$ has a sub-base comprising all sets $V(K, U)=\left\{f \in C\left(\mathbb{R}^{d}, \mathbb{V}\right): f(K) \subset U\right\}$, where $K \subset \mathbb{R}^{d}$ is compact and $U \subset \mathbb{V}$ is open. Since $\left(\mathbb{V}, d_{\mathbb{V}}\right)$ is separable and $\mathbb{R}^{d}$ is locally compact, the compact-open topology on $C\left(\mathbb{R}^{d}, \mathbb{V}\right.$ ) is second countable (cf. Theorem 5.2, page 265 in Dugundji, 1966). This entails the separability of $\left(C\left(\mathbb{R}^{d}, \mathbb{V}\right), \rho\right)$.

Completeness is established in a standard manner. Let $\left\{f_{n}\right\}$ be a Cauchy sequence in $\left(C\left(\mathbb{R}^{d}, \mathbb{V}\right), \rho\right)$. In view of (2.13), for each $t \in \mathbb{R}^{d},\left\{f_{n}(t)\right\}$ is a Cauchy sequence in the complete metric space $\left(\mathbb{V}, d_{\mathbb{V}}\right)$. Thus, $f_{n}(t) \rightarrow f(t) \in \mathbb{V}$. It remains to show that $f$ is continuous and $\rho\left(f_{n}, f\right) \rightarrow 0$. Fix an arbitrary compact $K \subset \mathbb{R}^{d}$ and $\epsilon>0$. Since $\left\{f_{n}\right\}$ is Cauchy in $\rho$, there exists an $N_{\epsilon}$ such that

$$
\sup _{\tau \in K} d_{\mathbb{V}}\left(f_{n}(\tau), f_{m}(\tau)\right) \leq \epsilon / 3, \quad \text { for all } m, n \geq N_{\epsilon} .
$$

On the other hand, for every fixed $t \in K$, we have

$$
d_{\mathbb{V}}\left(f_{n}(t), f(t)\right)=\lim _{m \rightarrow \infty} d_{\mathbb{V}}\left(f_{n}(t), f_{m}(t)\right) \leq \epsilon / 3, \quad n \geq N_{\epsilon} .
$$

Since the latter bound is uniform in $t$, we also obtain $\sup _{t \in K} d_{\mathbb{V}}\left(f_{n}(t), f(t)\right) \leq \epsilon / 3, n \geq N_{\epsilon}$. That is, $f_{n}$ converge to $f$ uniformly on all compact $K$. It remains to establish that $f$ is continuous. For all $s, t \in K$, we have by the triangle inequality that

$$
d_{\mathbb{V}}(f(t), f(s)) \leq 2 \sup _{\tau \in K} d_{\mathbb{V}}\left(f_{n}(\tau), f(\tau)\right)+d_{\mathbb{V}}\left(f_{n}(t), f_{n}(s)\right) \leq \epsilon,
$$


provided that $\|t-s\|<\delta$ for some sufficiently small $\delta>0$. Here, we used the uniform continuity of $f_{n}$ on $K$. Since $\epsilon>0$ was arbitrary, this completes the proof of the (uniform) continuity of $f$ on $K$.

Proof of Lemma 2.10: Properties (i) and (ii) in Definition 2.7 are immediate. We now verify (iii). Consider the coordinate-wise action on $\breve{S}_{c}\left(\Lambda_{k}, \mathbb{V}\right)$, also denoted as $\left\{\widetilde{T}_{a}, a \in \mathbb{R}_{+}\right\}$for convenience. One can easily verify that $\mathcal{J}\left(\widetilde{T}_{a}(f)\right)=\widetilde{T}_{a}(\mathcal{J}(f))$. Let $f_{n} \rightarrow f$ in $S_{c}\left(\Lambda_{k}, \mathbb{V}\right)$ and $a_{n} \rightarrow a>0$. To show that $\rho\left(\widetilde{T}_{a_{n}}\left(f_{n}\right), \widetilde{T}_{a}(f)\right) \rightarrow 0$, it is enough to verify that for every $K>0$, we have

$$
\sup _{\|t\| \leq K} d_{\mathbb{V}}\left(\widetilde{T}_{a_{n}}\left(\breve{f}_{n}\right)(t), \widetilde{T}_{a}(\breve{f})(t)\right) \rightarrow 0, \quad \text { as } n \rightarrow \infty,
$$

where $\breve{f}_{n}=\mathcal{J}\left(f_{n}\right)$ and $\breve{f}=\mathcal{J}(f)$.

In view of Lemma A.2, below, it is enough to show that $\widetilde{T}_{a_{n}}\left(\breve{f}_{n}\right)\left(t_{n}\right) \rightarrow \widetilde{T}_{a}(\breve{f})(t)$, whenever $t_{n} \rightarrow t$ in $\bar{B}_{K}:=\left\{t:\|t\| \leq K, t \in \mathbb{R}^{d}\right\}$. Notice, however, that $\widetilde{T}_{a_{n}}\left(\breve{f}_{n}\right)\left(t_{n}\right)=$ $T_{a_{n}}\left(y_{n}\right)$ and $\widetilde{T}_{a}(\breve{f})(t)=T_{a}(y)$, where $y_{n}:=\breve{f}_{n}\left(t_{n}\right)$ and $y:=\breve{f}(t)$. By applying Lemma A.2 again, but now to the locally converging functions $\breve{f}_{n}$ and $\breve{f}$, we have that $y_{n}=\breve{f}_{n}\left(t_{n}\right) \rightarrow$ $y=\breve{f}(t)$ in $\mathbb{V}$, whenever $t_{n} \rightarrow t$ in $\bar{B}_{K}$. Hence, the continuity of the scaling action $\left\{T_{a}\right\}$, yields $T_{a_{n}}\left(y_{n}\right) \rightarrow T_{a}(y)$ in $\mathbb{V}$, which completes the proof of property (iii).

Let now $0 \neq f \in S_{c}\left(\Lambda_{k}, \mathbb{V}\right)$. Proving property (iv) of Definition 2.7 amounts to showing that $\rho\left(\widetilde{T}_{a_{1}}(f), 0\right)<\rho\left(\widetilde{T}_{a_{2}}(f), 0\right)$, for all $0<a_{1}<a_{2}$. Observe that by property (iv) for $\left\{T_{a}\right\}$, we have

$$
d_{\mathbb{V}}\left(\widetilde{T}_{a_{1}}(\breve{f})(t), 0\right) \equiv d_{\mathbb{V}}\left(T_{a_{1}}(\breve{f}(t)), 0\right) \leq d_{\mathbb{V}}\left(T_{a_{2}}(\breve{f}(t)), 0\right) \equiv d_{\mathbb{V}}\left(\widetilde{T}_{a_{2}}(\breve{f})(t), 0\right) .
$$

This implies that $\rho\left(\widetilde{T}_{a_{1}}(f), 0\right) \leq \rho\left(\widetilde{T}_{a_{2}}(f), 0\right)$. We next argue that the inequality is strict. Since $f \neq 0$, we have $0 \neq \breve{f}(t) \in \mathbb{V}$ for some $t \in \mathbb{R}^{d}$. Let $t \in \bar{B}_{j}$ for some large enough $j$, where $B_{j}$ is as defined in (2.15). Since the suprema therein are attained, it is enough to show that

$$
\begin{aligned}
d_{\mathbb{V}}\left(T_{a_{1}}\left(\breve{f}\left(t_{1}\right)\right), 0\right) & :=\max _{t \in \overline{B_{j}}} d_{\mathbb{V}}\left(T_{a_{1}}(\breve{f}(t)), 0\right) \\
& <\max _{t \in \bar{B}_{j}} d_{\mathbb{V}}\left(T_{a_{2}}(\breve{f}(t)), 0\right)=: d_{\mathbb{V}}\left(T_{a_{2}}\left(\breve{f}\left(t_{2}\right)\right), 0\right) .
\end{aligned}
$$

Observe that, $0<d_{\mathbb{V}}\left(T_{a_{1}}(\breve{f}(t), 0) \leq d_{\mathbb{V}}\left(T_{a_{1}}\left(\breve{f}\left(t_{1}\right)\right), 0\right)\right.$ and hence $\breve{f}\left(t_{1}\right) \neq 0$. Thus, by the radial monotonicity of the action $\left\{T_{a}\right\}$, we have

$$
d_{\mathbb{V}}\left(T_{a_{1}}\left(\breve{f}\left(t_{1}\right)\right), 0\right)<d_{\mathbb{V}}\left(T_{a_{2}}\left(\breve{f}\left(t_{1}\right)\right), 0\right) \leq d_{\mathbb{V}}\left(T_{a_{2}}\left(\breve{f}\left(t_{2}\right)\right), 0\right),
$$

which yields (A.1) and completes the proof of (iv).

We now verify property (v). In view of Remark 2.8 , it is equivalent to show that for all $f \in S_{c}\left(\Lambda_{k}, \mathbb{V}\right)$, we have $\widetilde{T}_{1 / n}(f) \rightarrow 0$ in $S_{c}\left(\Lambda_{k}, \mathbb{V}\right)$. Suppose that this is not the case. Then, for some compact $K \subset \mathbb{R}^{d}$, some $\epsilon_{0}>0$ and a sequence $t_{n} \in K$, we have

$$
d_{\mathbb{V}}\left(T_{1 / n}\left(\breve{f}\left(t_{n}\right)\right), 0\right) \geq \epsilon_{0}>0 .
$$

Since $K$ is compact, for some $n^{\prime} \rightarrow \infty$, we have $t_{n^{\prime}} \rightarrow$ some $t_{*} \in K$, and by the continuity of $\breve{f}$, we have $\breve{f}\left(t_{n}\right) \rightarrow \breve{f}\left(t_{*}\right)$ in $\mathbb{V}$. For all $\delta>0$, fixed, the radial monotonicity implies that

$$
0<\epsilon_{0} \leq \limsup _{n^{\prime} \rightarrow \infty} d_{\mathbb{V}}\left(T_{1 / n^{\prime}}\left(\breve{f}\left(t_{n^{\prime}}\right)\right), 0\right) \leq \lim _{n^{\prime} \rightarrow \infty} d_{\mathbb{V}}\left(T_{\delta}\left(\breve{f}\left(t_{n^{\prime}}\right)\right), 0\right)=d_{\mathbb{V}}\left(T_{\delta}\left(\breve{f}\left(t_{*}\right)\right), 0\right) .
$$

Property (v), for the scaling action $\left\{T_{a}\right\}$, however, entails that $T_{\delta}\left(\breve{f}\left(t_{*}\right)\right) \rightarrow 0$ in $\mathbb{V}$, as $\delta \downarrow 0$, which yields a contradiction with the above inequality and completes the proof.

The following result shows that the linear operator actions considered in Remark 2.9 are in fact actions under mild natural conditions on the operator exponent $\mathcal{H}$. 
Lemma A.1. Let $\mathbb{V}$ be a Hilbert space and $\mathcal{H}: \mathbb{V} \rightarrow \mathbb{V}$ a bounded linear operator such that

$$
\Re(\operatorname{sp}(\mathcal{H})) \subset(0, \infty) \quad \text { and } \quad 2 \Re\langle\mathcal{H} x, x\rangle_{\mathbb{V}}=\left\langle\left(\mathcal{H}+\mathcal{H}^{*}\right) x, x\right\rangle_{\mathbb{V}}>0,
$$

for all $x \neq 0$. Then, $T_{c}:=c^{\mathcal{H}}, c>0$ is a scaling action in the sense of Definition 2.7. Here $\Re(\operatorname{sp}(\mathcal{H})$ ) denotes the set of real parts of the elements in the spectrum of $\mathcal{H}$.

Proof. Properties (i)-(iii) of Definition 2.7 are immediate. Indeed, we have $\left(c_{1} c_{2}\right)^{\mathcal{H}}=$ $c_{1}^{\mathcal{H}} c_{2}^{\mathcal{H}}, c_{1}, c_{2}>0$ and $1^{\mathcal{H}}=\mathrm{I}$, while Property (iii) follows from the strong continuity (in operator norm) of $c \mapsto c^{\mathcal{H}}$. To establish the radial monotonicity (Property (iv) in Definition 2.7), it is enough to show that for all $x \neq 0$, the function $\varphi(c):=\left\langle c^{\mathcal{H}} x, c^{\mathcal{H}} x\right\rangle_{\mathrm{V}}$ is strictly increasing in $c>0$. To this end, we will show that $\varphi^{\prime}(c)>0$ for all $c>0$ and $x \neq 0$. By (5.2), $h^{-1}\left((c+h)^{\mathcal{H}}-c^{\mathcal{H}}\right) \rightarrow \mathcal{H} c^{\mathcal{H}-1}$, in operator norm, as $h \rightarrow 0$. Thus, for $c>0$,

$$
\begin{aligned}
& \varphi^{\prime}(c)= \lim _{h \rightarrow 0} \frac{1}{h}\left(\left\langle(c+h)^{\mathcal{H}} x,(c+h)^{\mathcal{H}} x\right\rangle_{\mathrm{V}}-\left\langle c^{\mathcal{H}} x,(c+h)^{\mathcal{H}} x\right\rangle_{\mathbb{V}}\right. \\
&\left.+\left\langle c^{\mathcal{H}} x,(c+h)^{\mathcal{H}} x\right\rangle_{\mathbb{V}}-\left\langle c^{\mathcal{H}} x, c^{\mathcal{H}} x\right\rangle_{\mathbb{V}}\right) \\
&=\left\langle\mathcal{H} c^{\mathcal{H}-1} x, c^{H} x\right\rangle_{\mathbb{V}}+\left\langle c^{\mathcal{H}}, \mathcal{H} c^{\mathcal{H}-1} x\right\rangle_{\mathbb{V}}=2 c^{-1} \Re\left\langle\mathcal{H} c^{\mathcal{H}} x, c^{\mathcal{H}} x\right\rangle_{\mathbb{V}},
\end{aligned}
$$

which is strictly positive, by assumption. Finally, property (v) follows from the first condition in (A.2) as in the proof of Relation (5.19).

\section{A.2 Proof of Proposition 3.9}

Proposition 3.9 is the key to establishing the a.e. intrinsic stationarity of the tangent fields in Theorem 3.8. This section outlines its proof, which is based on the following lemma and the Egorov and Lusin Theorems.

Lemma A.2. Let $\left(K, \rho_{K}\right)$ be a compact metric space and $\left(E, \rho_{E}\right)$ be a metric space. Suppose that $f_{n}$ and $f: K \rightarrow E$ are Borel measurable functions.

If the function $f$ is continuous, then

$$
\sup _{x \in K} \rho_{E}\left(f_{n}(x), f(x)\right) \rightarrow 0, \quad \text { as } n \rightarrow \infty,
$$

if and only if

$$
\rho_{E}\left(f_{n}\left(x_{n}\right), f(x)\right) \rightarrow 0, \quad \text { whenever } \rho_{K}\left(x_{n}, x\right) \rightarrow 0 .
$$

Proof. ('if') Suppose that (A.4) holds and assume that (A.3) fails. Then, for some $\epsilon_{0}>0$, there exist an infinite sequence $n_{k} \in \mathbb{N}$ and $x_{n_{k}} \in K$, such that $\rho_{E}\left(f_{n_{k}}\left(x_{n_{k}}\right), f\left(x_{n_{k}}\right)\right) \geq$ $\epsilon_{0}>0$. It is easy to see that $\left\{x_{n_{k}}\right\}$ is also an infinite sequence, since for every $k_{0} \in \mathbb{N}$, by (A.4), we have $f_{n_{k}}\left(x_{n_{k_{0}}}\right) \rightarrow f\left(x_{n_{k_{0}}}\right)$, as $n_{k} \rightarrow \infty$. Indeed, had $\left\{x_{n_{k}}\right\}$ been a finite set, for some infinite subsequence $\left\{n_{k}^{\prime}\right\} \subset\left\{n_{k}\right\}$, we would have $x_{n_{k}^{\prime}}=x_{n_{k_{0}}}$ and hence $\rho_{E}\left(f_{n_{k}^{\prime}}\left(x_{n_{k}^{\prime}}\right), f\left(x_{n_{k}^{\prime}}\right)\right)=\rho_{E}\left(f_{n_{k}^{\prime}}\left(x_{n_{k_{0}}}\right), f\left(x_{n_{k_{0}}}\right)\right) \rightarrow 0$, contradicting the construction of the $x_{n_{k}}$ 's.

The infinite sequence $\left\{x_{n_{k}}\right\}$ is included in the compact $K$, and hence it has a converging subsequence $x_{n_{k}(m)} \rightarrow x$. This, in view of (A.4), implies that $f_{n_{k}(m)}\left(x_{n_{k}(m)}\right) \rightarrow f(x)$ in $\mathbb{V}$. Since $f$ is continuous at $x$, however, $f\left(x_{n_{k}(m)}\right) \rightarrow f(x)$. This, by the triangle inequality, implies

$$
\begin{aligned}
& \rho_{E}\left(f_{n_{k}(m)}\left(x_{n_{k}(m)}\right), f\left(x_{n_{k}(m)}\right)\right) \\
& \leq \rho_{E}\left(f_{n_{k}(m)}\left(x_{n_{k}(m)}\right), f(x)\right)+\rho_{E}\left(f(x), f\left(x_{n_{k}(m)}\right)\right) \rightarrow 0, \text { as } n_{k}(m) \rightarrow \infty .
\end{aligned}
$$

This contradicts the assumption that $\rho_{E}\left(f_{n_{k}(m)}\left(x_{n_{k}(m)}\right), f\left(x_{n_{k}(m)}\right)\right) \geq \epsilon_{0}$. 
Tangent fields, intrinsic stationarity, and self similarity

('only if') Let $x_{n} \rightarrow x$. By the triangle inequality, we have that

$$
\begin{aligned}
\rho_{E}\left(f_{n}\left(x_{n}\right), f(x)\right) & \leq \rho_{E}\left(f_{n}\left(x_{n}\right), f\left(x_{n}\right)\right)+\rho_{E}\left(f\left(x_{n}\right), f(x)\right) \\
& \leq \sup _{x^{\prime} \in K} \rho_{E}\left(f_{n}\left(x^{\prime}\right), f\left(x^{\prime}\right)\right)+\rho_{E}\left(f\left(x_{n}\right), f(x)\right),
\end{aligned}
$$

which converges to zero by (A.3) and the continuity of $f$.

The next result is a restatement of Theorem 7.5.1 in Dudley (2002).

Theorem A.3 (Egorov). Let $(B, \mathcal{B}, \mu)$ be a finite measure space and $\left(Y, \rho_{Y}\right)$ be a separable metric space. Suppose that $f_{n}: B \rightarrow Y, n=1, \cdots$ are measurable functions such that, for $\mu$-almost all $x \in B$,

$$
f_{n}(x) \rightarrow f(x) \text {, as } n \rightarrow \infty .
$$

Then, for all $\epsilon>0$, there exists a measurable set $B_{\epsilon} \subset B$, such that

$$
\mu\left(B \backslash B_{\epsilon}\right)<\epsilon \text { and } \sup _{x \in B_{\epsilon}} \rho_{Y}\left(f_{n}(x), f(x)\right) \rightarrow 0 \text {, as } n \rightarrow \infty .
$$

We present next a relatively general form of the classic Lusin's theorem stating that every Borel function is nearly continuous. The proof follows the elegant 3-line argument given in Theorem 1 on page 56 in Loeb and Talvila (2004). We provide a bit more detail and tailor the result to the case of metric spaces.

Theorem A.4 (Lusin). Let $\left(X, \rho_{X}\right)$ be a metric space and $\left(Y, \rho_{Y}\right)$ be a separable metric space. Let also $f: X \rightarrow Y$ be a Borel measurable function and $\mu$ be a finite Borel measure $\mu$ on $X$.

For every $\epsilon>0$, there exists a closed set $F \subset X$, such that $\mu(X \backslash F)<\epsilon$ and $f: F \rightarrow Y$ is continuous. If $\left(X, \rho_{X}\right)$ is separable and complete, then the set $F$ can be taken to be compact.

Proof. We will essentially unpack the argument on page 56 of Loeb and Talvila (2004) with small modifications.

By Theorem 7.1.3 on page 175 in Dudley (2002) every finite Borel measure $\mu$ on $\left(X, \rho_{X}\right)$ is closed regular, that is, for every Borel set $A$ in $X$, we have

$$
\mu(A)=\sup \{\mu(F): F \subset A, F \text { is closed }\} .
$$

Recall that $\mu$ is called regular if the sets $F$ above can be taken to be compact. Ulam's Theorem implies that if $\left(X, \rho_{X}\right)$ is separable and complete, then $\mu$ is regular (cf. Theorem 7.1.4 in Dudley, 2002).

We now fix an $\epsilon>0$ and construct the closed set $F$. Since $\left(Y, \rho_{Y}\right)$ is separable, it is second countable, i.e., its topology has a countable base. Namely, there exists a countable collection of open sets $\left\{V_{n}, n \in \mathbb{N}\right\}$ in $Y$ such that every open set $V \subset Y$ can be represented as a union of $V_{n}$ 's, i.e., $V=\cup\left\{V_{n}: V_{n} \subset V, n \in \mathbb{N}\right\}$.

Following Loeb and Talvila (2004), by (A.5) since $\mu$ is finite, we can find closed sets $F_{n} \subset f^{-1}\left(V_{n}\right)$ and $F_{n}^{\prime} \subset X \backslash f^{-1}\left(V_{n}\right)$ in $X$ (compact if $\mu$ is regular), such that

$$
\mu\left(f^{-1}\left(V_{n}\right) \backslash F_{n}\right)<\frac{\epsilon}{2^{n+1}} \quad \text { and } \quad \mu\left(\left[X \backslash f^{-1}\left(V_{n}\right)\right] \backslash F_{n}^{\prime}\right)<\frac{\epsilon}{2^{n+1}} .
$$

Observe that

$$
\mu\left(X \backslash\left(F_{n} \cup F_{n}^{\prime}\right)\right)=\mu\left(f^{-1}\left(V_{n}\right) \backslash F_{n}\right)+\mu\left(\left[X \backslash f^{-1}\left(V_{n}\right)\right] \backslash F_{n}^{\prime}\right)<\frac{\epsilon}{2^{n}} .
$$


Define $F:=\cap_{n \in \mathbb{N}}\left(F_{n} \cup F_{n}^{\prime}\right)$ and notice that $F$ is closed and in fact compact if $\left(X, \rho_{X}\right)$ is separable and complete. The above relation implies moreover that

$$
\mu(X \backslash F) \leq \sum_{n \in \mathbb{N}} \mu\left(X \backslash\left(F_{n} \cup F_{n}^{\prime}\right)\right)<\sum_{n \in \mathbb{N}} \frac{\epsilon}{2^{n}}=\epsilon .
$$

To complete the proof, it remains to show that $f: F \rightarrow Y$ is continuous. To this end, it is enough to show that for every $x \in F$ and every $V_{n}$ such that $f(x) \in V_{n}$, there is an open set $U \ni x$ such that $f(U \cap F) \subset f\left(V_{n}\right)$. Suppose $f(x) \in V_{n}$ and consider the open set $U:=X \backslash F_{n}^{\prime}$. Since $F \subset F_{n} \cap F_{n}^{\prime}$ and $U \cap F_{n}^{\prime}=\emptyset$, we have

$$
U \cap F \subset U \cap F_{n} \subset f^{-1}\left(V_{n}\right),
$$

which implies $f(U \cap F) \subset V_{n}$. We have thus established the desired continuity of $f$ on F.

Remark A.5. Loeb and Talvila's proof of Lusin's Theorem A.4 is not constructive and it does not use approximation arguments based on the Tietze-Uryson Lemma and Egorov's theorem as many other proofs in the literature (see, e.g., Theorem 7.5.2 in Dudley, 2002). This makes it possible to extend Lusin's theorem to functions taking values in an arbitrary separable metric space.

We conclude this section with the proof of Proposition 3.9.

Proof of Proposition 3.9: By Egorov's Theorem (see Theorem A.3, above), there is a Borel set $B_{\epsilon} \subset B$ such that $\mu\left(B \backslash B_{\epsilon}\right)<\epsilon / 2$ and

$$
\sup _{s \in B_{\epsilon}} \rho_{E}\left(F_{n}(s), G(s)\right) \rightarrow 0,
$$

as $n \rightarrow \infty$. Observe that since the Lebesgue measure is closed regular (recall (A.5)), one can choose the set $B_{\epsilon}$ to be closed. Therefore, $B_{\epsilon}$ with the usual metric in $\mathbb{R}^{d}$ is a complete and separable metric space. Hence, we can apply Lusin's Theorem A.4 to $X:=B_{\epsilon} \subset \mathbb{R}^{d}$ and $Y:=E$ to conclude that there is a further compact set $K_{\epsilon} \subset B_{\epsilon}$, such that $\mu\left(B_{\epsilon} \backslash K_{\epsilon}\right)<\epsilon / 2$ and the function $G: K_{\epsilon} \rightarrow E$ is continuous.

Observe that

$$
\mu\left(B \backslash K_{\epsilon}\right)=\mu\left(B \backslash B_{\epsilon}\right)+\mu\left(B_{\epsilon} \backslash K_{\epsilon}\right)<\epsilon .
$$

By Lemma A.2, the continuity of $G$ on $K_{\epsilon}$ and the uniform convergence (A.6) imply (3.17).

\section{A.3 Supplementary results and proofs for Section 3}

The following convergence to types lemma is rather useful.

Lemma A.6. Let $\left\{T_{a}, a>0\right\}$ be a scaling action on some metric space $\left(\mathbb{X}, d_{\mathbb{X}}\right)$. Let also $\xi, \widetilde{\xi}$ and $\xi_{n}$ be random elements taking values in $\mathbb{X}$. Then the following hold.

(i) If $\xi$ is non-zero, then $T_{a^{\prime}}(\xi) \stackrel{d}{=} T_{a^{\prime \prime}}(\xi)$ implies $a^{\prime}=a^{\prime \prime}$.

(ii) Suppose that $\xi_{n} \stackrel{d}{\rightarrow} \xi$ and $\widetilde{\xi}_{n}:=T_{a_{n}}\left(\xi_{n}\right) \stackrel{d}{\rightarrow} \widetilde{\xi}$, for some sequence $a_{n}>0$, where both $\xi$ and $\widetilde{\xi}$ are non-zero. Then $a_{n} \rightarrow a$ for some $a>0$ and $\widetilde{\xi} \stackrel{d}{=} T_{a}(\xi)$.

(iii) If $\xi$ is non-zero and $T_{a_{n}}(\xi) \stackrel{d}{\rightarrow} 0$, then $a_{n} \rightarrow 0$. 
Proof. Part (i). Suppose that $a^{\prime}<a^{\prime \prime}$. Then,

$$
\xi \stackrel{d}{=} T_{a^{\prime \prime}}^{-1} \circ T_{a^{\prime}}(\xi) \equiv T_{a^{\prime} / a^{\prime \prime}}(\xi),
$$

which implies $\xi \stackrel{d}{=} T_{c_{n}}(\xi)$, for all $n \in \mathbb{N}$, where $c_{n}=\left(a^{\prime} / a^{\prime \prime}\right)^{n} \downarrow 0$. As in Remark 2.8, let $B_{r}$ be the open ball centered at the origin with radius $r$. It follows that, for every $B_{r}$,

$$
\mathbb{P}\left(\xi \in B_{r}\right)=\mathbb{P}\left(T_{c_{n}}(\xi) \in B_{r}\right)=\mathbb{P}\left(\xi \in T_{1 / c_{n}}\left(B_{r}\right)\right) .
$$

Since $1 / c_{n} \rightarrow \infty$, applying (2.15) and (2.17), we see that $\mathbb{P}\left(\xi \in B_{r}\right)=1$, for all $r>0$, which contradicts the assumption that $\xi$ is non-zero.

Part (ii). We will first show that $\left\{a_{n}\right\}$ is bounded away from 0 and $\infty$. Indeed, suppose that $a_{n^{\prime}} \rightarrow \infty$ for some $n^{\prime} \rightarrow \infty$. Observe that all but countably many $B_{r}$ 's are continuity sets for the distribution of $\xi$. Indeed, the sets $\partial B_{r}:=\bar{B}_{r} \backslash B_{r}, r>0$ are pairwise disjoint in $r$ and for each $\epsilon>0$, there are at most $1 / \epsilon$ distinct values for $r$, such that $\mathbb{P}\left(\xi \in \partial B_{r}\right)>\epsilon$.

For every $r>0$ such that $\mathbb{P}\left(\xi \in \partial B_{r}\right)=0$, since $\xi_{n^{\prime}} \stackrel{d}{\rightarrow} \xi$, we have

$$
\begin{aligned}
\mathbb{P}\left(\xi \in B_{r}\right) & =\lim _{n^{\prime} \rightarrow \infty} \mathbb{P}\left(\xi_{n^{\prime}} \in B_{r}\right)=\lim _{n^{\prime} \rightarrow \infty} \mathbb{P}\left(T_{a_{n^{\prime}}}\left(\xi_{n^{\prime}}\right) \in T_{a_{n^{\prime}}}\left(B_{r}\right)\right) \\
& \geq \limsup _{n^{\prime} \rightarrow \infty} \mathbb{P}\left(\widetilde{\xi}_{n^{\prime}} \in T_{m}\left(B_{r}\right)\right),
\end{aligned}
$$

where $\widetilde{\xi}_{n^{\prime}}:=T_{a_{n^{\prime}}}\left(\xi_{n^{\prime}}\right)$ and $m$ is an arbitrary fixed integer. Here, we used the fact that $T_{m}\left(B_{r}\right) \subset T_{a_{n^{\prime}}}\left(B_{r}\right)$, for all large enough $n^{\prime}$, by (2.15) and (2.17).

Now, since $T_{m}$ is a homeomorphism, we have $\partial T_{m}\left(B_{r}\right)=T_{m}\left(\partial B_{r}\right)$ are disjoint in $r>0$, and by the above argument, for all but countably many $r^{\prime}$ s, we have $\mathbb{P}\left(\widetilde{\xi} \in \partial T_{m}\left(B_{r}\right)\right)=0$ and hence $\mathbb{P}\left(\widetilde{\xi}_{n^{\prime}} \in T_{m}\left(B_{r}\right)\right) \rightarrow \mathbb{P}\left(\widetilde{\xi} \in T_{m}\left(B_{r}\right)\right)$, as $n^{\prime} \rightarrow \infty$. Therefore, in view of (A.7), we obtain

$$
\mathbb{P}\left(\xi \in B_{r}\right) \geq \mathbb{P}\left(\widetilde{\xi} \in T_{m}\left(B_{r}\right)\right), \quad \text { for all } m \text { and all but countably many } r>0 .
$$

Relation (2.15), however, implies that $T_{m}\left(B_{r}\right) \uparrow E$ as $m \rightarrow \infty$, which implies

$$
\mathbb{P}\left(\xi \in B_{r}\right)=1=\lim _{m \rightarrow \infty} \mathbb{P}\left(\widetilde{\xi} \in T_{m}\left(B_{r}\right)\right),
$$

for all but countably many $r$. This implies that $\mathbb{P}(\xi=0)=1$, which is a contradiction.

We have thus shown that the sequence $\left\{a_{n}\right\}$ is bounded above. One can similarly show that $\left\{a_{n}\right\}$ is bounded away from 0 . Indeed, by defining $\widetilde{a}_{n}:=1 / a_{n}$, we see that $\xi_{n}=T_{\widetilde{a}_{n}}\left(\widetilde{\xi}_{n}\right) \stackrel{d}{\rightleftarrows} \xi$. Therefore, repeating the above argument with $a_{n}, \xi_{n}$ and $\xi$ replaced by $\widetilde{a}_{n}, \widetilde{\xi}_{n}$ and $\widetilde{\xi}$, respectively, we see that $\left\{\widetilde{a}_{n} \equiv 1 / a_{n}\right\}$ is bounded.

We have thus shown that $\left\{a_{n}\right\}$ can only have positive cluster points. Suppose that $a_{n^{\prime}} \rightarrow a^{\prime}>0$ and $a_{n^{\prime \prime}} \rightarrow a^{\prime \prime}>0$, for some sub-sequences $n^{\prime}, n^{\prime \prime} \rightarrow \infty$. Since the space $\left(\mathbb{X}, d_{\mathbb{X}}\right)$ is separable, by the Skorokhod-Dudley representation (cf. Theorem 3.30 of Kallenberg, 1997), on a suitable probability space we can define $\xi^{*}$ and $\xi_{n}^{*}$ such that

$$
\xi_{n}^{*} \stackrel{d}{=} \xi_{n}, \quad \xi^{*} \stackrel{d}{=} \xi, \quad \text { and } \quad \xi_{n}^{*} \rightarrow \xi^{*}, \text { almost surely. }
$$

Thus, the continuity property (iii) in Definition 2.7 , implies that

$$
T_{a_{n^{\prime}}}\left(\xi_{n^{\prime}}^{*}\right) \rightarrow T_{a^{\prime}}\left(\xi^{*}\right) \quad \text { and } \quad T_{a_{n^{\prime \prime}}}\left(\xi_{n^{\prime \prime}}^{*}\right) \rightarrow T_{a^{\prime \prime}}\left(\xi^{*}\right),
$$

almost surely. Since also $T_{a_{n}}\left(\xi_{n}^{*}\right) \stackrel{d}{=} T_{a_{n}}\left(\xi_{n}\right) \stackrel{d}{\rightarrow} \widetilde{\xi}$, and $\xi \stackrel{d}{=} \xi^{*}$, we obtain

$$
T_{a^{\prime}}(\xi) \stackrel{d}{=} \widetilde{\xi} \stackrel{d}{=} T_{a^{\prime \prime}}(\xi) .
$$


By part (i), this is only possible if $a^{\prime}=a^{\prime \prime}$. We have thus shown that the sequence $\left\{a_{n}\right\}$ has a unique cluster point $a=a^{\prime}=a^{\prime \prime}>0$ and in view of (A.8), that $T_{a}(\xi) \stackrel{d}{=} \widetilde{\xi}$.

Part (iii). Suppose that $\limsup _{n \rightarrow \infty} a_{n}>0$, i.e., for some subsequence $n^{\prime} \rightarrow \infty$, we have $a_{n^{\prime}} \geq \epsilon_{0}>0$, for all $n^{\prime}$. Then, in view of (2.17), for all $r>0$, we have

$$
\mathbb{P}\left(T_{\epsilon_{0}}(\xi) \in B_{r}^{c}\right) \leq \mathbb{P}\left(T_{a_{n^{\prime}}}(\xi) \in B_{r}^{c}\right) .
$$

Since $T_{a_{n^{\prime}}}(\xi) \stackrel{d}{\rightarrow} 0$, the right-hand side vanishes, as $n^{\prime} \rightarrow \infty$. On the other hand, since $\xi$ is nonzero, so is $T_{\epsilon_{0}}(\xi)$ and the left-hand side is positive for sufficiently small $r>0$. This contradiction yields $\lim \sup _{n \rightarrow \infty} a_{n}=0$.

The next result is used in the proof of Corollary 3.10, given below.

Lemma A.7. Let $X:=\left\{X(\lambda), \lambda \in \Lambda_{k}\right\}$ and $X_{n}:=\left\{X_{n}(\lambda), \lambda \in \Lambda_{k}\right\}$ be random fields in $S_{c}\left(\Lambda_{k}, \mathbb{V}\right)$ such that $X_{n} \stackrel{d}{\rightarrow} X$. Then for any sequences $v_{n} \rightarrow 0$ and $r_{n} \rightarrow 1$, we have

$$
\left\{X_{n}\left(v_{n}+r_{n} \cdot \lambda\right), \lambda \in \Lambda_{k}\right\} \stackrel{d}{\longrightarrow}\left\{X(\lambda), \lambda \in \Lambda_{k}\right\} .
$$

Proof. Let $Y_{n}(\lambda)=X_{n}\left(v_{n}+r_{n} \cdot \lambda\right)$ and $\breve{Y}_{n}=Y_{n}\left(\lambda_{t}\right)$. By Proposition 2.3, conditions (i) and (ii) hold for $\breve{X}_{n}$. We need to show that they also hold for $\breve{Y}_{n}$. The proof for (i) is an easy application of the Skorokhod-Dudley representation Theorem 3.30 on page 56 in Kallenberg (1997). We focus on proving (ii).

First consider the relationship between $\breve{X}_{n}$ and $\breve{Y}_{n}$. It follows that

$$
\breve{Y}_{n}(t)=X_{n}\left(v_{n}+r_{n} \cdot \lambda_{t}\right)=\int \breve{X}_{n}\left(v_{n}+r_{n} u\right) \lambda_{t}(d u),
$$

where one can write

$$
\lambda_{t}(d u)=\sum_{i=1}^{M_{k}+1} c_{i}(t) \delta_{s_{i}(t)}(d u)
$$

for some continuous functions $c_{i}(t) \in \mathbb{C}$ and $s_{i}(t) \in \mathbb{R}^{d}, i=1, \ldots, M_{k}+1$ (recall (2.7)).

Since $\breve{X}_{n}(t)=X_{n}\left(\lambda_{t}\right)$, for any $K$ and $n$ large enough,

$$
\begin{aligned}
\sup _{\|t\|_{2} \leq K} d_{\mathbb{V}}\left(\breve{Y}_{n}(t), \breve{X}_{n}(t)\right) & \leq \sup _{\|t\|_{2} \leq K} \sum_{i=1}^{M_{k}+1} d_{\mathbb{V}}\left(c_{i}(t) \breve{X}_{n}\left(v_{n}+r_{n} s_{i}(t)\right), c_{i}(t) \breve{X}_{n}\left(s_{i}(t)\right)\right) \\
& \leq\left(M_{k}+1\right) \sup _{\substack{\|s\|,\|t\|\left\|2 K \\
|c| \leq C_{K},\right\| s-t \| \leq \delta_{n}}} d_{\mathbb{V}}\left(c \breve{X}_{n}(s), c \breve{X}_{n}(t)\right),
\end{aligned}
$$

where $\delta_{n}:=\left|r_{n}-1\right| S_{K}+\left|v_{n}\right| \rightarrow 0$ with

$$
S_{K}:=\sup _{\substack{\|t t\| K \\ i=1, \ldots, M_{k}+1}}\left\|s_{i}(t)\right\| \quad \text { and } \quad C_{K}:=\sup _{\substack{\| t t \leq K K \\ i=1, \ldots, M_{k}+1}}\left|c_{i}(t)\right| .
$$

Therefore, for any $\eta, \epsilon>0$, by (ii) in Proposition 2.3 there exists $n$ large enough such that, $\mathbb{P}\left(A_{n}<\eta\right)>1-\epsilon$, where

$$
A_{n}:=\sup _{\substack{\|s\|\|\| t\|\| 2 K \\\|s-t\| \leq \leq \delta_{n}}} d_{\mathbb{V}}\left(\breve{X}_{n}(s), \breve{X}_{n}(t)\right) .
$$

Then according to (2.3), (2.4) and (A.9), we have on the event $\left\{A_{n}<\eta\right\}$, that

$$
\sup _{\|t\| \leq K} d_{\mathbb{V}}\left(\breve{Y}_{n}(t), \breve{X}_{n}(t)\right) \leq\left(M_{k}+1\right) f_{C_{K}}(\eta),
$$


where for any $C_{K}>0, f_{C_{K}}(\eta):=\sup _{d_{\mathrm{V}}(x, y)<\eta,|c|<C_{K}} d_{\mathbb{V}}(c x, c y) \rightarrow 0$ as $\eta \rightarrow 0$. Thus, on $\left\{A_{n}<\eta\right\}$, we have

$$
\begin{aligned}
\sup _{\substack{\|s\|,\|t\| \leq K \\
\|s-t\| \leq \delta_{n}}} d_{\mathbb{V}}\left(\breve{Y}_{n}(s), \breve{Y}_{n}(t)\right) & \leq 2 \sup _{\|t\| \leq K} d_{\mathbb{V}}\left(\breve{Y}_{n}(t), \breve{X}_{n}(t)\right)+\sup _{\substack{\|s\|,\|t\| \leq K \\
\|s-t\| \leq \delta_{n}}} d_{\mathbb{V}}\left(\breve{X}_{n}(s), \breve{X}_{n}(t)\right) \\
& \leq 2\left(M_{k}+1\right) f_{C_{K}}(\eta)+\eta .
\end{aligned}
$$

Thus, the second condition of Proposition 2.3 for $\breve{Y}_{n}$ follows.

Proof of Corollary 3.10: Consider the context of the proof of Theorem 3.8. When $T_{s, c}=T_{c}, c>0$, for a fixed scaling action $T_{c}$. Relation (3.19) becomes

$$
\begin{aligned}
\xi_{n} & =\left\{T_{c_{n}\left(s_{n}\right)} \circ T_{c_{n}(s)}^{-1} \circ T_{c_{n}(s)} X\left(s,(1 / n) \cdot\left(w_{n}+\lambda\right)\right), \lambda \in \Lambda_{k}\right\} \\
& =: T_{c_{n}\left(s_{n}\right) / c_{n}(s)} \widetilde{\xi}_{n} .
\end{aligned}
$$

where $c_{n}(s):=c(s, 1 / n)$, and we used the fact that $T_{c_{n}(s)}^{-1}=T_{1 / c_{n}(s)}$.

Relation (3.18) implies that

$$
\xi_{n} \stackrel{d}{\longrightarrow} \xi:=\left\{Y_{s}(\lambda), \lambda \in \Lambda_{k}\right\} .
$$

On the other hand, by Lemma A.7, we have

$$
\widetilde{\xi}_{n}=\left\{T_{c_{n}(s)} X\left(s,(1 / n) \cdot\left(w_{n}+\lambda\right)\right), \lambda \in \Lambda_{k}\right\} \stackrel{d}{\longrightarrow} \widetilde{\xi}:=\left\{Y_{s}(w+\lambda), \lambda \in \Lambda_{k}\right\} .
$$

Thus, in view (A.10), we have $\widetilde{\xi}_{n}=T_{c_{n}(s) / c_{n}\left(s_{n}\right)} \xi_{n} \stackrel{d}{\rightarrow} \widetilde{\xi}$ and since both $\xi$ and $\widetilde{\xi}$ are non-zero, Lemma A.6 implies that

$$
\frac{c_{n}(s)}{c_{n}\left(s_{n}\right)} \equiv \frac{c_{n}(s)}{c_{n}\left(s+w_{n} / n\right)} \rightarrow a_{s}\left(\left\{w_{n}\right\}\right)>0 .
$$

One can verify that the limit $a_{s}\left(\left\{w_{n}\right\}\right)$ is independent of the choice of the sequence $\left\{w_{n}\right\}$. Indeed, if there exists another $w_{n}^{\prime} \rightarrow w$ and $s+w_{n}^{\prime} / n \in K_{\epsilon}$ then we will have

$$
\left\{Y_{s}(w+\lambda), \lambda \in \Lambda_{k}\right\} \stackrel{d}{=}\left\{T_{a_{s}\left(\left\{w_{n}\right\}\right)}\left(Y_{s}(\lambda)\right), \lambda \in \Lambda_{k}\right\} \stackrel{d}{=}\left\{T_{a_{s}\left(\left\{w_{n}^{\prime}\right\}\right)}\left(Y_{s}(\lambda)\right), \lambda \in \Lambda_{k}\right\},
$$

which shows $a_{s}\left(\left\{w_{n}\right\}\right)=a_{s}\left(\left\{w_{n}^{\prime}\right\}\right)$ by (i) of Lemma A.6. Thus, we can just use the notation $a_{s}(w)$ and we have

$$
\left\{Y_{s}(w+\lambda), \lambda \in \Lambda_{k}\right\} \stackrel{d}{=}\left\{T_{a_{s}(w)} Y_{s}(\lambda), \lambda \in \Lambda_{k}\right\} .
$$

To prove that (3.16) holds, or equivalently $T_{c_{n}(s) / c_{n}\left(s_{n}\right)} \rightarrow T_{1} \equiv \mathrm{I}$, we only need to verify $a_{s}(w)=1$, which we do next. By (A.11), it is easy to see that $a_{s}(0)=1$ and $a_{s}(w+u)=a_{s}(w) a_{s}(u)$. By Theorem 3.4, there is a positive scalar $\alpha=\alpha(s)>0$, such that

$$
\left\{Y_{s}(r \cdot \lambda), \lambda \in \Lambda_{k}\right\} \stackrel{d}{=}\left\{T_{r^{\alpha}}\left(Y_{s}(\lambda)\right), \lambda \in \Lambda_{k}\right\}, \text { for all } r>0 .
$$

Consider $Y_{s}(r \cdot(w+\lambda))$ with $r \in \mathbb{N}$. On one hand, (A.11) and (A.12) imply that

$$
\left\{Y_{s}(r \cdot(w+\lambda)), \lambda \in \Lambda_{k}\right\} \stackrel{d}{=}\left\{T_{r^{\alpha}}\left(Y_{s}(w+\lambda)\right), \lambda \in \Lambda_{k}\right\} \stackrel{d}{=}\left\{T_{r^{\alpha} a_{s}(w)}\left(Y_{s}(\lambda)\right), \lambda \in \Lambda_{k}\right\} .
$$

On the other hand, since $a_{s}(r w)=a_{s}^{r}(w)$, for all $r \in \mathbb{N}$, viewing $Y_{s}(r \cdot(w+\lambda))$ as $Y_{s}(r w+r \cdot \lambda)$, by (A.11), we have

$$
\left\{Y_{s}(r \cdot(w+\lambda)), \lambda \in \Lambda_{k}\right\} \stackrel{d}{=}\left\{T_{a_{s}(r w)}\left(Y_{s}(r \cdot \lambda)\right), \lambda \in \Lambda_{k}\right\} \stackrel{d}{=}\left\{T_{a_{s}^{r}(w) r^{\alpha}}\left(Y_{s}(\lambda)\right), \lambda \in \Lambda_{k}\right\} .
$$

Thus, by Lemma A.6 applied to (A.13) and (A.14), we obtain $a_{s}(w)=a_{s}^{r}(w)$ for all $r \in \mathbb{N}$, which, since $a_{s}(w)>0$, implies $a_{s}(w)=1$. 


\section{A.4 Supplemental background and some proofs for Section 4}

In Bochner's Theorem, we need the notion of integration of a $\mathbb{C}$-valued function on $\mathbb{R}^{d}$ with respect to a finite $\mathbb{T}_{+}$-valued measure. A finite $\mathbb{T}_{+}$-valued measure is a mapping $\mu: \mathcal{B}\left(\mathbb{R}^{d}\right) \rightarrow \mathbb{T}_{+}$from the class of Borel sets in $\mathbb{R}^{d}$ to $\mathbb{T}_{+}$that is countably additive. Notice that this readily implies that $\mu(\emptyset)=0$; that $\mu$ is monotone, i.e., $\mu(A) \leq \mu(B)$ as positive operators for $A \subset B$; and that $\mu$ is finite since $\mu\left(\mathbb{R}^{d}\right) \in \mathbb{T}_{+}$. This notion can be developed along the line of ordinary Lebesgue integral, making use of the completeness of T. We will provide a brief outline below and leave the details to Section S.3 in Shen et al. (2020). (The construction naturally extends to the case of $\sigma$-finite $\mathbb{T}_{+}$-valued measures.)

Let $\mu$ be a finite $\mathbb{T}_{+}$-valued measure. We follow the development of ordinary Lebesgue integration:

(i) For any real nonnegative simple function $f=\sum_{i=1}^{k} c_{i} I_{A_{i}}$, define $\int f d \mu=$ $\sum_{i=1}^{k} c_{i} \mu\left(A_{i}\right)$.

(ii) For nonnegative measurable functions $f$, let

$$
\int f d \mu=\lim _{n \rightarrow \infty} \int f_{n} d \mu
$$

in $\left(\mathbb{T},\|\cdot,\|_{\text {tr }}\right)$ where $\left\{f_{n}\right\}$ is any sequence of simple functions such that

(a) $f_{n} \leq f_{n+1}$,

(b) $f_{n}(x) \uparrow f(x)$ for all $x$,

(c) $\int f_{n} d \mu \leq \mathcal{B}$ for all $n$ and some fixed $\mathcal{B} \in \mathbb{T}_{+}$.

The existence of $\left\{f_{n}\right\}$ satisfying (a) and (b) for any given nonnonegative measurable $f$ follows from standard measure theory. However, we need the extra condition (c) (along with the completeness of $\mathbb{T}_{+}$) to ensure that the limit on rhs of (A.15) exists and does not depend on the choice of $\left\{f_{n}\right\}$. Clearly, (c) is automatically fulfilled if $f$ is bounded.

(iii) For a general real measurable $f$, let

$$
\int f d \mu=\int f_{+} d \mu-\int f_{-} d \mu
$$

provided both terms on the right is finite. For a general complex $f$, let

$$
\int f d \mu=\int f_{\mathrm{re}} d \mu+\dot{\mathrm{i}} \int f_{\mathrm{im}} d \mu
$$

where $f_{\mathrm{re}}, f_{\mathrm{im}}$ be the real and imaginary parts, respectively.

It is immediate that $\|\mu\|_{\operatorname{tr}}(A):=\|\mu(A)\|_{\operatorname{tr}}, A \in \mathcal{B}\left(\mathbb{R}^{d}\right)$ defines a finite Borel measure referred to as to the trace measure of $\mu$. The following useful integrability criterion is straightforward.

Proposition A.8. The integral $\int_{\mathbb{R}^{d}} f d \mu \in \mathbb{T}$ is well-defined, for all $f \in L^{1}\left(\mathbb{R}^{d},\|\mu\|_{\mathrm{tr}}\right)$, and

$$
\int_{\mathbb{R}^{d}} f(x)\|\mu\|_{\operatorname{tr}}(d x)=\operatorname{trace}\left(\int_{\mathbb{R}^{d}} f(x) \mu(d x)\right) .
$$

The integral can be readily extended to $\sigma$-finite $\mathbb{T}$-valued signed measures. One can also naturally consider finite signed TT-valued measures, namely $\mu=\mu_{+}-\mu_{-}$ where $\mu_{+}, \mu_{-}$are both finite $\mathbb{T}_{+}$-valued measures, by $\int f d \mu:=\int f d \mu_{+}-\int f d \mu_{-}$. In this case, the trace measure of $\mu$ is the (scalar) signed measure defined as $\|\mu\|_{\operatorname{tr}}(\cdot):=$ $\left\|\mu_{+}\right\|_{\operatorname{tr}}(\cdot)-\left\|\mu_{-}\right\|_{\operatorname{tr}}(\cdot)$. 
Next we turn to the stochastic integral in Cramér's representation. Recall that $\mathbb{L}^{2}(\Omega)$ is the $L^{2}$-space of all $\mathbb{V}$-valued random elements $\eta$ on the probability space $(\Omega, \mathcal{F}, \mathbb{P})$ with $\mathbb{E}\|\eta\|^{2}<\infty$, equipped with the inner product

$$
\left\langle\eta_{1}, \eta_{2}\right\rangle_{\Omega}:=\mathbb{E}\left\langle\eta_{1}, \eta_{2}\right\rangle .
$$

We briefly discuss the properties of the orthogonal random measure $\xi=\{\xi(A), A \in$ $\left.\mathcal{B}\left(\mathbb{R}^{d}\right)\right\}$ in Definition 4.6. Condition (ii) therein readily implies the orthogonality as well as finite additivity of the measure $\xi$. Indeed,

$$
\mathbb{E}[\xi(A) \otimes \xi(B)]=\mu(\emptyset)=0, \quad \text { for all disjoint } A, B \in \mathcal{B}\left(\mathbb{R}^{d}\right),
$$

where 0 stands for the zero operator in $\mathbb{T}$. This implies in particular that $\langle\xi(A), \xi(B)\rangle_{\Omega}=0$ (cf Remark A.9). We have, moreover, that for all (orthogonal) projection operators $\Pi: \mathbb{V} \rightarrow \mathbb{V}$, the random measure $\eta(\cdot):=\Pi \xi(\cdot)$ is also an orthogonal measure with control measure $\Pi \mu(\cdot) \Pi$.

Now, to show finite additivity, observe that for disjoint Borel sets $A$ and $B$,

$$
\begin{aligned}
\mathbb{E} \| \xi(A & \cup B)-\xi(A)-\xi(B) \|^{2} \\
& =\mathbb{E} \operatorname{trace}((\xi(A \cup B)-\xi(A)-\xi(B)) \otimes(\xi(A \cup B)-\xi(A)-\xi(B))) \\
& =\operatorname{trace}(\mu(A \cup B)-\mu(A)-\mu(B))=0,
\end{aligned}
$$

by the fact that the $\mathbb{E}$ and trace operators can be exchanged and the finite additivity of $\mu$. This shows $\xi(A \cup B)=\xi(A)+\xi(B)$, almost surely.

Finally, Condition (i) in Definition 4.6 along with the established finite additivity implies the $\sigma$-additivity of $\xi$, in the sense that for any sequence of pairwise disjoint $A_{n} \in \mathcal{B}\left(\mathbb{R}^{d}\right), n=1,2, \cdots$, we have

$$
\xi\left(\bigcup_{n=1}^{\infty} A_{n}\right)=\sum_{n=1}^{\infty} \xi\left(A_{n}\right), \quad \text { almost surely, }
$$

where the series on the rhs stands for the limit of $\sum_{n=1}^{N} \xi\left(A_{n}\right)$ as $N \rightarrow \infty$ in $\mathbb{L}^{2}(\Omega)$.

Remark A.9. Observe that for random vectors $X, Y \in \mathbb{L}^{2}(\Omega)$, we have that

$$
\mathbb{E}[X \otimes Y]=0 \quad \text { implies } \quad\langle X, Y\rangle_{\Omega}=0,
$$

but the converse implication is not always true. Thus, the orthogonality condition in (A.16) is stronger than requiring simply $\langle\xi(A), \xi(B)\rangle_{\Omega}=0$.

Introduce the finite Borel scalar measure

$$
\|\mu\|_{\operatorname{tr}}(A):=\operatorname{trace}(\mu(A)) \equiv\|\mu(A)\|_{\operatorname{tr}}, \quad A \in \mathcal{B}\left(\mathbb{R}^{d}\right) .
$$

It is easy to verify (cf. Lemma S.2.2 in Shen et al., 2020) that, for all $A \in \mathcal{B}\left(\mathbb{R}^{d}\right)$,

$$
\|\xi(A)\|_{\Omega}^{2}=\|\mu(A)\|_{\mathrm{tr}}=\|\mu\|_{\mathrm{tr}}(A) .
$$

Next, for an orthogonal random measure $\xi$ with control measure $\mu$, we sketch the construction of the stochastic integral $\mathcal{I}_{\xi} f:=\int_{\mathbb{R}^{d}} f(t) \xi(d t)$ defined for all functions $f \in \mathbb{L}^{2}\left(\mathbb{R}^{d},\|\mu\|_{\text {tr }}\right)$, i.e., all measurable $f: \mathbb{R}^{d} \rightarrow \mathbb{C}$ with $\int_{\mathbb{R}^{d}}|f(t)|^{2}\|\mu\|_{\operatorname{tr}}(d t)<\infty$. For any simple function $\psi(x)=\sum_{j=1}^{n} c_{j} I_{A_{j}}(x)$, where $A_{j} \in \mathcal{B}\left(\mathbb{R}^{d}\right), A_{j} \cap A_{j^{\prime}}=\emptyset$ when $j \neq j^{\prime}$, define the integral

$$
\mathcal{I}_{\xi}(\psi)=\sum_{j=1}^{n} c_{j} \xi\left(A_{j}\right)
$$


The integral $\mathcal{I}_{\xi}(\psi)$ takes values in $\mathbb{L}_{\xi}^{2}(\Omega)$, defined as the closure of

$$
\operatorname{span}(\xi):=\left\{\sum_{j=1}^{n} c_{i} \xi\left(A_{j}\right), c_{j} \in \mathbb{C}, A_{j} \in \mathcal{B}\left(\mathbb{R}^{d}\right), n=1,2, \ldots\right\}
$$

in $\mathbb{L}^{2}(\Omega)$. Property (ii) in Definition 4.6 entails

$$
\left\|\mathcal{I}_{\xi}(\psi)\right\|_{\Omega}^{2}=\int_{\mathbb{R}^{d}}|\psi(x)|^{2}\|\mu\|_{\operatorname{tr}}(d x) .
$$

Therefore, $\mathcal{I}_{\xi}$ is an isometric linear mapping between the class of simple functions in the $L^{2}$-space $\mathbb{L}^{2}\left(\mathbb{R}^{d},\|\mu\|_{\text {tr }}\right)$ and the Hilbert space $\mathbb{L}_{\xi}^{2}(\Omega)$.

As the class of simple functions is dense in $\mathbb{L}^{2}\left(\mathbb{R}^{d},\|\mu\|_{\text {tr }}\right)$ and the integrals $\mathcal{I}_{\xi}(\psi)$ are dense in $\mathbb{L}_{\xi}^{2}(\Omega)$, the linear operator $\mathcal{I}_{\xi}$ can be uniquely extended to an isometric linear mapping between $\mathbb{L}^{2}\left(\mathbb{R}^{d},\|\mu\|_{\text {tr }}\right)$ and $\mathbb{L}_{\xi}^{2}(\Omega)$. This completes the construction of the stochastic integral

$$
\int_{\mathbb{R}^{d}} f(x) \xi(d x):=\mathcal{I}_{\xi}(f), \quad f \in \mathbb{L}^{2}\left(\mathbb{R}^{d},\|\mu\|_{\mathrm{tr}}\right) .
$$

Observe, moreover that for all $f, g \in \mathbb{L}^{2}\left(\mathbb{R}^{d},\|\mu\|_{\text {tr }}\right)$, we have

$$
\mathbb{E}\left[\mathcal{I}_{\xi}(f) \otimes \mathcal{I}_{\xi}(g)\right]=\int_{\mathbb{R}^{d}} f(x) \bar{g}(x) \mu(d x),
$$

where the last integral is well-defined in view of Proposition A.8.

Proof of Proposition 4.18: Part (i): If $Y$ is a real $\operatorname{IRF}_{k}$ then $Y$ is also a real $\operatorname{IRF}_{k+1}$ with trivial polynomial spectral characteristic. Indeed, since for all $\lambda \in \Lambda_{k+1}$, we have $\partial_{j_{1}, \cdots, j_{d}}^{k+1} \widehat{\lambda}(0)=0$, Relation (4.16) becomes

$$
Y(\lambda)=\int_{\mathbb{R}^{d}} \frac{\widehat{\lambda}(u)}{1 \wedge\|u\|^{k+1}} \xi(d u)=: \int_{\mathbb{R}^{d}} \frac{\widehat{\lambda}(u)}{1 \wedge\|u\|^{k+2}} \eta(d u),
$$

where $\eta(d u):=\left(1 \wedge\|u\|^{k+2}\right)\left(1 \wedge\|u\|^{k+1}\right)^{-1} \xi(d u)$. By taking $\lambda \in \Lambda_{k+1}(\mathbb{R})$, since $Y(\lambda)=\overline{Y(\lambda)}$ and $\overline{\hat{\lambda}(u)}=\widehat{\lambda}(-u)$, we obtain that

$$
Y(\lambda)=\int_{\mathbb{R}^{d}} \frac{\widehat{\lambda}(u)}{1 \wedge\|u\|^{k+2}} \overline{\eta(-d u)}, \quad \lambda \in \Lambda_{k+1}(\mathbb{R}) .
$$

By (4.18), the last relation continues to hold for all complex $\lambda \in \Lambda_{k+1}$. Hence, appealing to the uniqueness of the representation (4.16) of $Y$ viewed as an $\mathrm{IRF}_{k+1}$ (with $k$ replaced by $k+1$ and $\xi$ by $\eta$ ), we obtain

$$
\eta(d u)=\overline{\eta(-d u)}, \quad \text { almost surely, }
$$

or equivalently $\xi(d u)=\overline{\xi(-d u)}$, a.s., which shows that the orthogonal measure $\xi$ is Hermitian.

The fact that $\xi$ is Hermitian and $Y$ a real $\operatorname{IRF}_{k}$, imply that for all $\lambda \in \Lambda_{k}(\mathbb{R})$,

$$
Y(\lambda)-\int_{\mathbb{R}^{d}} \frac{\widehat{\lambda}(u)}{1 \wedge\|u\|^{k+1}} \xi(d u)=\sum_{j_{1}, \cdots, j_{d}} \partial_{j_{1}, \cdots, j_{d}}^{k+1} \widehat{\lambda}(0) Z_{j_{1}, \cdots, j_{d}} \quad \text { is real. }
$$

By taking suitable real $\lambda^{\prime}$ 's for which $\partial_{j_{1}, \cdots, j_{d}}^{k+1} \widehat{\lambda}(0)$ vanish for all but each one term in the sum, we obtain that all (i) $)^{k+1} Z_{j_{1}, \cdots, j_{d}}$ must be real. 
Conversely, if $\xi$ is Hermitian and the $(\mathfrak{i})^{k+1} Z_{j_{1}, \cdots, j_{d}}$ 's are real, it is immediate that $Y(\lambda)$ is real for all $\lambda \in \Lambda_{k}(\mathbb{R})$. This completes the proof of part (i). Part (ii) is an immediate consequence of (i).

Part (iii): One can define $Y$ as in (4.18), where all (i) ${ }^{k+1} Z_{j_{1}, \cdots, j_{d}}$ 's are real and $\xi$ Hermitian. By part (i), this entails that $Y$ is a real $\operatorname{IRF}_{k}$. Suppose now that $\widetilde{Y}(\lambda)=$ $a Y(\lambda)+\dot{\mathrm{i}} b Y^{\prime}(\lambda)$ and observe that

$$
\begin{aligned}
\mathcal{C}_{\widetilde{Y}}(\lambda, \mu) & =a^{2} \mathbb{E}[Y(\lambda) \otimes Y(\mu)]+b^{2} \mathbb{E}\left[Y^{\prime}(\lambda) \otimes Y^{\prime}(\mu)\right]+a b \dot{\mathrm{n}}\left[\mathbb{E} Y^{\prime}(\lambda) \otimes Y(\mu)-\mathbb{E} Y(\lambda) \otimes Y^{\prime}(\mu)\right] \\
& =\mathbb{E}[Y(\lambda) \otimes Y(\mu)]=\mathcal{C}_{Y}(\lambda, \mu),
\end{aligned}
$$

since $a^{2}+b^{2}=1$ and the cross-covariance terms cancel. This shows that $\tilde{Y}$ and $Y$ have the same covariance structure.

Lemma A.10. Let $T(x, y)$ be a bivariate mapping from $\mathbb{D} \times \mathbb{D}$ to $\mathbb{F}$, where both $\mathbb{D}$ and $\mathbb{F}$ are linear spaces over $\mathbb{C}$. Assume that $T$ is sesquilinear form with $T(c x, y)=c T(x, y)$ and $T(x, c y)=\bar{c} T(x, y), x, y \in D, c \in \mathbb{C}$. Then, for $x, y \in \mathbb{D}$,

$$
T(x, y)=\frac{\dot{\mathrm{i}}-1}{2}(T(x, x)+T(y, y))+\frac{1}{2} T(x+y, x+y)-\frac{\dot{\mathbb{i}}}{2} T(\dot{\mathrm{i}} x+y, \dot{\mathrm{i}} x+y) .
$$

Proof. The proof is trivial by noticing the identities:

$$
\begin{aligned}
T(x+y, x+y) & =T(x, x)+T(y, y)+T(x, y)+T(y, x), \\
T(\dot{\mathfrak{i}} x+y, \dot{\mathrm{i}} x+y) & =T(x, x)+T(y, y)+\dot{\mathrm{i}} T(x, y)-\dot{\mathrm{i}} T(y, x) .
\end{aligned}
$$

\section{A.5 Proofs for Section 5.1}

We start with an auxiliary result needed for the proof of Theorem 5.3 below.

Lemma A.11. For any $\mathcal{A} \in \mathbb{T}$ and any bounded linear operator $\mathcal{H}$, define $f(c):=$ $c^{-\mathcal{H}} \mathcal{A} c^{-\mathcal{H}^{*}}, c>0$. The function $f:(0, \infty) \rightarrow \mathbb{T}$ is continuously Fréchet differentiable in $\left(\mathbb{T},\|\cdot\|_{\text {tr }}\right)$ with derivative

$$
f^{\prime}(c)=-c^{-1}\left(\mathcal{H} f(c)+f(c) \mathcal{H}^{*}\right)=-c^{-1} c^{-\mathcal{H}}\left(\mathcal{H} \mathcal{A}+\mathcal{A} \mathcal{H}^{*}\right) c^{-\mathcal{H}^{*}}, c>0 .
$$

That is,

$$
\left\|f(c+h)-f(c)-f^{\prime}(c) h\right\|_{\operatorname{tr}}=o(h), \quad \text { as } h \rightarrow 0 .
$$

Proof. Observe that $(c+h)^{-\mathcal{H}}-c^{-\mathcal{H}}=\sum_{n=1}^{\infty}(-\mathcal{H})^{n}\left[(\log (c+h))^{n}-(\log c)^{n}\right] / n$ !. Applying the mean value theorem to the terms $(\log (c+h))^{n}-(\log c)^{n}$, and using the triangle inequality for $\|\cdot\|_{\mathrm{op}}$, one can show that for all $c>0$,

$$
\left\|(c+h)^{-\mathcal{H}}-c^{-\mathcal{H}}-c^{-\mathcal{H}}(-\mathcal{H}) c^{-1} h\right\|_{\mathrm{op}}=o(h), \quad \text { as } h \rightarrow 0 .
$$

That is, $c \mapsto c^{-\mathcal{H}}$ and similarly $c \mapsto c^{-\mathcal{H}^{*}}$ are Fréchet differentiable in the Hilbert space V.

On the other hand, by Proposition IV.5.4 on page 62 of Gohberg et al. (2000), for any two bounded operators $\mathcal{B}$ and $\mathcal{C}$ and a trace class operator $\mathcal{A} \in \mathbb{T}$, we have that $\mathcal{B} \mathcal{A C} \in \mathbb{T}$ and moreover

$$
\|\mathcal{B} \mathcal{A C}\|_{\text {tr }} \leq\|\mathcal{B}\|_{\text {op }}\|\mathcal{A}\|_{\text {tr }}\|\mathcal{C}\|_{\text {op }}
$$

This inequality can be used to show that the Fréchet differentiability of $c \mapsto c^{-\mathcal{H}}$ and $c \mapsto c^{-\mathcal{H}^{*}}$ in the operator norm induced by the Hilbert space norm in $\mathbb{V}$ entails the Fréchet differentiability in trace-norm. 
Indeed, for all $c>0$ and $h>-c$, we have

$$
\begin{aligned}
f(c+h)-f(c) & =(c+h)^{-\mathcal{H}} \mathcal{A}(c+h)^{-\mathcal{H}^{*}}-c^{-\mathcal{H}} \mathcal{A} c^{-\mathcal{H}^{*}} \\
& =\left((c+h)^{-\mathcal{H}}-c^{-\mathcal{H}}\right) \mathcal{A}(c+h)^{-\mathcal{H}^{*}}+c^{-\mathcal{H}} \mathcal{A}\left((c+h)^{-\mathcal{H}^{*}}-c^{-\mathcal{H}^{*}}\right) .
\end{aligned}
$$

Now, in view of (A.18) and using the inequality (A.19), we obtain

$$
\left\|f(c+h)-f(c)-h c^{-\mathcal{H}-1}(-\mathcal{H}) \mathcal{A}(c+h)^{-\mathcal{H}^{*}}-h(c+h)^{-\mathcal{H}} \mathcal{A}\left(-\mathcal{H}^{*}\right) c^{-\mathcal{H}^{*}-1}\right\|_{\text {tr }}=o(h),
$$

as $h \rightarrow 0$. The continuity of $c \mapsto c^{-\mathcal{H}}$ and $c \mapsto c^{-\mathcal{H}^{*}}$ in the operator norm and another application of (A.19) entails (A.17).

Proof of Theorem 5.3: Fix $c>0$ and define the rescaled $\operatorname{IRF}_{k}$ process $\tilde{Y}(\lambda):=$ $c^{-\mathcal{H}} Y(c \cdot \lambda), \lambda \in \Lambda_{k}$. Observe that $Y$ is covariance $\mathcal{H}$-self-similar if and only if $\widetilde{Y}$ and $Y$ have the same covariance structure or, equivalently, the same spectral characteristics $(\chi, \mathcal{Q})$.

In view of (4.14), for all $\lambda, \mu \in \Lambda_{k}$, we have

$$
\begin{aligned}
\mathcal{C}_{\widetilde{Y}}(\lambda, \mu) & =\mathbb{E}[\tilde{Y}(\lambda) \otimes \tilde{Y}(\mu)]=c^{-\mathcal{H}} \mathbb{E}[Y(c \cdot \lambda) \otimes Y(c \cdot \mu)] c^{-\mathcal{H}^{*}} \\
& =\int_{\mathbb{R}^{d}} \widehat{c \cdot \lambda}(u) \overline{\widehat{c \cdot \mu}}(u) c^{-\mathcal{H}} \chi_{k}(d u) c^{-\mathcal{H}^{*}}+c^{-\mathcal{H}} \mathcal{Q}(c \cdot \lambda * \widetilde{c \cdot \mu}) c^{-\mathcal{H}^{*}} \\
& =\int_{\mathbb{R}^{d}} \widehat{\lambda}(x) \overline{\widehat{\mu}}(x) c^{-\mathcal{H}} \chi_{k}(d x / c) c^{-\mathcal{H}^{*}}+c^{-\mathcal{H}} c^{2 k+2} \mathcal{Q}(\lambda * \widetilde{\mu}) c^{-\mathcal{H}^{*}},
\end{aligned}
$$

where in the last relation we used that $\widehat{c \cdot \lambda}(u)=\widehat{\lambda}(c u)$, the change of variables $u:=x / c$, and the fact that $\mathcal{Q}(c \cdot \nu)=c^{2 k+2} \mathcal{Q}(\nu)$ for all $\nu \in \Lambda_{2 k+1}$.

Relation (A.20) shows that the spectral characteristics of $\widetilde{Y}$ are

$$
\left(c^{-\mathcal{H}} \chi_{k}(d x / c) c^{-\mathcal{H}^{*}}, c^{k+1-\mathcal{H}} \mathcal{Q}(\cdot) c^{k+1-\mathcal{H}^{*}}\right) .
$$

Hence, by the uniqueness of the spectral representation in (4.14), the $\mathrm{IRF}_{k}$ process $Y$ and $\widetilde{Y}$ have the same covariance structure, if and only if Relation (5.6) holds. This completes the proof of part (i).

Part (iv) is an immediate consequence of part (ii) and Theorem 4.15, where the random measure $\xi(d x) /\left(1 \wedge|x|^{k+1}\right)$ therein, is now written in polar coordinates as $W(d r d \theta)$. Thus, in the remainder of the proof we focus on establishing the disintegration formula (5.8) (part (ii)) and the uniqueness of the measure $\sigma$ (part (iii)).

Define the $\mathbb{T}$-valued set-mapping

$$
\sigma(B):=\mathcal{H} \chi_{k}((1, \infty) \times B)+\chi_{k}((1, \infty) \times B) \mathcal{H}^{*}, \quad B \in \mathcal{B}(\mathbb{S}),
$$

where in short, we write $\chi_{k}(A \times B)$ for $\chi_{k}(\{(r, \theta) \in A \times B\})$, with $A \subset(0, \infty)$ and $B \subset \mathbb{S}$. The fact that $\chi_{k}$ is a $\mathbb{T}_{+}$-valued measure readily implies $\sigma(\emptyset)=0$ and the countable additivity of $\sigma$. Note also that $\sigma$ is finite, since for all $B \in \mathcal{B}(\mathbb{S})$, by (A.19),

$$
\|\sigma(B)\|_{\text {tr }} \leq\left(\|\mathcal{H}\|_{\text {op }}+\left\|\mathcal{H}^{*}\right\|_{\text {op }}\right)\left\|\chi_{k}((1, \infty) \times \mathbb{S})\right\|_{\text {tr }}<\infty .
$$

We will argue next that $\sigma(B)$ is positive and hence it defines a $\mathbb{T}_{+}$-valued measure on S. We will also show that for all $c>0$ and $B \in \mathcal{B}(\mathbb{S})$, we have

$$
\chi_{k}((c, \infty) \times B)=\int_{c}^{\infty} u^{-\mathcal{H}} \sigma(B) u^{-\mathcal{H}^{*}} \frac{d u}{u} .
$$


This fact and a standard $\pi-\lambda$ argument then entail that (5.7) holds. Indeed, the right-hand side of (5.7) defines a $\sigma$-finite $\mathbb{T}_{+}$-valued measure, say $\widetilde{\chi}_{k}$, on $\mathcal{B}\left(\mathbb{R}^{d} \backslash\{0\}\right)$. By Relation (A.22), the measures $\chi_{k}$ and $\tilde{\chi}_{k}$ agree on the semi-ring of rectangle sets $(c, \infty) \times B$. Since the latter generates the $\sigma$-field $\mathcal{B}\left(\mathbb{R}^{d} \backslash\{0\}\right)$, by considering projections on a fixed CONS, it can be seen that the two $\mathbb{T}_{+}$-valued measures coincide.

We now prove that $\sigma$ is a finite $\mathbb{T}_{+}$-valued measure and show (A.22). In view of Lemma A.11 (above), it follows that the function

$$
f(u):=u^{-\mathcal{H}} \chi_{k}((1, \infty) \times B) u^{-\mathcal{H}^{*}} .
$$

is Fréchet continuously differentiable in $\left(\mathbb{T},\|\cdot\|_{\text {tr }}\right)$ with derivative:

$$
\begin{aligned}
f^{\prime}(u) & =-u^{-1} u^{-\mathcal{H}}\left(\mathcal{H} \chi_{k}((1, \infty) \times B)+\chi_{k}((1, \infty) \times B) \mathcal{H}^{*}\right) u^{-\mathcal{H}^{*}} \\
& =-u^{-1} u^{-\mathcal{H}} \sigma(B) u^{-\mathcal{H}^{*}}, \quad u>0 .
\end{aligned}
$$

Observe that by the operator-scaling property for $\chi_{k}$ in (5.6), we have

$$
f(u)=\chi_{k}((u, \infty) \times B), u>0 .
$$

The monotonicity of the $\mathbb{T}_{+}$-valued measure $\chi_{k}$ then implies that

$$
\chi_{k}((u, u+h] \times B)=f(u)-f(u+h) \in \mathbb{T}_{+},
$$

for all $h>0$. This shows that $-f^{\prime}(u) \in \mathbb{T}_{+}$and by setting $u=1$, we obtain $-f^{\prime}(1)=$ $\sigma(B) \in \mathbb{T}_{+}$, which shows that $\sigma(B) \in \mathbb{T}_{+}$, completing the proof that the so-defined set-mapping in (A.21) is a finite $\mathbb{T}_{+}$-valued measure.

Now, using (A.23) and a straightforward extension of the fundamental theorem of calculus to Bochner integrals, we obtain

$$
\begin{aligned}
\int_{c}^{\infty} u^{-\mathcal{H}} \sigma(B) u^{-\mathcal{H}^{*}} \frac{d u}{u} & =-\int_{c}^{\infty} f^{\prime}(u) d u=f(c) \\
& =c^{-\mathcal{H}} \chi_{k}((1, \infty) \times B) c^{-\mathcal{H}^{*}} .
\end{aligned}
$$

(Note that $f(u)=\chi_{k}((u, \infty) \times B) \downarrow 0$ as $u \uparrow \infty$.) The latter, in view of the scaling property of $\chi_{k}$, equals $\chi_{k}((c, \infty) \times B)$, completing the proof of (A.22) and part (ii).

Part (iii). We now show that $\sigma$ is uniquely determined by $\chi_{k}$, alone. By definition, we have

$$
\sigma(B)=-\left.\frac{d}{d c}\right|_{c=1} \chi_{k}((c, \infty) \times B), \quad B \in \mathcal{B}(\mathbb{S}),
$$

where the latter is interpreted as the Fréchet derivative in $(\mathbb{T},\|\cdot\|)$ of the function $c \mapsto \chi_{k}((c, \infty) \times B)$, evaluated at $c=1$. This shows that $\sigma$ is uniquely determined in terms of the measure $\chi_{k}$ and it does not depend on the choice of the exponent operator $\mathcal{H}$, which need not be unique (see e.g., Remark 5.14).

\section{A.6 Proofs for Section 5.2}

Proof of Proposition 5.16: Stationarity is immediate, provided that the stochastic integral in (5.30) is well-defined. To this end, it suffices to show that

$$
\begin{aligned}
\int_{0}^{\infty} \int_{\mathbb{S}}\left\|f_{s}(u, \theta)\right\|_{\mathrm{op}}^{2} d u\|\mu\|_{\mathrm{tr}}(d \theta) & :=\int_{0}^{\infty} \int_{\mathbb{S}}(1 \wedge u)^{2(k+1)}\left\|u^{-\mathcal{H}} \mathcal{A}(\theta)\right\|_{\mathrm{op}}^{2} u^{-1} d u\|\mu\|_{\mathrm{tr}}(d \theta) \\
& \leq \int_{\mathbb{S}}\|\mathcal{A}(\theta)\|_{\mathrm{op}}^{2}\|\mu\|_{\mathrm{tr}}(d \theta) \int_{0}^{\infty}(1 \wedge u)^{2(k+1)}\left\|u^{-\mathcal{H}}\right\|_{\mathrm{op}}^{2} u^{-1} d u .
\end{aligned}
$$


By the assumption (5.29), the first integral in (A.24) is finite. It remains to show that the second one therein is also finite. This, however, readily follows from the inequality

$$
(1 \wedge u)^{2(k+1)} u^{-1}\left\|u^{-\mathcal{H}}\right\|_{\text {op }}^{2} \leq C \times\left(1_{(0,1)}(u) u^{2 \delta-1}+1_{[1, \infty)}(u) u^{-2 \epsilon-1}\right),
$$

where in view of (5.28) and the compactness of $\Re(\operatorname{sp}(\mathcal{H})), \epsilon>0$ and $\delta>0$ are such that $\Re(\operatorname{sp}(\mathcal{H})) \subset(\epsilon, k+1-\delta)$. Relation (A.25) can be established exactly as in the proof of (5.19) using (5.28) and Riesz functional calculus. This completes the proof of (A.25) and part (i).

To prove part (ii), it suffices to establish that, for all $s, t \in B(0, M), M>0$,

$$
\mathbb{E}\left[\|X(s)-X(t)\|^{2}\right] \leq \int_{0}^{\infty} \int_{\mathbb{S}}\left\|f_{s}(u, \theta)-f_{t}(u, \theta)\right\|_{\text {op }}^{2} d u\|\mu\|_{\operatorname{tr}}(d \theta) \leq C\|s-t\|^{2 \zeta} .
$$

Indeed, consider the Gaussian $\mathbb{V}$-valued variables $\xi_{s, t}:=(X(s)-X(t)) / \sigma_{s, t}$, where $\sigma_{s, t}^{2}:=\mathbb{E}\left[\|X(s)-X(t)\|^{2}\right]$, and where by convention $\xi_{s, t}:=0$ if $\sigma_{s, t}=0$. By Corollary S.5.4 in Shen et al. (2020) (with $\theta:=1 / 4$ therein) we have that

$$
\mathbb{E}\left[\left\|\xi_{s, t}\right\|^{p}\right] \leq c_{p} \mathbb{E} \exp \left\{\left\|\xi_{s, t}\right\|^{2} / 4\right\} \leq c_{p} \sqrt{2}
$$

for all $p>0$ and some finite universal constant $c_{p}$. The last bound and Relation (A.26) can be equivalently written as

$$
\mathbb{E}\left[\|X(s)-X(t)\|^{p}\right] \leq C_{p} \sqrt{2}\|s-t\|^{p \zeta} .
$$

This, in view of Proposition 2.4, implies the existence of a $\gamma$-Hölder continuous version of $\{X(s), s \in B(0, M)\}$ for all $\gamma \in(0, \zeta-d / p)$. Taking $p$ large, we see that every $\gamma \in(0, \zeta)$ is a possible Hölder exponent. We shall continue to denote this continuous-path version of the process by $\{X(s)\}$.

Now, we turn to proving (A.26). We have

$$
\left\|f_{s}(u, \theta)-f_{t}(u, \theta)\right\|_{\mathrm{op}}^{2} \leq 2\left|e^{\mathrm{i} s} s^{\top} u \theta-e^{\mathrm{i} t^{\top} u \theta}\right|^{2}(1 \wedge u)^{2(k+1)} u^{-1}\|\mathcal{A}(\theta)\|_{\mathrm{op}}^{2}\left\|u^{-\mathcal{H}}\right\|_{\mathrm{op}}^{2} \text {. }
$$

By Relations (5.29) and (A.25), we have

$$
\begin{aligned}
I_{s, t} & :=\int_{0}^{\infty} \int_{\mathbb{S}}\left\|f_{s}(u, \theta)-f_{t}(u, \theta)\right\|_{\mathrm{op}}^{2} d u\|\mu\|_{\operatorname{tr}}(d \theta) \\
& \leq C \int_{0}^{\infty} \sup _{\theta \in \mathbb{S}}\left|e^{\mathrm{i}(s-t)^{\top} u \theta}-1\right|^{2}\left(u^{2 \delta-1} 1_{[0,1]}(u)+u^{-2 \epsilon-1} 1_{[1, \infty)}(u)\right) d u .
\end{aligned}
$$

Thus, Lemma A.12 applied with $\Delta:=(s-t)^{\top} \theta, \gamma:=2 \delta>0$ and $\epsilon:=2 \epsilon$, yields

$$
I_{s, t} \leq C\|s-t\|^{2(\epsilon \wedge 1)}\left(1+|\log (\|s-t\|)|^{2} 1_{\{\epsilon=1\}}\right) .
$$

This implies that (A.26) holds with any $\zeta<1 \wedge \epsilon$.

Part (iii). Consider the measures in (2.7) and observe that

$$
\lambda_{t}=\delta_{t}-\sum_{j} c_{j}(t) \delta_{t_{j}}
$$

where the $c_{j}(t)$ 's are polynomials in $t$ of degrees up to $k$ and the $t_{j}$ 's are some fixed points in $\mathbb{R}^{d}$. In view of Proposition 2.4, to prove (5.31) it is enough to work with

$$
\begin{aligned}
\breve{X}_{r}(t) & :=r^{-\mathcal{H}} X\left(s_{0}+r \cdot \lambda_{t}\right) \\
& =r^{-\mathcal{H}} \int_{0}^{\infty} \int_{\mathbb{S}} e^{\dot{\mathrm{i} s} s_{0}^{\top} u \theta}\left(e^{\mathrm{i} r s^{\top} u \theta}-\sum_{j} c_{j}(s) e^{\mathrm{i} r t_{j}^{\top} u \theta}\right)(1 \wedge u)^{k+1} u^{-\mathcal{H}-1 / 2} \mathcal{A}(\theta) W(d u, d \theta) \\
& =\int_{0}^{\infty} \int_{\mathbb{S}} \widehat{\lambda}_{t}(r u \theta)(1 \wedge u)^{k+1}(r u)^{-\mathcal{H}-1 / 2} \mathcal{A}(\theta) \widetilde{W}(d u, d \theta),
\end{aligned}
$$


where $\widetilde{W}(d u, d \theta):=e^{\mathrm{i} u s_{0}^{\top} \theta} r^{1 / 2} W(d u, d \theta)$. We will show that since the $\mathbb{V}$-valued Gaussian random measure $W$ is circularly symmetric and self-similar (recall (5.27)), we have

$$
\{\widetilde{W}(d u, d \theta)\} \stackrel{f d d}{=}\{W(d(r \cdot u), d \theta)\} .
$$

Indeed, since $W$ is standard Gaussian, it is not only orthogonal but also an independently scattered measure. This readily implies that $\widetilde{W}$ is also independently scattered. It is straightforward that $\widetilde{W}$ has control measure $r d u \mu(d \theta)$. It remains to show that $\widetilde{W}$ is standard, i.e., its real and imaginary components are independent and identically distributed. Indeed, for all bounded Borel $A \subset \mathbb{R}^{d} \backslash\{0\}$, in view of (5.25), we have

$$
\begin{aligned}
\widetilde{W}(A)= & \int_{A} \cos \left(\varphi_{u, \theta}\right) r^{1 / 2} W_{\mathbb{R}}(d u, d \theta)-\int_{A} \sin \left(\varphi_{u, \theta}\right) r^{1 / 2} W_{\mathbb{I}}(d u, d \theta) \\
& +\dot{\mathbb{1}}\left(\int_{A} \sin \left(\varphi_{u, \theta}\right) r^{1 / 2} W_{\mathbb{R}}(d u, d \theta)+\int_{A} \cos \left(\varphi_{u, \theta}\right) r^{1 / 2} W_{\mathrm{I}}(d u, d \theta)\right) \\
= & : \Re(\widetilde{W}(A))+\dot{\mathrm{i}} \Im(\widetilde{W}(A)),
\end{aligned}
$$

where $\varphi_{u, \theta}:=u s_{0}^{\top} \theta$. Using the fact that $W_{\mathbb{R}}$ and $W_{\mathbb{I}}$ are independent and identically distributed real orthogonal measures, we obtain that $\Re(\widetilde{W}(A))$ and $\Im(\widetilde{W}(A))$ are independent and identically distributed. This, since $\widetilde{W}$ is independently scattered and Gaussian, completes the proof of (A.29).

Therefore, by (A.29),

$$
\begin{aligned}
\left\{\breve{X}_{r}(t), t \in \mathbb{R}^{d}\right\} & \stackrel{d}{=}\left\{\int_{0}^{\infty} \int_{\mathbb{S}} \widehat{\lambda}_{t}(v \theta)(1 \wedge(v / r))^{k+1} v^{-\mathcal{H}-1 / 2} \mathcal{A}(\theta) W(d v, d \theta), t \in \mathbb{R}^{d}\right\} \\
& =:\left\{X_{r}(t), t \in \mathbb{R}^{d}\right\},
\end{aligned}
$$

where we made the change of variables $v:=r u$.

This relation readily implies that the convergence in (5.31) holds in the sense of finitedimensional distributions. Indeed, writing $\breve{Y}(t):=\int_{0}^{\infty} \int_{\mathbb{S}} \widehat{\lambda}_{t}(v \theta) v^{-\mathcal{H}-1 / 2} \mathcal{A}(\theta) W(d v, d \theta)$, with the same measure defining the $X_{r}(t)^{\prime}$ s in (A.30), it is enough to show that

$$
\begin{aligned}
& \mathbb{E}\left\|X_{r}(t)-\breve{Y}(t)\right\|^{2} \\
& \leq \int_{0}^{\infty} \int_{\mathbb{S}}\left|\widehat{\lambda}_{t}(v \theta)\right|^{2}|\cdot|(1 \wedge(v / r))^{k+1}-\left.1\right|^{2} \cdot\left\|v^{-\mathcal{H}-1 / 2} \mathcal{A}(\theta)\right\|_{\mathrm{op}}^{2}\|\mu\|_{\operatorname{tr}}(d \theta) d v \rightarrow 0,
\end{aligned}
$$

as $r \rightarrow 0$. It is easy to see that since $\lambda_{t} \in \Lambda_{k}$, we have $\left|\widehat{\lambda}_{t}(x)\right| \leq C(1 \wedge\|x\|)^{k+1}, \quad x \in \mathbb{R}^{d}$ Therefore, in view of Relations (5.29) and (A.25), the fact that $\left|(1 \wedge(v / r))^{k+1}-1\right| \rightarrow 0, r \rightarrow$ 0 and the Dominated Convergence Theorem imply (A.31), proving the convergence of the finite-dimensional distributions. To complete the proof of (5.31), we will establish the tightness of $\left\{\breve{X}_{r}(\cdot), r \in(0,1)\right\}$. Since the $\breve{X}_{r}$ 's are Gaussian, as argued above (recall (A.26)), by Proposition 2.4 it is enough to show that for all $M>0$, there exist $C>0$ and $\zeta>0$ such that

$$
\sup _{r \in(0,1)} \mathbb{E}\left\|\breve{X}_{r}(s)-\breve{X}_{r}(t)\right\|^{2} \leq C\|s-t\|^{2 \zeta}, \quad \text { for all } s, t \in B(0, M) .
$$

This is because for $k \geq 0$, the tightness condition on $\left\{\breve{X}_{r}\left(s_{0}\right), r \in(0,1)\right\}$ is automatically fulfilled if one takes $s_{0}=t_{1}$ (recall (2.9) and Remark 2.6).

To this end, we begin with some key observations about the measures $\lambda_{t}$. Since the $c_{j}(t)$ 's in (A.28) are fixed polynomials, we have

$$
\begin{aligned}
\left|\widehat{\lambda}_{s}(v \theta)-\widehat{\lambda}_{t}(v \theta)\right|^{2} & \leq\left|e^{\mathrm{i} s} s^{\top} v \theta-e^{\mathrm{i} t} t^{\top} v \theta\right|^{2}+\sum_{j}\left|c_{j}(s)-c_{j}(t)\right|^{2} \\
& \leq C v^{2}\|s-t\|^{2}+C_{M}\|s-t\|^{2} \leq C_{M}\left(1 \vee v^{2}\right)\|s-t\|^{2},
\end{aligned}
$$


for all $v>0$ and $s, t \in B(0, M)$, where the constant $C_{M}$ does not depend on $v, s, t$ and $\theta \in \mathbb{S}$.

On the other hand, in view of (A.28), the $\hat{\lambda}_{t}$ 's are uniformly bounded for $t \in B(0, M)$ and since $\lambda_{t} \in \Lambda_{k}$ they annihilate polynomials of degree up to $k$ and hence $\widehat{\lambda}_{t}(v \theta)=$ $\mathcal{O}\left(v^{k+1}\right)$. This implies that

$$
\sup _{t, s \in B(0, M), \theta \in \mathbb{S}}\left|\widehat{\lambda}_{s}(v \theta)-\widehat{\lambda}_{t}(v \theta)\right|^{2} \leq C_{M}\left(1 \wedge v^{2(k+1)}\right) .
$$

We are now ready to estimate the difference moments. For all $s, t \in B(0, M)$, we have

$$
\begin{aligned}
\mathbb{E}\left\|\breve{X}_{r}(s)-\breve{X}_{r}(t)\right\|^{2} & \leq \int_{0}^{\infty} \int_{\mathbb{S}}\left|\widehat{\lambda}_{s}(v \theta)-\widehat{\lambda}_{t}(v \theta)\right|^{2}\left\|v^{-\mathcal{H}-1 / 2} \mathcal{A}(\theta)\right\|_{\mathrm{op}}^{2}\|\mu\|_{\operatorname{tr}}(d \theta) d v \\
& =: \int_{0}^{\infty} \int_{\mathbb{S}} g_{s, t}(v, \theta)\|\mu\|_{\operatorname{tr}}(d \theta) d v .
\end{aligned}
$$

Observe first that by (A.33) the bound in (A.25) applies and by (5.29), we have

$$
\int_{\mathbb{S}} g_{s, t}(v, \theta)\|\mu\|_{\operatorname{tr}}(d \theta) \leq C\left(v^{2 \delta-1} 1_{(0,1)}(v)+v^{-2 \epsilon-1} 1_{[1, \infty)}(v)\right),
$$

where the latter function is integrable in $v$ over $(0, \infty)$.

The rest of the strategy is as follows. We will consider the integral of $g_{s, t}$ over three regions $(v, \theta) \in(0, \alpha] \times \mathbb{S},(\alpha, 1 / \alpha) \times \mathbb{S}$ and $[1 / \alpha, \infty) \times \mathbb{S}$. We will choose $\alpha=1 \wedge\|s-t\|^{\kappa}$ for some $\kappa>0$, such that each of the three integrals can be dominated by $C \alpha^{\eta}$, for some positive $\eta$. Namely, let

$$
I_{1}(s, t)+I_{2}(s, t)+I_{3}(s, t):=\left(\int_{(0, \alpha] \times \mathbb{S}}+\int_{(\alpha, 1 / \alpha) \times \mathbb{S}}+\int_{[1 / \alpha, \infty) \times \mathbb{S}}\right) g_{s, t}(v, \theta)\|\mu\|_{\operatorname{tr}}(d \theta) d v .
$$

In view of (A.34), we have

$$
I_{1}(s, t) \leq C \int_{0}^{\alpha} v^{2 \delta-1} d v \leq C \alpha^{2 \delta}
$$

and

$$
I_{3}(s, t) \leq C \int_{1 / \alpha}^{\infty} v^{-2 \epsilon-1} d v \leq C \alpha^{2 \epsilon} .
$$

Now, for the middle piece, using the bound in (A.32), we obtain

$$
I_{2}(s, t) \leq C\|s-t\|^{2} \int_{\alpha}^{1 / \alpha}\left(1 \vee v^{2}\right) d v \leq C\|s-t\|^{2} \alpha^{-3} .
$$

Setting $\|s-t\|^{2} \alpha^{-3}=\alpha^{2(\epsilon \wedge \delta)}$, we see that $\alpha=1 \wedge\|s-t\|^{2 /(3+2(\epsilon \wedge \delta))}$ yields the desired bound $\mathbb{E}\left\|\breve{X}_{r}(s)-\breve{X}_{r}(t)\right\|^{2} \leq \sum_{i=1}^{3} I_{i}(s, t) \leq C\|s-t\|^{2 \zeta}$, uniformly in $r \in(0,1)$, where $\zeta=2(\epsilon \wedge \delta) /(3+2(\epsilon \wedge \delta))>0$.

Lemma A.12. (i) There exists a constant $C$ such that

$$
\int_{1}^{\infty}\left|e^{\dot{\mathrm{i}} \Delta u}-1\right|^{2} u^{-1-\epsilon} d u \leq C \begin{cases}|\Delta|^{2} & , \text { if } \epsilon>2 \\ |\Delta|^{2}|\log | \Delta|| & , \text { if } \epsilon=2 \\ |\Delta|^{\epsilon} & , \text { if } 0<\epsilon<2 .\end{cases}
$$

(ii) Also, for any $\gamma>0$, there is a constant $C_{\gamma}>0$, such that $\int_{0}^{1}\left|e^{\mathrm{i} \Delta u}-1\right|^{2} u^{\gamma-1} d u \leq$ $C_{\gamma}|\Delta|^{2}$.

Proof. Noting that $\left|e^{\mathrm{i} \Delta u}-1\right|^{2}=2(1-\cos (\Delta u))=\mathcal{O}\left(\Delta^{2}\right)$, as $\Delta \rightarrow 0$, the claim in part (ii) is immediate. Part (i) follows by straightforward calculus by considering the change of variables $x:=|\Delta| u$. 


\section{A.7 Proofs for Section 5.3}

Proof of Proposition 5.18: Theorem 5.3 applied to $\mathcal{H}:=H \cdot$ I implies the scaling property of $\chi_{k}$ and the disintegration formula (5.32) in part (ii). Part (iii) follows from Proposition 5.8. We need only prove that $0<H \leq k+1$ and the dichotomy claim (i).

Recall the decomposition in (5.4) and observe that both components $Y_{(\chi, 0)}$ and $Y_{(0, \mathcal{Q})}$ are covariance $H$-self-similar. Notice, however, that $Y_{(0, \mathcal{Q})}$ is always either $(k+1)$-selfsimilar or zero. In particular, for all $c>0$,

$$
c^{H} Y_{(0, \mathcal{Q})}(\lambda) \stackrel{d}{=} Y_{(0, \mathcal{Q})}(c \cdot \lambda) \stackrel{d}{=} c^{k+1} Y_{(0, \mathcal{Q})}(\lambda), \quad \lambda \in \Lambda_{k}
$$

This, since $H$ is scalar, implies $H=k+1$ unless $Y_{(0, \mathcal{Q})} \equiv 0$ (recall Lemma A.6).

Thus, the polynomial spectral component $\mathcal{Q}$ is non-trivial only if $H=k+1$. To complete the proof of (i), it remains to show that if $Y_{(\chi, 0)}$ is non-zero, then its self-similarity exponent is in the range $0<H<k+1$. Firstly, note that $Y_{(\chi, 0)}(c \cdot \lambda) \stackrel{d}{=} c^{H} Y_{(\chi, 0)}(\lambda) \rightarrow 0$, in probability, as $c \downarrow 0$, by the continuity of $Y$. This implies that $H>0$.

Now, observe that by the scaling property of $\chi_{k}$, we have, for all $r \in(0,1)$,

$$
\begin{aligned}
r^{-2 H} \chi_{k}((1, \infty) \times \mathbb{S}) & =\chi_{k}((r, \infty) \times \mathbb{S})=\int_{\{r<\|u\|\}} \frac{1}{1 \wedge\|u\|^{2 k+2}} \chi(d u) \\
& =\int_{\{r<\|u\| \leq 1\}} \frac{1}{\|u\|^{2 k+2}} \chi(d u)+\chi(\{\|u\|>1\}) \\
& =r^{-(2 k+2)} \int_{\{r<\|u\| \leq 1\}}\left(\frac{r}{\|u\|}\right)^{2 k+2} \chi(d u)+\chi(\{\|u\|>1\}) .
\end{aligned}
$$

Since $\chi$ is a finite $\mathbb{T}_{+}$-valued measure, however, the last integral vanishes as $r \downarrow 0$, by the Dominated Convergence Theorem. Thus, by multiplying the last expression by $r^{2 k+2}$, we obtain

$$
r^{2(k+1-H)} \chi_{k}((1, \infty) \times \mathbb{S}) \rightarrow 0,
$$

as $r \rightarrow 0$, which means that $H<k+1$, since by the scaling property $\chi_{k}((r, \infty) \times \mathbb{S}) \neq 0$, for any (all) $r>0$. This completes the proof.

Proof of Theorem 5.19: The proof of Part (i) is as follows. Since $H<k+1$, the polynomial $\mathcal{Q}$ in the spectral characteristic of $\mathcal{K}$ is zero and $\chi$ satisfies (5.32) in polar coordinates. Let $\nu \in \Lambda_{2 k+1}$. Using that $\widehat{\nu}(r \theta)=\int_{\mathbb{R}^{d}} e^{i r \theta^{\top} t} \nu(d t)$, Relation (4.13) in polar coordinates becomes

$$
\begin{aligned}
\mathcal{K}(\nu) & =\int_{\mathbb{S}^{d-1}} \int_{0}^{\infty} \int_{\mathbb{R}^{d}} e^{\mathrm{i} r \theta^{\top} t} \nu(d t) r^{-(2 H+1)} d r \sigma(d \theta) \\
& =\int_{\mathbb{S}^{d-1}} \int_{0}^{\infty} \int_{\mathbb{R}^{d}}\left(\left(e^{\mathrm{i} r \theta^{\top} t}-\sum_{j=0}^{\lfloor 2 H\rfloor} \frac{\left(\mathrm{i} r \theta^{\top} t\right)^{j}}{j !}\right) \nu(d t)\right) r^{-(2 H+1)} d r \sigma(d \theta) \\
& =\int_{\mathbb{S}^{d-1}} \int_{\mathbb{R}^{d}} \int_{0}^{\infty}\left(\left(e^{\mathrm{i} r \theta^{\top} t}-\sum_{j=0}^{\lfloor 2 H\rfloor} \frac{\left(\mathrm{i} r \theta^{\top} t\right)^{j}}{j !}\right) r^{-(2 H+1)} d r\right) \nu(d t) \sigma(d \theta)
\end{aligned}
$$

where the second relation follows from the fact that $\nu \in \Lambda_{2 k+1}$ and therefore we could add a polynomial in $t$ of degree $\lfloor 2 H\rfloor \leq 2 k+1$ without changing the integral. We will justify next the interchange of the inner two integrals leading to (A.35) and compute the inner integral, therein. 
Tangent fields, intrinsic stationarity, and self similarity

For all integer $m \geq 0$, one can show that

$$
\left|e^{\dot{\mathrm{i}} z}-\sum_{j=0}^{m} \frac{(\dot{\mathrm{i}} z)^{j}}{j !}\right| \leq \min \left\{\frac{|z|^{m+1}}{(m+1) !}, \frac{2|z|^{m}}{m !}\right\}, \quad z \in \mathbb{R}
$$

(see e.g., page 298 in Resnick, 1999). Thus, for $0 \leq\lfloor 2 H\rfloor<2 H+1<\lfloor 2 H\rfloor+1$ (recall $2 H$ is not integer), we obtain

$$
\int_{0}^{\infty}\left|e^{\mathrm{i} r \theta^{\top} t}-\sum_{j=0}^{\lfloor 2 H\rfloor} \frac{\left(\mathrm{i} r \theta^{\top} t\right)^{j}}{j !}\right| r^{-(2 H+1)} d r<\infty .
$$

This allows us to interchange the order of the two inner integrals in (A.35). Doing so and making the change of variables $z:=r\left|\theta^{\top} t\right|$, we obtain

$$
\begin{aligned}
& \int_{0}^{\infty}\left(e^{\mathrm{i} r \theta^{\top} t}-\sum_{j=0}^{\lfloor 2 H\rfloor} \frac{\left(\mathrm{i} r \theta^{\top} t\right)^{j}}{j !}\right) r^{-(2 H+1)} d r \\
& =\left|\theta^{\top} t\right|^{2 H} \int_{0}^{\infty}\left(e^{\mathrm{i} z \operatorname{sign}\left(\theta^{\top} t\right)}-\sum_{j=0}^{\lfloor 2 H\rfloor} \frac{\left(\dot{\mathrm{z} z} \operatorname{sign}\left(\theta^{\top} t\right)\right)^{j}}{j !}\right) z^{-(2 H+1)} d z \\
& =\left|\theta^{\top} t\right|^{2 H} \int_{0}^{\infty}\left(\cos (z)-\sum_{0 \leq 2 j \leq\lfloor 2 H\rfloor, j \in \mathbb{Z}} \frac{(-1)^{j} z^{2 j}}{2 j !}\right) z^{-(2 H+1)} d z \\
& +\dot{\mathrm{i}} \operatorname{sign}\left(\theta^{\top} t\right)\left|\theta^{\top} t\right|^{2 H} \int_{0}^{\infty}\left(\sin (z)-\sum_{1 \leq 2 j+1 \leq\lfloor 2 H\rfloor, j \in \mathbb{Z}} \frac{(-1)^{j} z^{2 j+1}}{(2 j+1) !}\right) z^{-(2 H+1)} d z \\
& =\left|\theta^{\top} t\right|^{2 H} I(H)+\dot{\mathrm{i}}\left(\theta^{\top} t\right)^{<2 H>} J(H),
\end{aligned}
$$

where $I(H)$ and $J(H)$ are in (5.34). Note that this argument also demonstrates that $I(H)$ and $J(H)$ are well-defined.

By substituting the last expression in the right-hand side of (A.35), we obtain

$$
\begin{aligned}
\mathcal{K}(\nu)= & I(H) \int_{\mathbb{S}^{d-1}} \int_{\mathbb{R}^{d}}\left|\theta^{\top} t\right|^{2 H} \nu(d t) \sigma(d \theta) \\
& +\dot{\mathrm{i}} J(H) \int_{\mathbb{S}^{d-1}} \int_{\mathbb{R}^{d}}\left(\theta^{\top} t\right)^{<2 H>} \nu(d t) \sigma(d \theta) \\
= & I(H) \int_{\mathbb{S}^{d-1}}|(\theta, \cdot)|^{2 H}(\nu) \sigma(d t)+\dot{\mathrm{i}} J(H) \int_{\mathbb{S}^{d-1}}(\theta, \cdot)^{<2 H>}(\nu) \sigma(d t) .
\end{aligned}
$$

This completes the proof of (5.33).

Part (ii): Suppose now that $2 H \in \mathbb{Z}$, where $1 \leq 2 H \leq 2 k+1$ and observe that $2 H$ may be either even or odd. Using the fact that $\nu \in \Lambda_{2 k+1}$, we get

$$
\begin{aligned}
\widehat{\nu}(r \theta) & =\int_{\mathbb{R}^{d}}\left(e^{\mathrm{i} r \theta^{\top} t}-\sum_{j=0}^{2 H-1} \frac{\left(\mathrm{i} r \theta^{\top} t\right)^{j}}{j !}-1_{[0,1]}(r) \frac{\left(\mathrm{i} r \theta^{\top} t\right)^{2 H}}{(2 H) !}\right) \nu(d t) \\
& =: \int_{\mathbb{R}^{d}} f\left(r \theta^{\top} t, r\right) \nu(d t) .
\end{aligned}
$$

Relation (A.36) implies that $\left|f\left(r \theta^{\top} t, r\right)\right|=\mathcal{O}\left(|r|^{2 H+1}\right)$ as $r \rightarrow 0$ and because of the presence of the indicator function $1_{[0,1]}(r)$, we have $\left|f\left(r \theta^{\top} t, r\right)\right|=\mathcal{O}\left(|r|^{2 H-1}\right)$ as $r \rightarrow \infty$. Therefore,

$$
\int_{0}^{\infty}\left|f\left(r \theta^{\top} t, r\right)\right| r^{-(2 H+1)} d r<\infty,
$$


and by Fubini, we obtain

$$
\begin{aligned}
& \int_{0}^{\infty} \widehat{\nu}(r \theta) r^{-(2 H+1)} d r=\int_{\mathbb{R}^{d}} \int_{0}^{\infty} f\left(r \theta^{\top} t, r\right) r^{-(2 H+1)} d r \nu(d t) \\
& =\int_{\mathbb{R}^{d}}\left[\left|\theta^{\top} t\right|^{2 H} \int_{0}^{\infty}\left(e^{\mathrm{i} z \kappa}-\sum_{j=0}^{2 H-1} \frac{(\dot{\mathrm{i}} z \kappa)^{j}}{j !}-1_{[0,1]}\left(z /\left|\theta^{\top} t\right|\right) \frac{(\dot{\mathrm{i}} z \kappa)^{2 H}}{(2 H) !}\right) z^{-(2 H+1)} d z\right] \nu(d t) \\
& =: \int_{\mathbb{R}^{d}} I_{H}(t) \nu(d t)
\end{aligned}
$$

where we made the change of variables $z:=r\left|\theta^{\top} t\right|$ and where $\kappa:=\operatorname{sign}\left(\theta^{\top} t\right)$.

We now consider two cases. Suppose fist that $2 H$ is even. Upon separating the real and imaginary parts the integral in the r.h.s. of (A.37) becomes:

$$
\begin{aligned}
I_{H}(t) & =\left|\theta^{\top} t\right|^{2 H} \int_{0}^{\infty}\left(\cos (z)-\sum_{\substack{0 \leq 2 j \leq 2 H-1 \\
j \in \mathbb{Z}}} \frac{(-1)^{j} z^{2 j}}{(2 j) !}-1_{[0,1]}\left(z /\left|\theta^{\top} t\right|\right) \frac{(-1)^{H} z^{2 H}}{(2 H) !}\right) z^{-(2 H+1)} d z \\
& +\dot{\mathrm{i}} \kappa\left|\theta^{\top} t\right|^{2 H} \int_{0}^{\infty}\left(\sin (z)-\sum_{\substack{1 \leq 2 j+1 \leq 2 H-1, j \in \mathbb{Z}\\
}} \frac{(-1)^{j} z^{2 j+1}}{(2 j+1) !}\right) z^{-(2 H+1)} d z \\
& =:\left(\theta^{\top} t\right)^{2 H} I_{H, \cos }(t)+\dot{\mathrm{i}}\left(\theta^{\top} t\right)^{<2 H>} J(H) .
\end{aligned}
$$

Observe that the integral in the imaginary part above equals $J(H)$ in (5.34) and where in the real part we dropped the absolute value around $\theta^{\top} t$ since $2 H$ is even.

Since $\left(\theta^{\top} t\right)^{2 H}$ is a polynomial in $t$ of degree $2 H<2 k+1$, we have $\int_{\mathbb{R}^{d}}\left(\theta^{\top} t\right)^{2 H} \nu(d t)=0$. Therefore,

$$
\int_{\mathbb{R}^{d}}\left(\theta^{\top} t\right)^{2 H} I_{H, \cos }(t) \nu(d t)=\int_{\mathbb{R}^{d}}\left(\theta^{\top} t\right)^{2 H}\left(I_{H, \cos }(t)-\mathcal{C}_{H, \cos }\right) \nu(d t),
$$

where

$$
\mathcal{C}_{H, \cos }:=\int_{0}^{\infty}\left(\cos (z)-\sum_{0 \leq 2 j \leq 2 H-1, j \in \mathbb{Z}} \frac{(-1)^{j} z^{2 j}}{(2 j) !}-1_{[0,1]}(z) \frac{(-1)^{H} z^{2 H}}{(2 H) !}\right) z^{-(2 H+1)} d z,
$$

which is almost the same as the first integral in (A.38) except that the indicator function $1_{[0,1]}(z)$ no longer depends on $t$. Notice again that $\mathcal{C}_{H, \text { cos }}$ is well-defined. We thus obtain

$$
\begin{aligned}
I_{H, \cos }(t)-\mathcal{C}_{H, \cos } & =\int_{0}^{\infty}\left(1_{[0,1]}(z)-1_{[0,|\langle\theta, t\rangle|]}(z)\right) \frac{(-1)^{H} z^{2 H}}{(2 H) !} z^{-(2 H+1)} d z \\
& =\frac{(-1)^{H}}{(2 H) !} \int_{\left|\theta^{\top} t\right|}^{1} z^{-1} d z=\frac{(-1)^{H+1}}{(2 H) !} \log \left(\left|\theta^{\top} t\right|\right) .
\end{aligned}
$$

In view of (A.38), (A.39), and (A.40), we get

$$
\begin{aligned}
\mathcal{K}(\nu)= & \int_{\mathbb{S}^{d-1}} \int_{\mathbb{R}^{d}} I_{H}(t) \nu(d t) \sigma(d \theta) \\
= & \frac{(-1)^{H+1}}{(2 H) !} \int_{\mathbb{S}^{d-1}} \int_{\mathbb{R}^{d}}\left(\theta^{\top} t\right)^{2 H} \log \left|\theta^{\top} t\right| \nu(d t) \sigma(d \theta) \\
& \quad+\dot{\mathrm{i}} J(H) \int_{\mathbb{S}^{d-1}} \int_{\mathbb{R}^{d}}\left(\theta^{\top} t\right)^{<2 H>} \nu(d t) \sigma(d \theta) \\
= & \frac{(-1)^{H+1}}{(2 H) !} \int_{\mathbb{S}^{d-1}}\left[(\theta, \cdot)^{2 H} \log |(\theta, \cdot)|\right](\nu) \sigma(d \theta)+\dot{\mathbb{1}} J(H) \int_{\mathbb{S}^{d-1}}(\theta, \cdot)^{<2 H>}(\nu) \sigma(d \theta),
\end{aligned}
$$


which completes the expression in the case when $2 H$ is an even integer.

Suppose now that $2 \mathrm{H}$ is odd. With a very similar argument, using the fact that $\nu \in \Lambda_{2 k+1}$ annihilates polynomials of degree $2 k+1 \geq 2 H$, we obtain that the right-hand side of (A.37) equals

$$
\begin{array}{r}
\int_{\mathbb{R}^{d}}\left|\theta^{\top} t\right|^{2 H} I(H) \nu(d t)+\dot{\mathbb{1}} \int_{\mathbb{R}^{d}} \kappa\left|\theta^{\top} t\right|^{2 H} \int_{0}^{\infty}\left(\sin (z)-\sum_{1 \leq 2 j+1 \leq 2 H-1} \frac{(-1)^{j} z^{2 j+1}}{(2 j+1) !}\right. \\
\left.-1_{[0,1]}\left(z /\left|\theta^{\top} t\right|\right) \frac{(-1)^{H-1 / 2} z^{2 H}}{(2 H) !}\right) z^{-(2 H+1)} d z \nu(d t) \\
=I(H)|(\theta, \cdot)|^{2 H}(\nu)+\dot{\mathbb{1}} \int_{0}^{\infty}\left[\int_{\mathbb{R}^{d}}\left(\theta^{\top} t\right)^{2 H}\left(1_{[0,1]}(z)-1_{\left[0,1 /\left|\theta^{\top} t\right|\right]}(z)\right) \frac{(-1)^{H-1 / 2} z^{-1}}{(2 H) !} \nu(d t)\right] d z,
\end{array}
$$

where we used the fact that $\kappa\left|\theta^{\top} t\right|^{2 H}=\left(\theta^{\top} t\right)^{2 H}$ and the last relation is obtained with the same strategy as in (A.39). More precisely, applying Fubini and using that $\int_{\mathbb{R}^{d}}\left(\theta^{\top} t\right)^{2 H} \nu(d t)=0$, allows us to eliminate the terms involving $\sin (z)$ and $z^{2 j+1}$. At the same time, we add the term $\left(\theta^{\top} t\right)^{2 H} 1_{[0,1]}(z) z^{-1}$, which is a polynomial in $t$ also annihilated by $\nu$.

Now, since the inner integrand in the r.h.s. of (A.41) is integrable with respect to $z$, another application of Fubini shows that right-hand side of (A.37) equals

$$
\begin{aligned}
& I(H)|(\theta, \cdot)|^{2 H}(\nu)+\frac{\dot{\mathbb{1}}(-1)^{H-1 / 2}}{(2 H) !} \int_{\mathbb{R}^{d}}\left(\theta^{\top} t\right)^{2 H}\left[\int_{1 /\left|\theta^{\top} t\right|}^{1} z^{-1} d z\right] \nu(d t) \\
& =I(H)|(\theta, \cdot)|^{2 H}(\nu)+\frac{\dot{\mathbb{i}}(-1)^{H+1 / 2}}{(2 H) !} \int_{\mathbb{R}^{d}}\left(\theta^{\top} t\right)^{2 H} \log \left(\left|\theta^{\top} t\right|\right) \nu(d t) .
\end{aligned}
$$

This leads to the desired expression

$$
\mathcal{K}(\nu)=I(H) \int_{\mathbb{S}^{d-1}}|(\theta, \cdot)|^{2 H}(\nu) \sigma(d \theta)+\dot{\mathbb{i}} \frac{(-1)^{H+1 / 2}}{(2 H) !} \int_{\mathbb{S}^{d-1}}\left[(\theta, \cdot)^{2 H} \log |(\theta, \cdot)|\right](\nu) \sigma(d \theta),
$$

completing the proof.

\section{References}

P. Abry and G. Didier. Wavelet estimation for operator fractional Brownian motion. Bernoulli, 24(2):895-928, 2018. MR3706780

P. Abry, H. Wendt, S. Jaffard, and G. Didier. Multivariate scale-free temporal dynamics: From spectral (Fourier) to fractal (wavelet) analysis. Comptes Rendus Physique, 20(5):489 - 501, 2019.

P. Amblard and J. Coeurjolly. Identification of the multivariate fractional Brownian motion. IEEE Transactions on Signal Processing, 59(11):5152-5168, 2011. doi: 10.1109/TSP.2011.2162835. MR2883588

C. Baek, G. Didier, and V. Pipiras. On integral representations of operator fractional Brownian fields. Statist. Probab. Lett., 92:190-198, 2014. ISSN 0167-7152. doi: 10.1016/j.spl.2014.05.015. URL https://doi.org/10.1016/j.spl.2014.05.015. MR3230493

G. Berschneider. Spectral representation of intrinsically stationary fields. Stochastic Process. Appl., 122(12):3837-3851, 2012. ISSN 0304-4149. doi: 10.1016/j.spa.2012.07.005. URL https: //doi.org/10.1016/j.spa.2012.07.005. MR2971716

G. Berschneider and Z. Sasvári. Spectral theory of stationary random fields and their generalizations. A short historical survey. In Indefinite Inner Product Spaces, Schur analysis, and Differential Equations, volume 263 of Oper. Theory Adv. Appl., pages 217-235. Birkhäuser/Springer, Cham, 2018. MR3728932 
Tangent fields, intrinsic stationarity, and self similarity

H. Biermé and C. Lacaux. Fast and exact synthesis of some operator scaling Gaussian random fields. Appl. Comput. Harmon. Anal., 48(1):293-320, 2020. ISSN 1063-5203. doi: 10.1016/j.acha. 2018.05.004. URL https://doi.org/10.1016/j.acha.2018.05.004. MR4016993

H. Biermé, M. M. Meerschaert, and H.-P. Scheffler. Operator scaling stable random fields. Stochastic Process. Appl., 117(3):312-332, 2007. ISSN 0304-4149. doi: 10.1016/j.spa.2006.07.004. URL https://doi.org/10.1016/j.spa.2006.07.004.

P. Billingsley. Convergence of Probability Measures. Wiley Series in Probability and Statistics: Probability and Statistics. John Wiley \& Sons, Inc., New York, second edition, 1999. ISBN 0-471-19745-9. doi: 10.1002/9780470316962. URL https://doi.org/10.1002/9780470316962. A Wiley-Interscience Publication. MR1700749

S. Bochner. Vorlesungen über Fouriersche integrale. Chelsea Publishing Company, 1948.

J.-P. Chilès and P. Delfiner. Geostatistics. Wiley Series in Probability and Statistics. John Wiley \& Sons, Inc., Hoboken, NJ, second edition, 2012. ISBN 978-0-470-18315-1. URL https://doi.org/10. 1002/9781118136188. Modeling spatial uncertainty. MR2850475

J. B. Conway. A Course in Functional Analysis. Springer, 2007. MR0768926

H. Cramér. On harmonic analysis in certain functional spaces. Ark. Mat. Astr. Fys., 28B(12):7, 1942. ISSN 0004-2080. MR0006609

Y. Davydov and V. Paulauskas. Lamperti type theorems for random fields, 2017. arXiv:1705.00182. MR3833096

G. Didier and V. Pipiras. Integral representations and properties of operator fractional Brownian motions. Bernoulli, 17(1):1-33, 2011. ISSN 1350-7265. doi: 10.3150/10-BEJ259. URL https: //doi.org/10.3150/10-BEJ259. MR2797980

G. Didier, M. M. Meerschaert, and V. Pipiras. Exponents of operator self-similar random fields. J. Math. Anal. Appl., 448(2):1450-1466, 2017. ISSN 0022-247X. doi: 10.1016/j.jmaa.2016.11.055. URL https://doi.org/10.1016/j.jmaa.2016.11.055.

G. Didier, M. M. Meerschaert, and V. Pipiras. Domain and range symmetries of operator fractional Brownian fields. Stochastic Process. Appl., 128(1):39-78, 2018. ISSN 0304-4149. doi: 10.1016/j. spa.2017.04.003. URL https://doi.org/10.1016/j.spa.2017.04.003.

R. L. Dobrushin. Gaussian and their subordinated self-similar random generalized fields. The Annals of Probability, 7:1-28, 1979. MR0515810

R. M. Dudley. Real Analysis and Probability, volume 74 of Cambridge Studies in Advanced Mathematics. Cambridge University Press, Cambridge, 2002. ISBN 0-521-00754-2. doi: 10.1017/ CBO9780511755347. URL https://doi.org/10.1017/CBO9780511755347. Revised reprint of the 1989 original. MR1932358

J. Dugundji. Topology. Allyn and Bacon, Inc., Boston, Mass., 1966. MR0193606

M.-C. Düker. Limit theorems in the context of multivariate long-range dependence. Stochastic Process. Appl., 130(9):5394-5425, 2020. ISSN 0304-4149. doi: 10.1016/j.spa.2020.03.011. URL https://doi.org/10.1016/j.spa.2020.03.011. MR4127333

A. Durand and F. Roueff. Spectral analysis of weakly stationary processes valued in a separable Hilbert space. 2020. arXiv:1910.08491.

K. J. Falconer. The geometry of fractal sets, volume 85 of Cambridge Tracts in Mathematics. Cambridge University Press, Cambridge, 1986. ISBN 0-521-25694-1; 0-521-33705-4. MR0867284

K. J. Falconer. Tangent fields and the local structure of random fields. J. Theoret. Probab., 15(3):731750, 2002. ISSN 0894-9840. doi: 10.1023/A:1016276016983. URL https://doi.org/10.1023/A: 1016276016983.

K. J. Falconer. The local structure of random processes. J. London Math. Soc. (2), 67(3):657672, 2003. ISSN 0024-6107. doi: 10.1112/S0024610703004186. URL https://doi.org/10.1112/ S0024610703004186.

I. M. Gel'fand and N. Y. Vilenkin. Generalized Functions. Vol. 4: Applications of harmonic analysis. Translated by Amiel Feinstein. Academic Press, New York - London, 1964, 1964. MR0173945

B. Gnedenko. Sur la distribution limite du terme maximum d'une série aléatoire. Ann. of Math. (2), 44:423-453, 1943. ISSN 0003-486X. MR0008655 
Tangent fields, intrinsic stationarity, and self similarity

I. Gohberg, S. Goldberg, and N. Krupnik. Traces and Determinants of Linear Operators, volume 116 of Operator Theory: Advances and Applications. Birkhäuser Verlag, Basel, 2000. ISBN 37643-6177-8. doi: 10.1007/978-3-0348-8401-3. URL https://doi.org/10.1007/978-3-0348-8401-3. MR1744872

R. B. Holmes. Mathematical foundations of signal processing. SIAM Review, 21(3):361-388, 1979. MR0535119

L. Horváth and P. Kokoszka. Inference for functional data with applications. Springer Series in Statistics. Springer, New York, 2012. ISBN 978-1-4614-3654-6. doi: 10.1007/978-1-4614-3655-3. URL https://doi.org/10.1007/978-1-4614-3655-3. MR2920735

T. Hsing and R. Eubank. Theoretical Foundations of Functional Data Analysis, with an Introduction to Linear Operators. Wiley Series in Probability and Statistics. John Wiley \& Sons, Ltd., Chichester, 2015. ISBN 978-0-470-01691-6. doi: 10.1002/9781118762547. URL https://doi.org/10.1002/9781118762547.

H. Hult and F. Lindskog. Regular variation for measures on metric spaces. Publ. Inst. Math. (Beograd) (N.S.), 80(94):121-140, 2006. ISSN 0350-1302. doi: 10.2298/PIM0694121H. URL http://dx.doi.org/10.2298/PIM0694121H.

Z. J. Jurek. Polar coordinates in Banach spaces. Bull. Polish Acad. Sci. Math., 32(1-2):61-66, 1984. ISSN 0239-7269. MR0766981

O. Kallenberg. Foundations of Modern Probability. Probability and its Applications (New York). Springer-Verlag, 1997. MR1464694

G. Kallianpur and V. Mandrekar. Spectral theory of stationary H-valued processes. Journal of Multivariate Analysis, 1(1):1-16, 1971. MR0301788

S. Kechagias and V. Pipiras. Definitions and representations of multivariate long-range dependent time series. J. Time Series Anal., 36(1):1-25, 2015. ISSN 0143-9782. doi: 10.1111/jtsa.12086. URL https://doi.org/10.1111/jtsa.12086. MR3300202

A. Khintchine. Korrelationstheorie der stationären stochastischen prozesse. Mathematische Annalen, 109(1):604-615, 1934. MR1512911

M. Kuczma. An introduction to the Theory of Functional Equations and Inequalities. Birkhäuser Verlag, Basel, second edition, 2009. ISBN 978-3-7643-8748-8. doi: 10.1007/978-3-7643-8749-5. URL https://doi.org/10.1007/978-3-7643-8749-5. Cauchy's equation and Jensen's inequality, Edited and with a preface by Attila Gilányi. MR2467621

K. Kuratowski. Introduction to Set Theory and Topology. PWN-Polish Scientific Publishers, Warsaw; Pergamon Press, Oxford-New York-Toronto, Ont., 1977. Containing a supplement, "Elements of algebraic topology" by Ryszard Engelking, Translated from the Polish by Leo F. Boroń, International Series of Monographs in Pure and Applied Mathematics, 101. MR0643829

J. Lamperti. Semi-stable stochastic processes. Trans. Amer. Math. Soc., 104:62-78, 1962. ISSN 0002-9947. doi: 10.2307/1993933. URL https://doi.org/10.2307/1993933.

Y. Li and Y. Xiao. Multivariate operator-self-similar random fields. Stochastic Process. Appl., 121(6):1178-1200, 2011. ISSN 0304-4149. doi: 10.1016/j.spa.2011.02.005. URL https://doi.org/ 10.1016/j.spa.2011.02.005. MR2794972

P. A. Loeb and E. Talvila. Lusin's theorem and Bochner integration. Sci. Math. Jpn., 60(1):113-120, 2004. ISSN 1346-0862. MR2072104

J. D. Mason and Y. Xiao. Sample path properties of operator-self-similar Gaussian random fields. Teor. Veroyatnost. i Primenen., 46(1):94-116, 2001. ISSN 0040-361X. doi: 10.1137/ S0040585X97978749. URL https://doi.org/10.1137/S0040585X97978749.

G. Matheron. The intrinsic random functions and their applications. Advances in Appl. Probability, 5:439-468, 1973. ISSN 0001-8678. doi: 10.2307/1425829. URL https://doi.org/10.2307/1425829.

M. M. Meerschaert. Multivariate Domains of Attraction and Regular Variation. PhD thesis, University of Michigan, Ann Arbor, 1984. MR2633908

M. M. Meerschaert and H.-P. Scheffler. Limit distributions for sums of independent random vectors. Wiley Series in Probability and Statistics: Probability and Statistics. John Wiley \& Sons Inc., New York, 2001. ISBN 0-471-35629-8. Heavy tails in theory and practice. MR1840531 
Tangent fields, intrinsic stationarity, and self similarity

J. R. Munkres. Topology. Prentice Hall, Inc., Upper Saddle River, NJ, 2000. ISBN 0-13-181629-2. Second edition of [MR0464128]. MR3728284

G. J. Murphy. Positive definite kernels and Hilbert $C^{*}$-modules. Proc. Edinburgh Math. Soc. (2), 40(2):367-374, 1997. ISSN 0013-0915. doi: 10.1017/S0013091500023804. URL https: //doi.org/10.1017/S0013091500023804.

K.-H. Neeb. Operator-valued positive definite kernels on tubes. Monatsh. Math., 126(2):125-160, 1998. ISSN 0026-9255. doi: 10.1007/BF01473583. URL https://doi.org/10.1007/BF01473583.

J.-P. Pellonpää and K. Ylinen. Modules, completely positive maps, and a generalized KSGNS construction. Positivity, 15(3):509-525, 2011. ISSN 1385-1292. doi: 10.1007/s11117-010-0104-6. URL https://doi.org/10.1007/s11117-010-0104-6. MR2832603

E. Perrin, R. Harba, C. Berzin-Joseph, I. Iribarren, and A. Bonami. nth-order fractional Brownian motion and fractional Gaussian noises. IEEE Transactions on Signal Processing, 49(5):10491059, 2001.

D. Preiss. Geometry of measures in $\mathbf{R}^{n}$ : distribution, rectifiability, and densities. Ann. of Math. (2), 125(3):537-643, 1987. ISSN 0003-486X. doi: 10.2307/1971410. URL https://doi.org/10.2307/ 1971410 .

J. O. Ramsay and B. W. Silverman. Functional data analysis. Springer Series in Statistics. Springer, New York, second edition, 2005. ISBN 978-0387-40080-8; 0-387-40080-X. MR2168993

S. I. Resnick. A Probability Path. Birkhäuser Boston Inc., Boston, MA, 1999. ISBN 0-8176-4055-X. MR1664717

S. Rolewicz. Metric Linear Spaces, volume 20 of Mathematics and its Applications (East European Series). D. Reidel Publishing Co., Dordrecht; PWN-Polish Scientific Publishers, Warsaw, second edition, 1985. ISBN 90-277-1480-0. MR0808176

Z. Sasvári. Correlation functions of intrinsically stationary random fields. In Modern Analysis and Applications. The Mark Krein Centenary Conference. Vol. 1: Operator theory and related topics, volume 190 of Oper. Theory Adv. Appl., pages 451-470. Birkhäuser Verlag, Basel, 2009. doi: 10.1007/978-3-7643-9919-1_28. URL https://doi.org/10.1007/978-3-7643-9919-1 28. MR2568647

J. Shen. Local Structure of Random Fields - Properties and Inference. ProQuest LLC, Ann Arbor, MI, 2019. ISBN 978-1687-99522-3. URL http://gateway.proquest.com/openurl?url_ver=Z39. 88-2004\&rft_val_fmt=info:ofi/fmt:kev:mtx:dissertation\&res_dat=xri:pqm\&rft_dat=xri:pqdiss: 27614372. Thesis (Ph.D.)-University of Michigan.

J. Shen, S. Stoev, and T. Hsing. Tangent fields, intrinsic stationarity, and self similarity (with a supplement on Matheron Theory), 2020. URL https://arxiv.org/abs/2010.14715.

B. Simon. A comprehensive Course in Analysis. American Mathematical Society Providence, Rhode Island, 2015. MR3443339

M. L. Stein. Interpolation of Spatial Data. Springer Series in Statistics. Springer-Verlag, New York, 1999. ISBN 0-387-98629-4. doi: 10.1007/978-1-4612-1494-6. URL https://doi.org/10.1007/ 978-1-4612-1494-6. Some theory for Kriging. MR1697409

A. van Delft and M. Eichler. A note on Herglotz's theorem for time series on function spaces. Stochastic Process. Appl., 130(6):3687-3710, 2020. MR4092417

Acknowledgments. We dedicate this paper to the memory of Mark Marvin Meerschaert (1955-2020). Mark was a great visionary, mentor, and friend to us. His work has inspired and guided us in this paper and elsewhere. We are very grateful to Rafail Kartsioukas for his exceptionally careful reading of the manuscript and help with fixing a number of important mathematical errors. We are also indebted to two anonymous referees for their exceptionally detailed and insightful reports. They helped us correct a number of errors and improve the mathematical rigor, clarity, and substance of our results. 